\title{
The utility of physiotherapy assessments delivered by telehealth: A systematic review
}

Cherie Zischke ${ }^{1,2}$, Vinicius

Simas ${ }^{1}$, Wayne Hing ${ }^{1}$,

Nikki Milne ${ }^{1}$, Alicia

Spittle $^{3}$, Rodney Pope ${ }^{4}$

${ }^{1}$ Faculty of Health Sciences and Medicine, Bond Institute of Health and Sport, Bond University, Robina, Queensland, Australia

${ }^{2}$ School of Allied Health, Exercise and Sports Sciences, Charles Sturt University, Port Macquarie, New South Wales, Australia ${ }^{3}$ Faculty of Medicine, Dentistry and Health Sciences, The University of Melbourne, Melbourne, Victoria, Australia ${ }^{4}$ School of Allied Health, Exercise and Sports Sciences, Charles Sturt University, Albury, New South Wales, Australia

\section{Correspondence to:}

Cherie Zischke (BExSc, MPhysio) Faculty of Science and Health School of Allied Health, Exercise and Sports Sciences

Charles Sturt University

7 Major Innes Rd

Port Macquarie NSW 2444

Australia

czischke@csu.edu.au
Background Telehealth use is increasing due to its ability to overcome service access barriers and provide continued care when disease transmission is of concern. However, little is known of the validity, reliability and utility of performing physiotherapy assessments using synchronous forms of telehealth across all physiotherapy practice areas. The aim of this systematic review was to determine the current clinometric value of performing physiotherapy assessments using synchronous forms of telehealth across all areas of physiotherapy practice.

Methods A comprehensive search of databases (PubMed/MEDLINE, The Cochrane Library, Embase and EBSCO) was undertaken to identify studies investigating the clinometric value of performing physiotherapy assessments using synchronous forms of telehealth across all physiotherapy practice areas. Following selection, a quality appraisal was conducted using the Brink and Louw or Mixed Methods Appraisal Tool. Evidence regarding validity, reliability and utility of synchronous telehealth physiotherapy assessments was extracted and synthesised using a critical narrative approach.

Results Thirty-nine studies conducted in a variety of simulated ( $\mathrm{n}=15$ ) or real-world telehealth environments $(n=24)$, were included. The quality of the validity, reliability and utility studies varied. Assessments including range of movement, muscle strength, endurance, pain, special orthopaedic tests (shoulder/elbow), Berg Balance Scale, timed up and go, timed stance test, six-minute walk test, steps in 360-degree turn, Movement Assessment Battery for Children ( $2^{\text {nd }}$ Edition), step test, ABILHAND assessment, active straight leg raise, and circumferential measures of the upper limb were reported as valid/reliable in limited populations and settings (many with small sample sizes). Participants appeared to embrace telehealth technology use, with most studies reporting high levels of participant satisfaction. If given a choice, many reported a preference for in-person physiotherapy assessments. Some inconsistencies in visual/auditory quality and challenges with verbal/non-verbal communication methods were reported. Telehealth was considered relatively cost-effective once services were established.

Conclusions Performing physiotherapy assessments using synchronous forms of telehealth appears valid and reliable for specific assessment types in limited populations. Further research is needed in all areas of physiotherapy practice, to strengthen the evidence surrounding its clinometric value. Clinicians contemplating using this assessment mode should consider the client/family preferences, assessment requirements, cultural needs, environment, cost considerations, access and confidence using technology.

Protocol registration PROSPERO: CRD42018108166. 
Reduced or delayed access to health care services can have detrimental effects on the health and well-being of individuals [1]. Those living in regional, rural and remote areas with a lower population density are more likely to encounter reduced access to health care services [2], including access to physiotherapy services in both the public and private sectors $[3,4]$. This access limitation is particularly pronounced for more specialised areas of physiotherapy, such as those that address disability and paediatrics [5].

The use of telehealth services to improve physiotherapy access is increasing due to its ability to overcome the barriers of distance [6], service provider availability [7] and capacity to provide safe care to community members during periods of time when disease transmission is of concern. Telehealth has been defined by the World Health Organisation (WHO) as 'the use of telecommunications and virtual technology to deliver health care outside of traditional health care facilities' [8]. The information and communication technologies (ICTs) used in the delivery of telehealth services allow the transmission of health information such as voice, data, and still or video images over short and long distances, without the need for recipients of care or health professionals to be co-located [9]. Telehealth includes delivery of assessment and diagnosis, treatment, and preventative and curative aspects of health care [9], and can be provided using synchronous methods (real-time), or asynchronous methods (store and forward) [10].

Physiotherapy consultations performed using synchronous forms of telehealth have become even more common in recent times, due to the COVID-19 pandemic. Maintaining access to physiotherapy services during this time has been challenging for many health care providers $[11,12]$ where area lockdowns and distancing requirements have interrupted and limited face-to-face service provision [11], initiating a shift in the way physiotherapy services are provided to clients [13-15]. Many health services and/or professional associations, such as the Australian Physiotherapy Association, NHS and Chartered Society of Physiotherapy (UK), have released advice and guidelines to support clinicians providing telehealth services during the COVID-19 pandemic $[16,17]$. Although this increased use of telehealth during the COVID-19 pandemic has been essential in ensuring continuity of care from many allied health and medical practitioners $[14,18]$, concerns have been raised regarding the limited evidence supporting these changes to physiotherapy practice across the lifespan $[13,15]$.

A physiotherapy client assessment, performed at commencement of physiotherapy service delivery and at regular intervals throughout the stages of intervention, provides vital information to therapists about the need for, and response to, therapeutic interventions [19]. Such assessment is essential for treatment plan development, clinical reasoning processes, intervention selection, and implementation of effective treatment strategies with clients [20]. Where a physiotherapist is not available to provide a face-to-face assessment, assessment via telehealth may need to be considered.

A variety of studies have investigated the use of telehealth to perform physiotherapy assessment and interventions with adult populations [21-23] however, performing accurate physiotherapy assessments using this method can be challenging [24]. Two systematic reviews, both limited to the field of musculoskeletal (MSK) physiotherapy and with a focus on the adult population, have been conducted $[19,25]$ to investigate the validity and reliability of conducting physiotherapy assessment via telehealth. In 2017, Mani and colleagues investigated the use of internet-based physiotherapy assessments within the MSK field of physiotherapy, concluding that it is feasible to use telehealth systems to assess swelling, range of movement, pain, muscle strength, balance, gait and functional outcomes in adults referred to physiotherapy [19]. In 2018, Grona and colleagues investigated the validity and reliability of synchronous videoconference use in the assessment and management of adults presenting with MSK conditions [25]. Grona and colleagues concluded that synchronous video-based telehealth systems could be viable when assessing and managing adults with MSK conditions but may have poor reliability for assessments of the shoulder joint and elbow joint, and for nerve tests, scar assessment and lumbar posture assessment [25]. A small number of systematic reviews on telehealth have been conducted in other settings such as neurology [26,27] and aged care [28], with a focus on health and activity, using asynchronous monitoring systems or wearable devices.

To enhance physiotherapy assessment and care, it is important to understand the reliability, validity and utility of performing synchronous telehealth assessments throughout all areas of physiotherapy practice, across the lifespan. To our knowledge, there has not been a recent review of the literature investigating this topic. Therefore, the aim of this systematic review was to determine the current clinometric value (reliability, validity and broader utility) of physiotherapy assessments delivered using synchronous forms of telehealth, considering the full breadth of fields of physiotherapy practice. A second aim was to identify which of the validity, reliability and utility studies have been conducted in real-world rural or remote contexts and to provide a summary of their findings. 
Table 1. Literature database search strategy

\begin{tabular}{|c|c|}
\hline DATABASES & SEARCH TERMS \\
\hline PubMed/MEDLINE & $\begin{array}{l}\text { Physiotherapist; OR physical therapist; } \\
\text { OR physiotherapy; OR physical }\end{array}$ \\
\hline The Cochrane Library & therapy; OR rehabilitation \\
\hline Embase & AND \\
\hline EBSCO (Academic Search Complete, & Telehealth; OR telemedicine; \\
\hline AHFS Consumer Medication & OR telecare; OR e-health; OR \\
\hline Information, CINAHL, Computers & telemental; \\
\hline \& Applied Sciences Complete, & OR telehealth care; OR telemonitoring; \\
\hline $\begin{array}{l}\text { Education Research Complete, } \\
\text { ERIC Health Business Elite Health }\end{array}$ & OR telerehabilitation; OR telepractice \\
\hline $\begin{array}{l}\text { ERIC, Health Business Elite, Health } \\
\text { Source - Consumer edition, }\end{array}$ & AND \\
\hline Health Source: Nursing/Academic & Assess*; OR evaluat*; OR analys*; \\
\hline Edition, MasterFILE Premier and & OR measur*; OR reliability; OR validity; \\
\hline SPORTDiscus with full text) & OR utility. \\
\hline
\end{tabular}

Table 2. Inclusion and exclusion criteria

\begin{tabular}{lll}
$\begin{array}{l}\text { STUDY } \\
\text { CHARACTERISTICS }\end{array}$ & INCLUSION CRITERIA & EXCLUSION CRITERIA \\
& $\begin{array}{l}\text { Validity studies, reliability } \\
\text { studies and studies examining } \\
\text { the broader utility of } \\
\text { performing physiotherapy } \\
\text { assessments conducted } \\
\text { using synchronous forms of } \\
\text { telehealth }\end{array}$ & $\begin{array}{l}\text { Studies of interventions, } \\
\text { or assessments using } \\
\text { asynchronous forms of } \\
\text { telehealth, and those that } \\
\text { contained insufficient } \\
\text { methodological detail of the } \\
\text { telehealth assessment }\end{array}$ \\
\hline $\begin{array}{l}\text { Physiotherapists or } \\
\text { physiotherapy students } \\
\text { (under the supervision of a } \\
\text { physiotherapist }\end{array}$ & $\begin{array}{l}\text { Studies that involved only } \\
\text { health professionals other than } \\
\text { physiotherapists performing } \\
\text { or interpreting assessments } \\
\text { conducted using synchronous } \\
\text { forms of telehealth }\end{array}$ \\
\hline
\end{tabular}

\section{METHODS}

\section{Systematic review}

This systematic review protocol was registered with PROSPERO (CRD42018108166).

\section{Search strategy}

A comprehensive search of key literature databases was undertaken on 17th December 2020 to identify studies that addressed the aims of this review (Table 1). A hand search of reference lists of included studies was conducted following this to identify additional studies for inclusion. Databases were searched for articles published between January 2010 and 17th December 2020 inclusive. This date range was adopted to ensure inclusion of studies using contemporary technologies. The initial search was not restricted by language.

\section{Inclusion and exclusion criteria}

The inclusion and exclusion criteria used for the selection of studies in this review are listed in Table 2. Authors of studies that did not specify the discipline of the health professional who conducted the telehealth assessment were contacted to ascertain this detail.

\section{Study selection}

Screening and study selection commenced following completion of the search process. References of articles identified in the search were imported into EndNote (Clarivate, Philadelphia, PA, USA) [29] and duplicates removed. Titles and abstracts were screened, with articles that were clearly ineligible excluded. Full texts of remaining articles were sourced and reviewed by two independent reviewers ( $\mathrm{CZ}$ and RP) to determine final eligibility based on the inclusion and exclusion criteria, with reasons for exclusion noted. Any discrepancies were resolved through discussion and consensus, with a third reviewer (WH) available to be consulted if consensus could not be reached. Studies deemed eligible for inclusion in this review were retained and analysed. The results of the search, screening and selection process were recorded in a PRISMA flow diagram (Preferred Reporting Items for Systematic Reviews) [30]. The PRISMA Checklist [30] was completed and can be found in Table S1 in the Online Supplementary Document.

\section{Quality assessment}

The methodological quality of validity and reliability studies included in this review was assessed by two independent reviewers (CZ and VS) using the Brink and Louw critical appraisal tool (CAT) [31]. A third researcher (RP) was available to be consulted regarding any discrepancies that could not be resolved by discussion or consensus. The Brink and Louw CAT was developed in 2011 and was based on the Quality Appraisal of Diagnostic Reliability Studies (QAREL) and Quality Assessment of Diagnostic Accuracy Studies (QUADAS) tools $[31,32]$. The CAT was designed for use in systematic reviews that investigate validity and reliability of assessment tools. It uses a series of 13 questions scored as 'yes', 'no' or 'not applicable', with five of the items referring to validity and reliability, four items specific to validity and the remaining four items specific to reliability [31]. The CAT does not provide a quality rating [32]. To provide a quality rating for each study, we calculated a quality percentage and subsequent rating (Table 3) based on the number of items where 'yes' was given 
Table 3. Quality ratings for methodological quality assessment

\begin{tabular}{|c|c|c|c|c|}
\hline \multicolumn{5}{|c|}{ QUALITY RATING (SCORE RANGE \%) } \\
\hline $\begin{array}{l}\text { Very poor } \\
(0 \%-19 \%)\end{array}$ & $\begin{array}{c}\text { Poor } \\
(20 \%-39 \%)\end{array}$ & $\begin{array}{c}\text { Moderate } \\
(40 \%-59 \%)\end{array}$ & $\begin{array}{c}\text { Good } \\
(60 \%-79 \%)\end{array}$ & $\begin{array}{c}\text { Very good } \\
(\geq 80 \%)\end{array}$ \\
\hline
\end{tabular}

as the response, divided by the total number of items available to be scored, depending on the study type. Similar rating scales have been used by other authors when employing the Brink and Louw CAT, reporting a score of $\geq 60 \%$ as indicating 'high quality [32-35].

The methodological quality of the utility studies included in this review was similarly assessed by two independent reviewers (CZ and VS), using the Mixed Methods Appraisal Tool (MMAT), version 2018, which is a critical appraisal tool developed by Hong and colleagues [36]. The MMAT is used to appraise the quality of various types of empirical studies [37]. The results are based on ratings for five criteria [37]. An overall rating was determined based on recommendations from Hong [38], who suggested a percentage rating for the number of 'yes' responses could be used if a quality rating score was required. These percentage scores were converted to quality descriptors, equivalent to those used for the Brink and Louw CAT (Table 3). The level of agreement between the two raters in ratings of methodological quality was ascertained by calculation of a Cohen's kappa coefficient $(\mathrm{k})$ [39].

Case studies or case series that were identified as eligible for inclusion in this review were analysed descriptively and did not undergo a separate methodological quality rating, being considered to provide low level (IV) evidence [40].

\section{Data extraction, synthesis and analysis}

Key data and outcomes from included articles relating to the study design and analysis, aims, participants, area of physiotherapy practice and setting, assessment environment, assessment delivery and technology, assessment/outcome measures, and reliability, validity and utility of physiotherapy assessments conducted via synchronous telehealth, were extracted manually by a single reviewer. This data was summarised in a Microsoft Excel (Microsoft Inc, Seattle, WA, USA) spreadsheet to ensure a standard approach, to examine variation and for ease of analysis. Findings were synthesised using a critical narrative approach in which the strength of evidence from individual studies was considered, based on the methodological quality of each study. Findings of individual studies were reported in tables and figures with particular consideration given, in the reporting of results, to findings from studies performed in real-world rural or remote contexts rather than simulated contexts. A meta-analysis was not conducted due to the clinical and methodological diversity in the types and methods of telehealth assessments used and heterogeneity of the reported outcomes of the studies included in the review.

\section{Deviations from the protocol}

Some minor deviations from the original protocol published with PROSPERO (CRD42018108166) were made to improve the rigor of this review. The date range for the original protocol was altered, with search originally to be conducted in August 2018. The search was subsequently completed in December 2020. The inclusion and exclusion criteria were further refined to focus on synchronous forms of telehealth. The MMAT was also included as the critical appraisal tool used to appraise utility studies included in this review. Finally, one further author (VS) who was integral in completing this review was added to the author list.

\section{RESULTS}

The results of the search, screening and selection process can be found in Figure 1. Thirty-nine articles were retained following screening and selection. The third reviewer was not required for the small number of articles where there was initial disagreement regarding eligibility $(n=5)$, as consensus regarding eligibility was reached through discussion between the two primary reviewers.

\section{Study types and methodological quality}

The methodological quality of each included study is summarised in Table 4. There was a strong level of agreement between the two reviewers' scores, $\mathrm{k}=0.90$ (95\% CI, 0.85 to 0.96), $P<0.001$. Of the 39 articles reviewed, 18 studies investigated the concurrent validity and/or reliability of synchronous telehealth physiotherapy assessments (Table 4). The methodological quality of the validity and reliability studies ranged from 'good' ( $\mathrm{n}=10)$ to 'very good' $(\mathrm{n}=8)$. Most studies described participants in detail $(\mathrm{n}=13)$ and the index test in enough detail to enable replication $(n=12)$. The statistical methods used within each article, although varied, were appropriate to the research question. One study failed to explain participant withdrawals and one did not provide qualification details of the raters involved. 


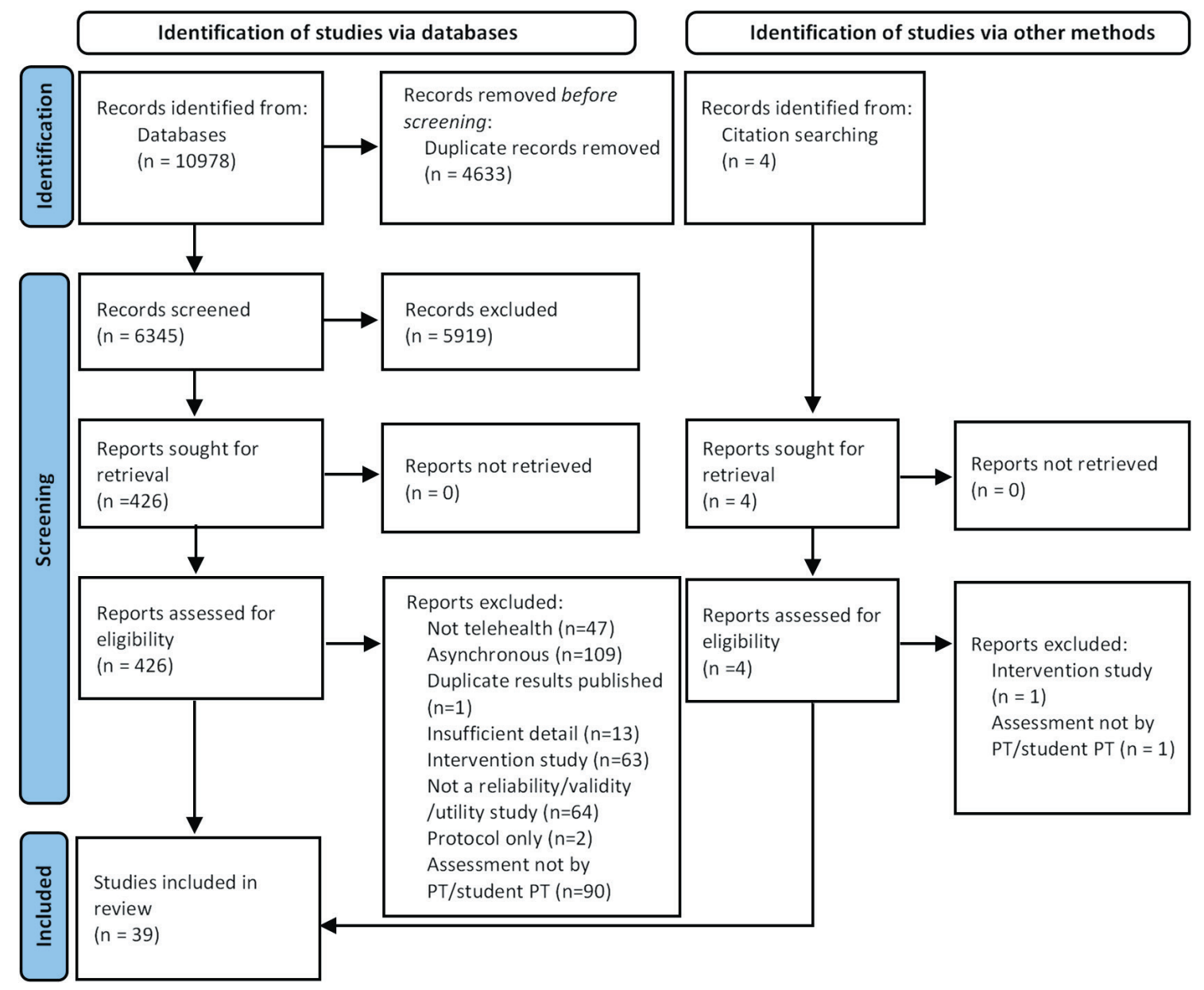

Figure 1. PRISMA flow diagram summarising results of the search, screening and selection processes [30].

Among the studies that investigated the concurrent validity of conducting a telehealth assessment when compared to a face-to-face assessment $(n=15)$, some failed to explain the face-to-face assessment $(n=3)$ or it's execution in enough detail to allow replication $(n=6)$. Most studies $(n=13)$ included a face-to-face assessment that was completed independent of the telehealth assessment and all studies considered a time frame between the two assessments that was short enough to limit changes in assessment findings.

Eleven of the included studies investigated the inter-rater and/or intra-rater reliability of performing a physiotherapy assessment via telehealth. Most study raters were blinded to the results of other raters $(n=10)$. Only one of the nine intra-rater reliability studies mentioned that raters were blinded to their own findings. The order of the examinations was varied in nine of the eleven studies. Three studies investigated inter-rater agreement between a clinician conducting a physiotherapy assessment via telehealth and a clinician conducting the same assessment face-to-face $[43,45,67]$. These studies demonstrated either good $(n=1)$ or very good $(n=2)$ methodological quality. One study did not discuss the reference standard in detail, and another failed to blind raters to each other's findings or set an appropriate time interval for test replication. Further details of the quality appraisals for the validity and reliability studies included in this review can be found in Table $S 2$ in the Online Supplementary Document).

Twenty-one of the reviewed studies were broader utility studies, which reported on the feasibility and outcomes of conducting telehealth assessments across a range of physiotherapy practice areas. Twelve of the utility studies were appraised using the MMAT [36] and nine were case studies or descriptive papers which were not appraised (Table 4). Similarly, one further utility study [64] included in this review was not appraised as it did not meet the screening criteria for the MMAT. The studies that were not appraised were considered to provide only low level (IV) evidence.

The methodological quality of the utility studies that were appraised varied from 'poor' $(\mathrm{n}=2)$ to 'very good' $(n=6)($ Table 4). Five studies used a qualitative design which appeared to be appropriate for the study question. Some qualitative studies provided limited information, making it difficult to determine whether the findings 


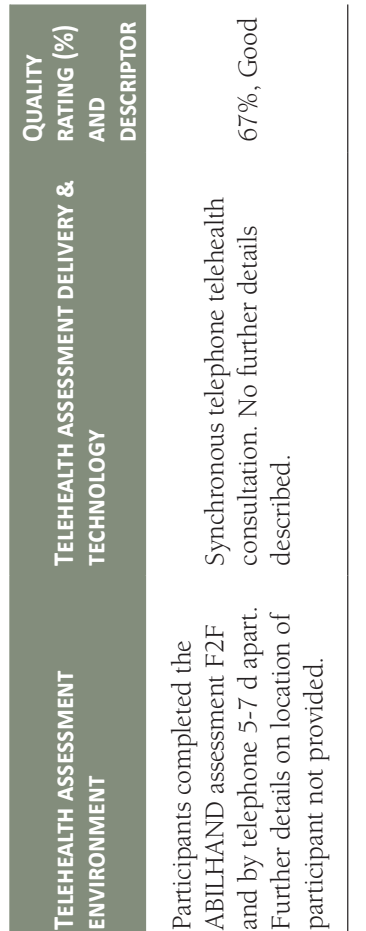

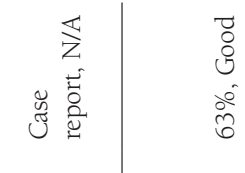

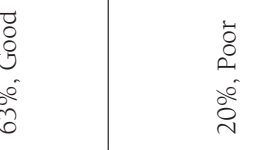

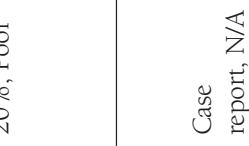

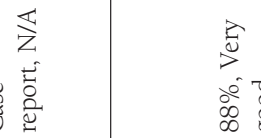

i

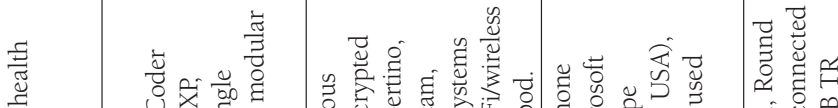

$\frac{\sqrt{5}}{\tilde{g}}$

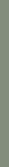

تี

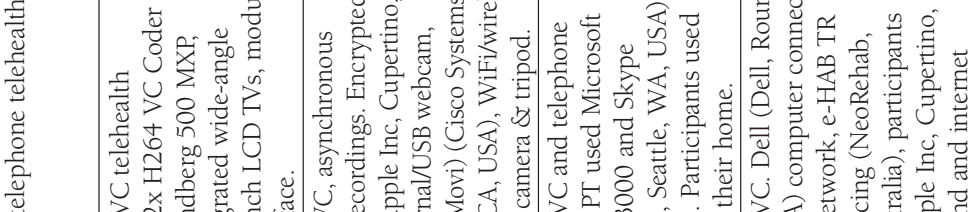

घ)

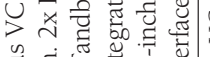

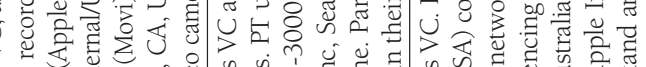

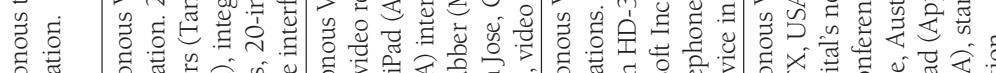

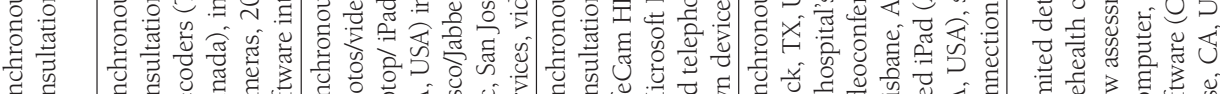

麇

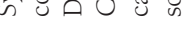

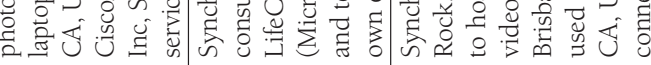

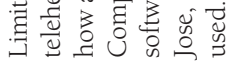

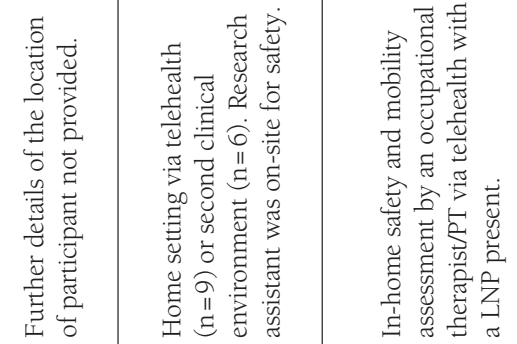

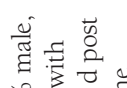
ถั)

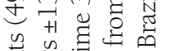

政

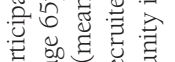

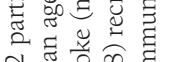

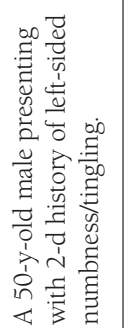

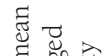

E

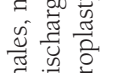

要

芯 苟

完不

可

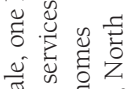

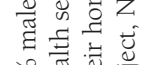

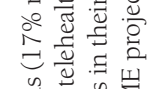

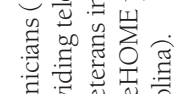

春它造曹

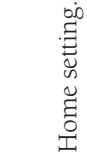

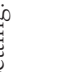

$\therefore \Xi$

焉

논

远岕

可

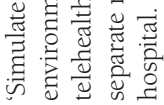

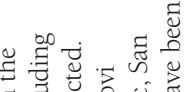

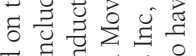

चु

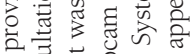

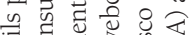

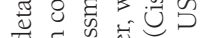

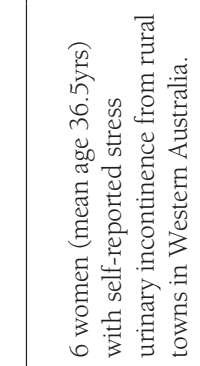

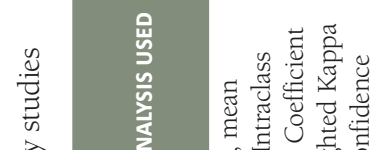

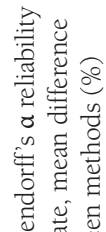

急

믈

产

는

竞

寻

y

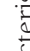

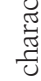

tin

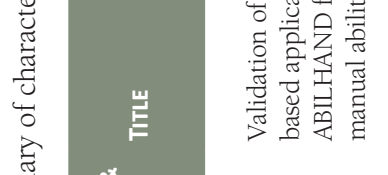

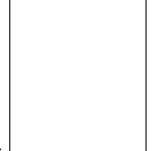

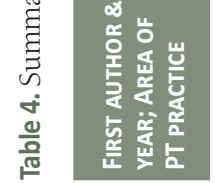

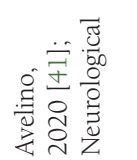

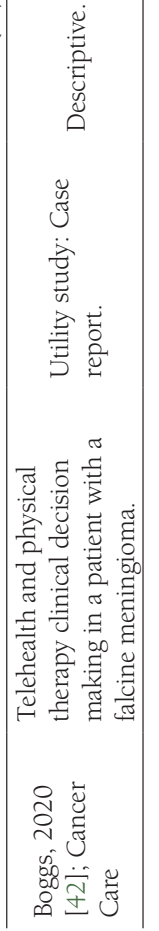

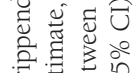

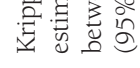

苞 苛

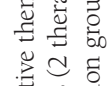

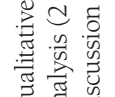

声

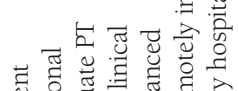

๘

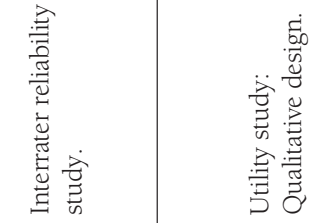

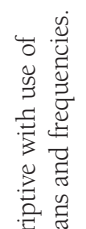

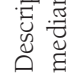

है है है

夌志

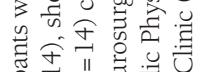

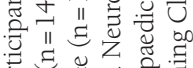

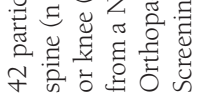

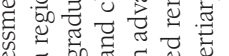

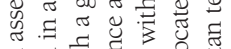

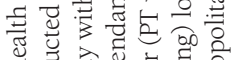

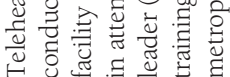

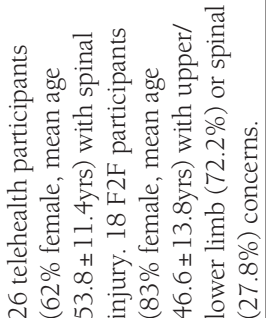

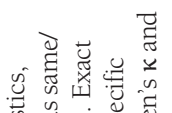

क्ष

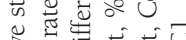

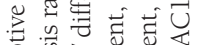

氙

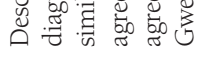

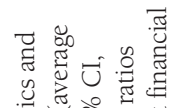

政品

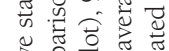

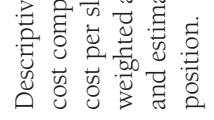

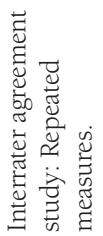

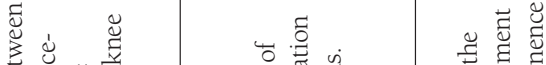

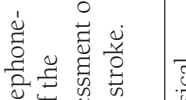

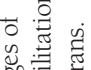

要要

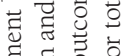

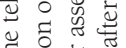

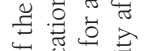

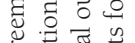

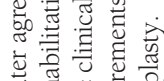

氜

可

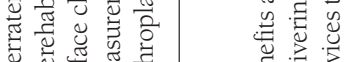

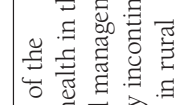

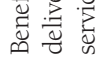

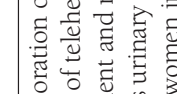

苛

$\approx$

표 8

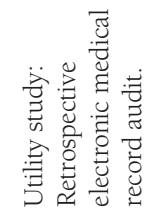

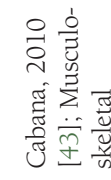

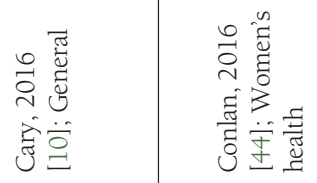

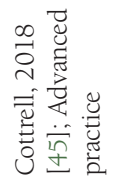

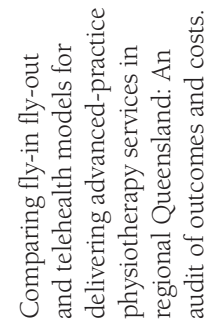




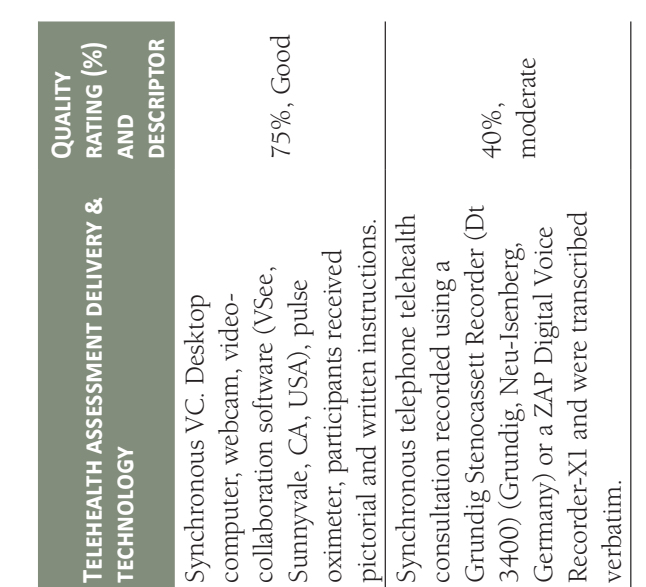

表密

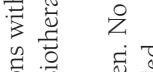

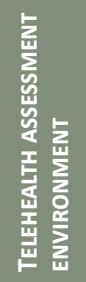

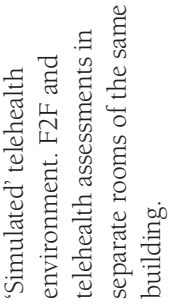

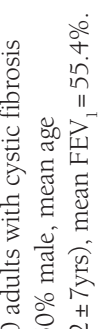

要贾

密 च

해을

苍危哭

vi

象

䨌

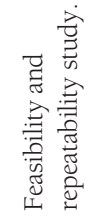

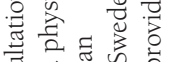

जू

8े

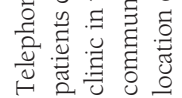

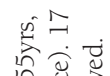

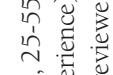

㐘

要

8 恶

点㲾泀

चี

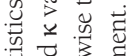

㯊 空

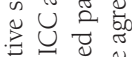

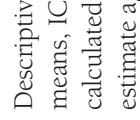

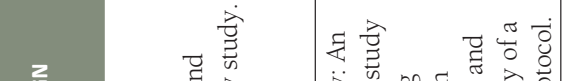

厸

高

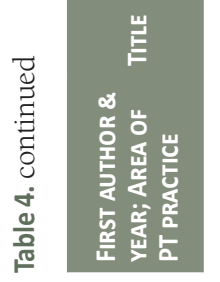

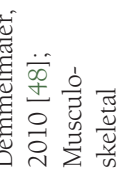

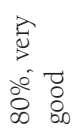

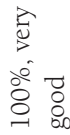

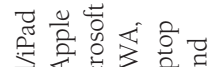

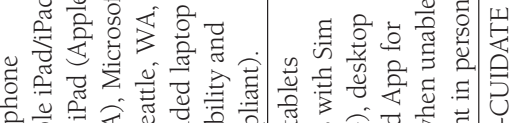

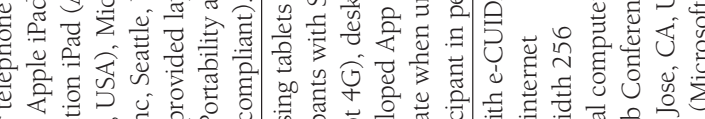

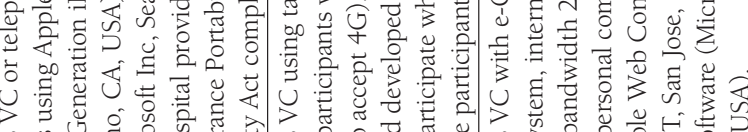

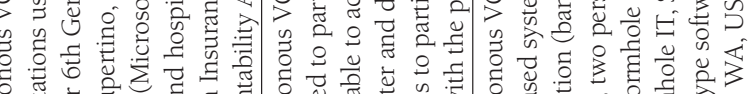

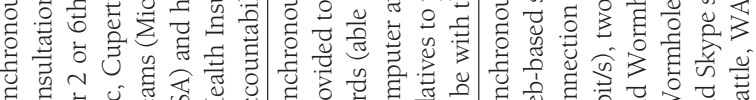

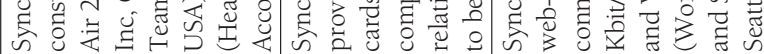

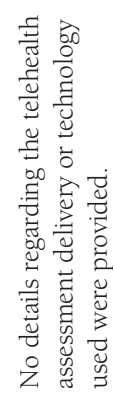

$\Xi \frac{9}{1}$

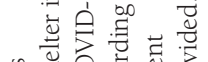

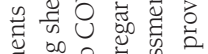

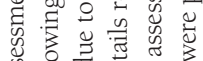

为

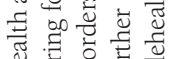

กิ

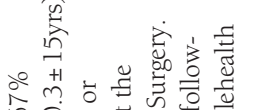

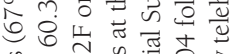

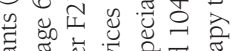

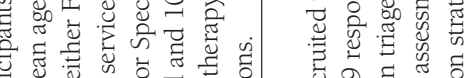

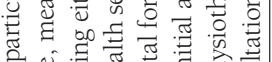

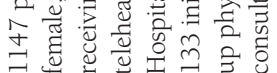

$\vec{\sigma}$ 品

तै

उ

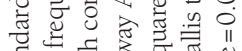

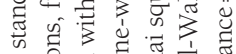

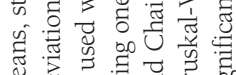

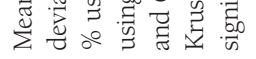

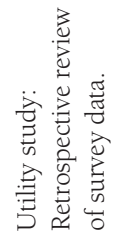

衰嵒

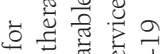

ธี चٓ

造

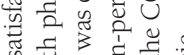

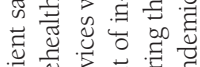

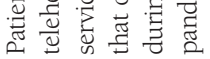

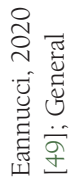

ซ

品

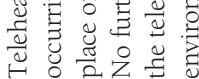

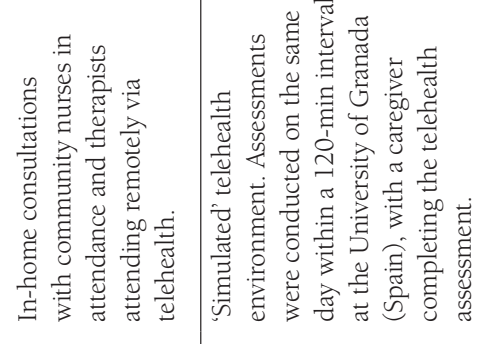

हี

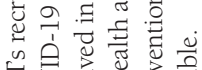

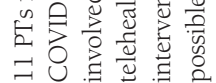

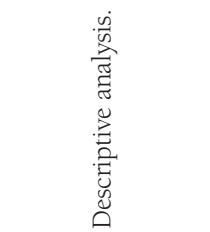

总焉

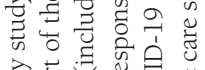

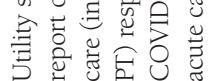

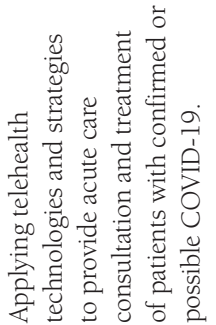

2 $\quad$ ह है

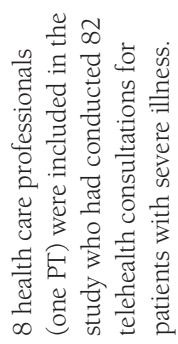

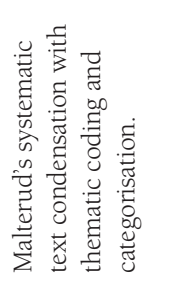

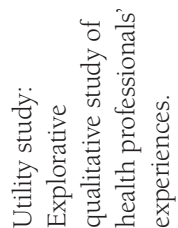

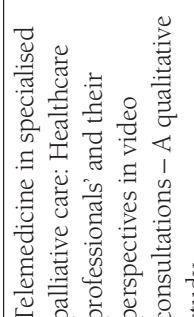

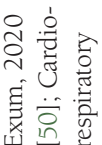

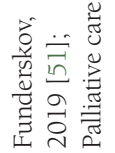

急 焉

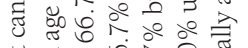

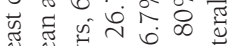

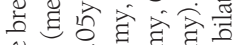

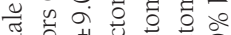

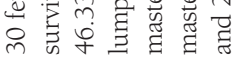

๑ $\quad$ :

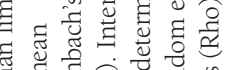

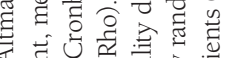

约

च

ल

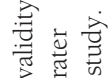

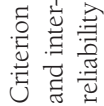

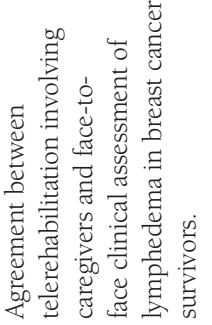

怘

웜

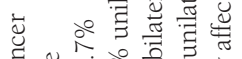




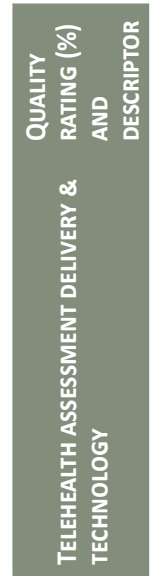

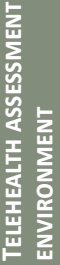
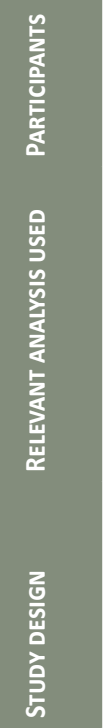

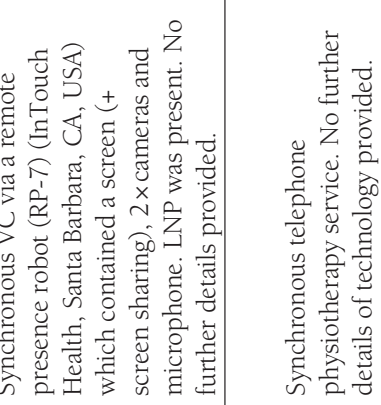

)

to

官苛它

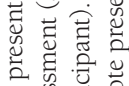

兄岕.

我究

๘

3 :

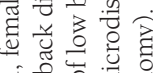

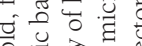

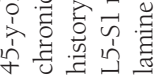

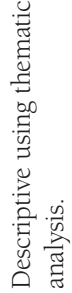

$\ddot{g}$

홀

氞莒

\%ँ

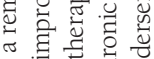

of

涉

붕응를

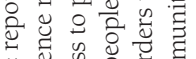

亗

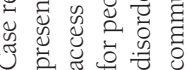

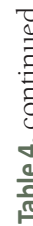

क

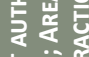

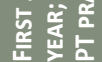

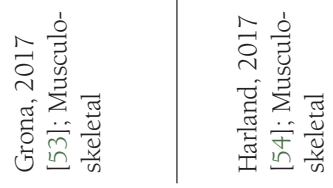

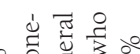

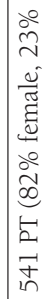

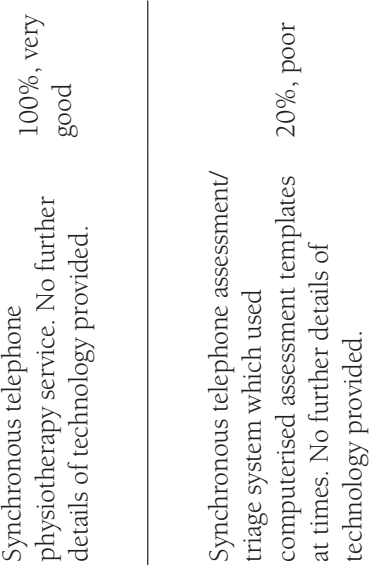

$\widetilde{\nabla} \overrightarrow{0}$

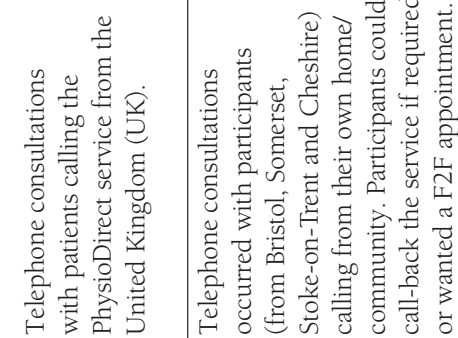

离䓂旁

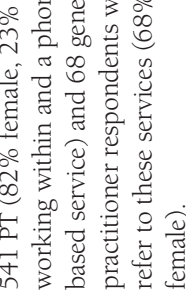

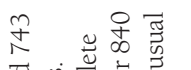

च्

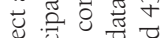

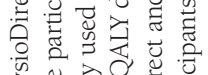

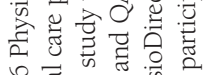

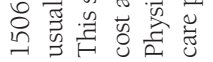

\section{y.}

प

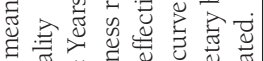

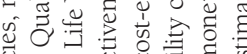

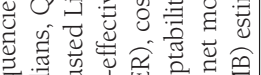

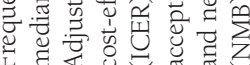

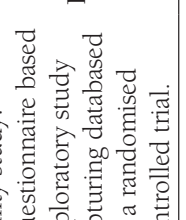

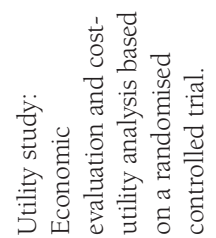

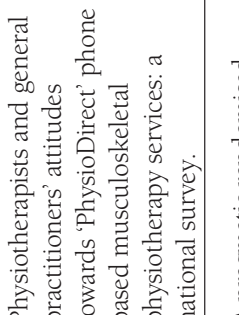

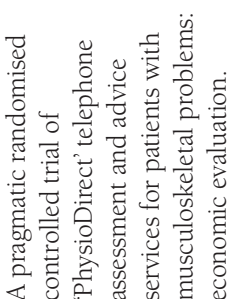

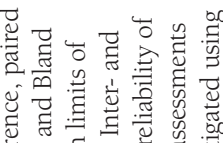

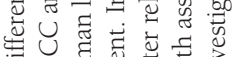

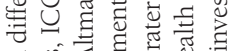

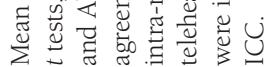

苛

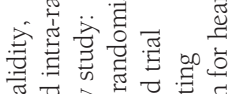

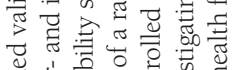

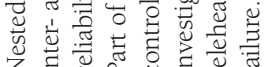

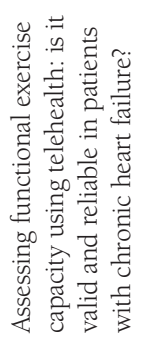

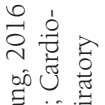

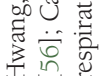

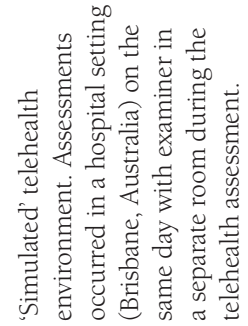

艺芯

7
8
8
$\circ$
0
0

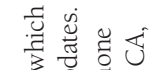

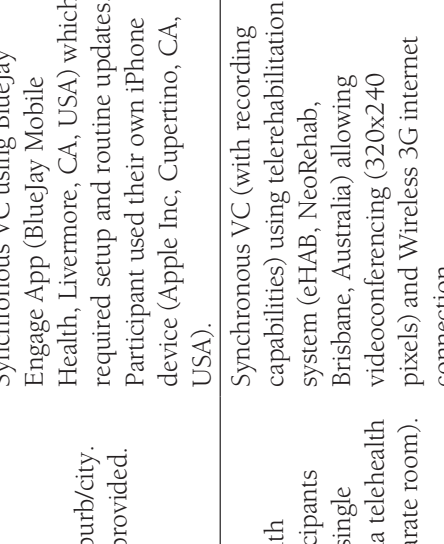

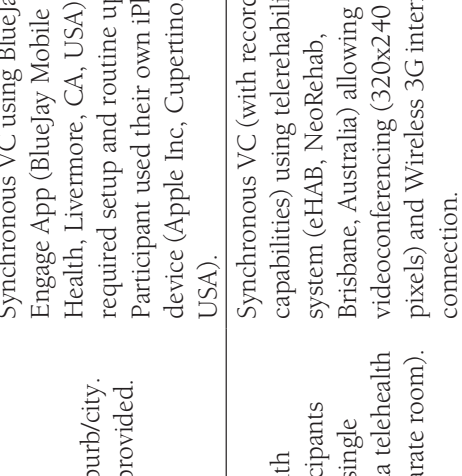

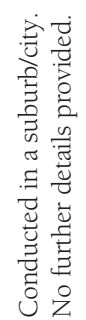

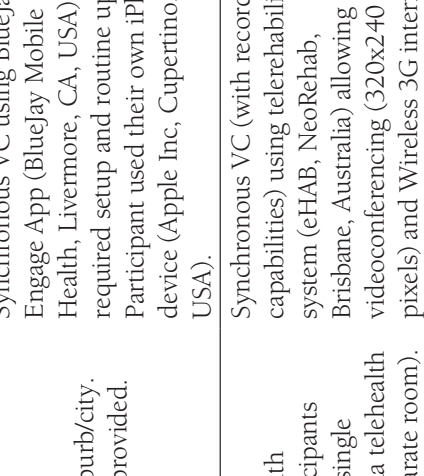

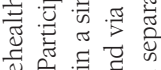

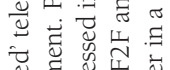

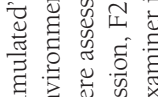

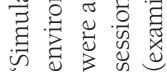

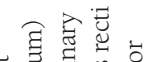

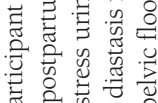

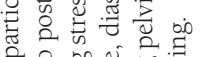

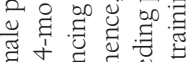

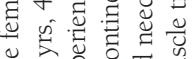

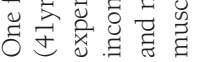

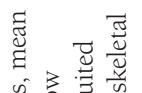

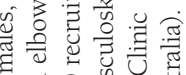

决

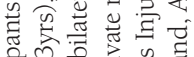

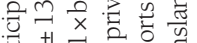

范市

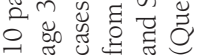

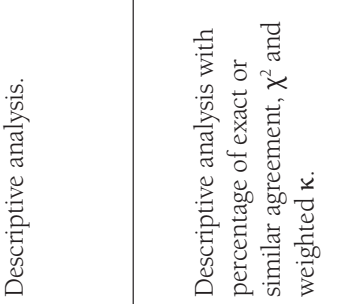

\section{(3)}

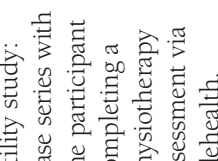

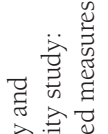
记密

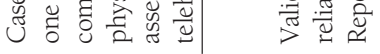

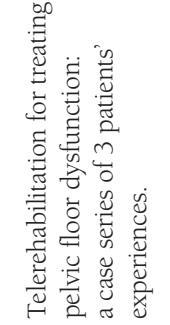

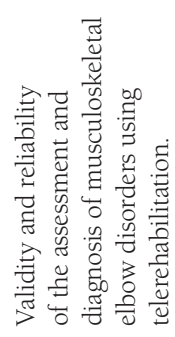

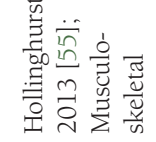

踖

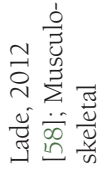




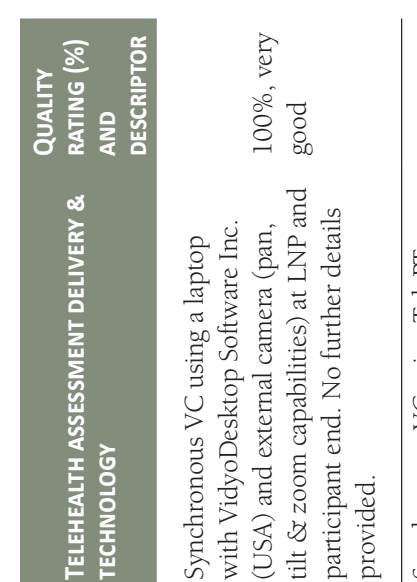

II

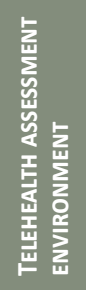

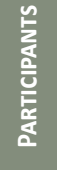

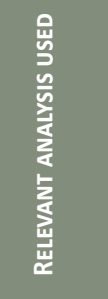

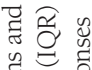

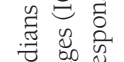

藏

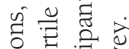

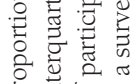

这彭䇏哭

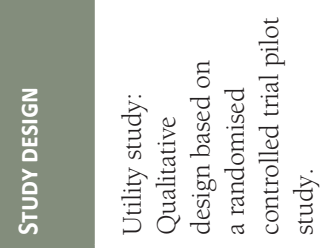

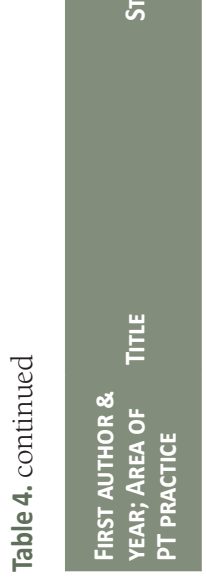

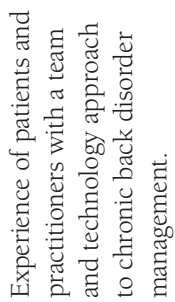

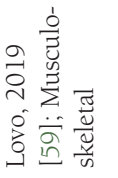

装

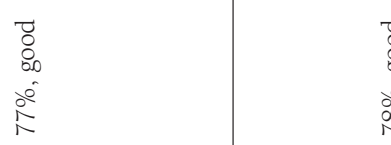

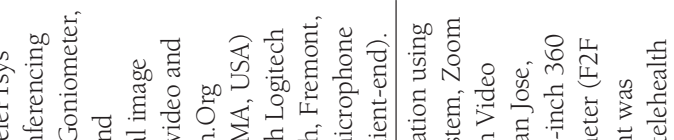

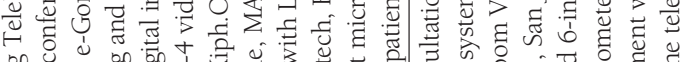

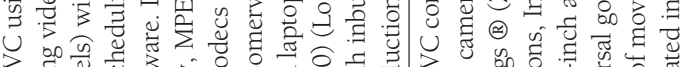

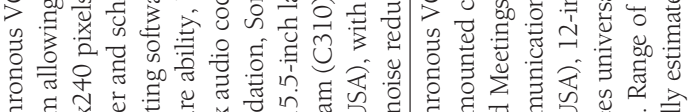

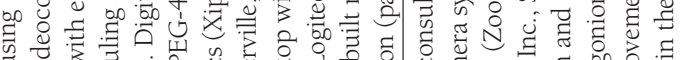

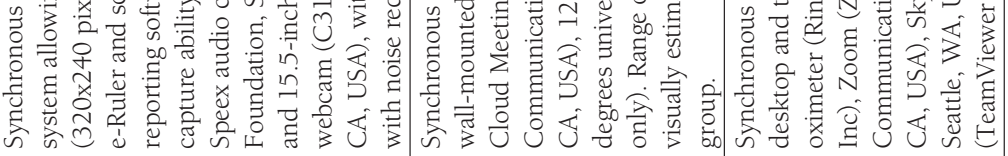

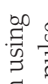

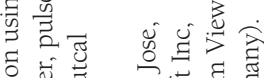

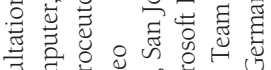

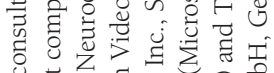

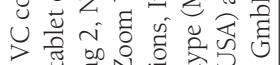

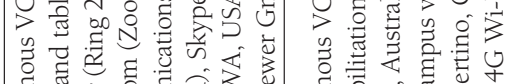

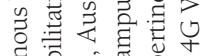

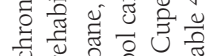

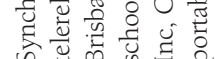

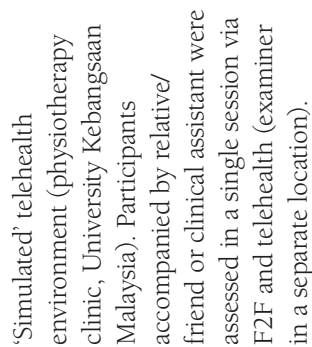

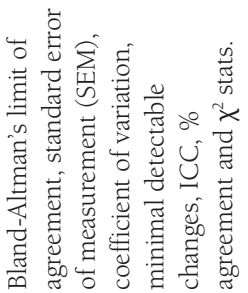
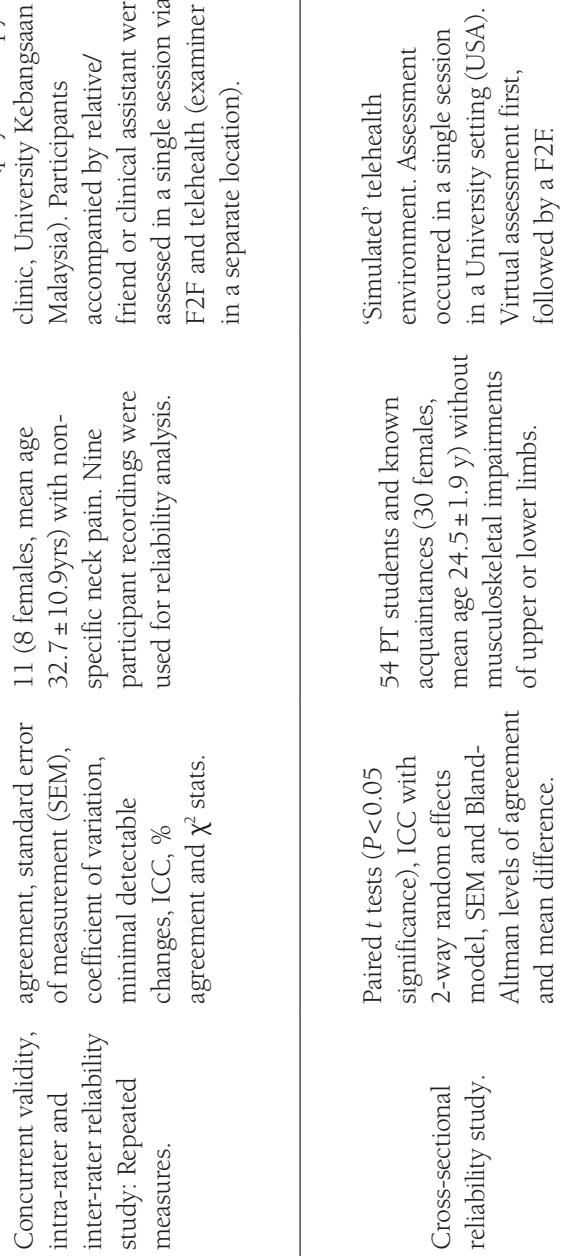

总

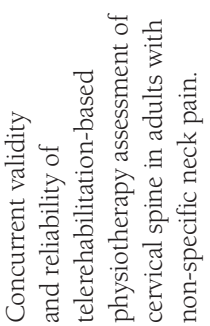

突

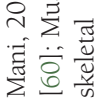

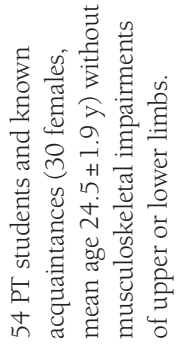

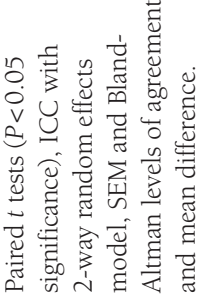

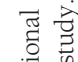

空

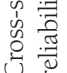

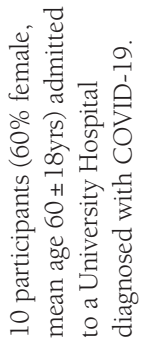

章

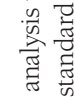

着蒙要

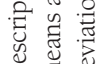

0 घ

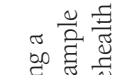

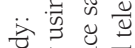

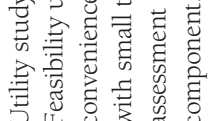

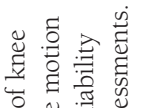

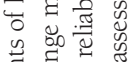

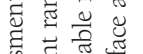

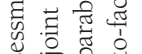

要言

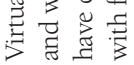

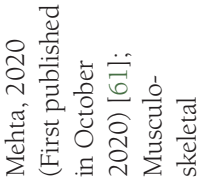

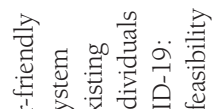

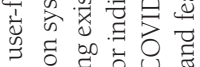

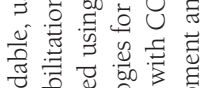

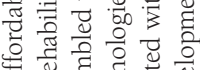

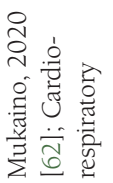

跑

흥

흘

䨌

馬

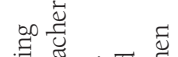

苞

홍응

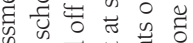

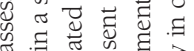

들

可

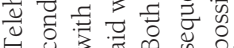

बक्षे

bo

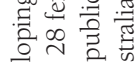

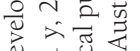

쿵

चี

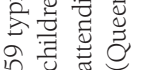

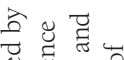

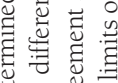

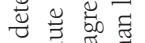

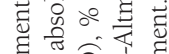

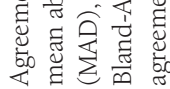

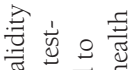

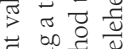

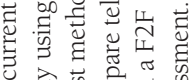

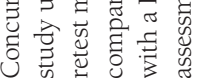

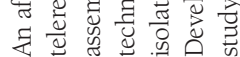

\section{莀}

然

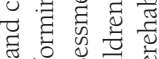

실

要

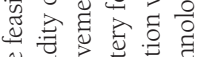

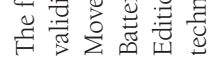

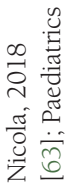




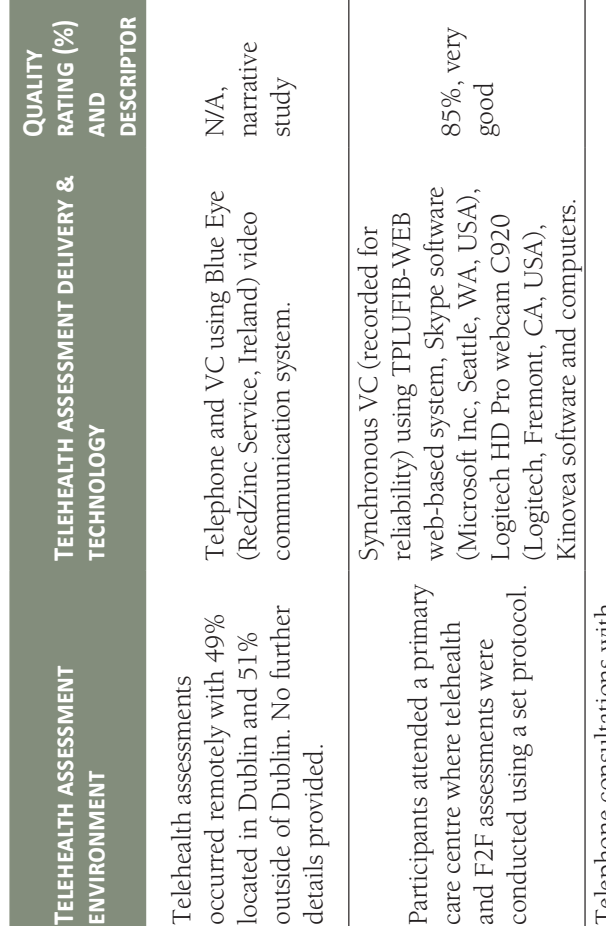

تี

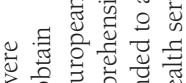

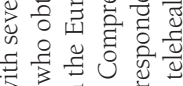

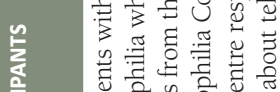

泀运言苟

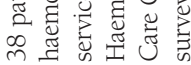

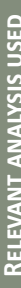

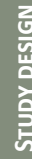

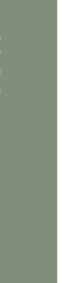

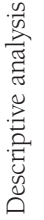

능

$\overline{5}_{0}$

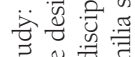

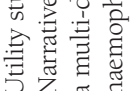

5 Zू

ธั

형

의

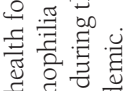

崖

,

$\infty$

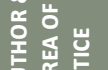

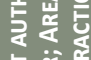

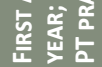

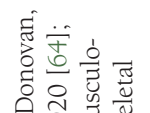

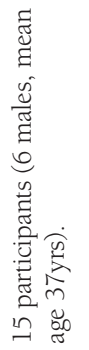

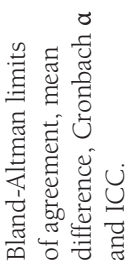

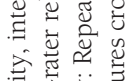

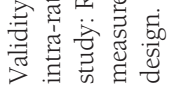

芩苋

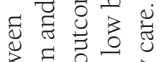

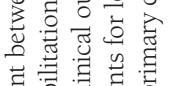

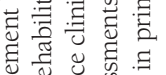

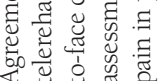

离

동

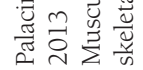

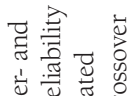

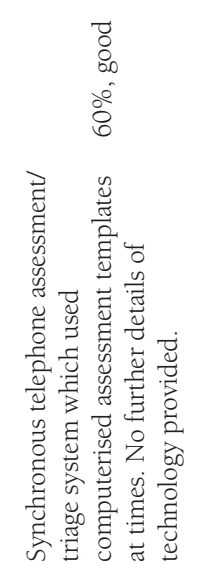

है चี

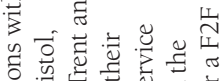

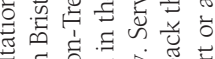

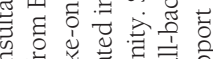

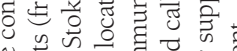

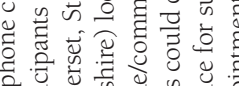

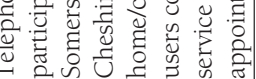

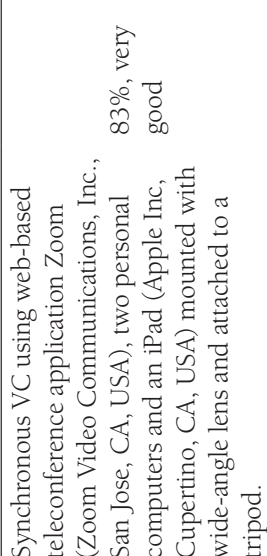

总

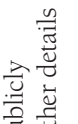

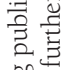

莺之

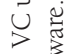

号起

氙造

空

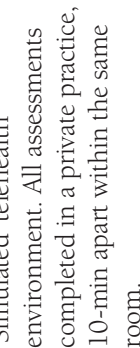

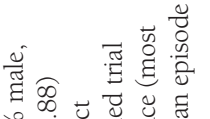

员足.

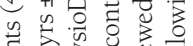

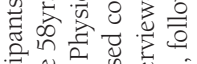

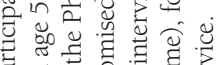

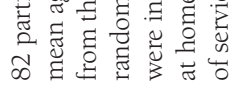

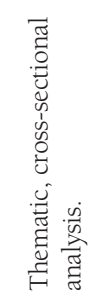

苟

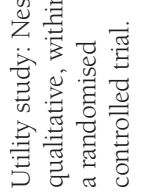

范

密泀

$\circ$ 纯

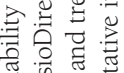

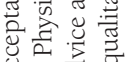

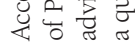

일

总焉

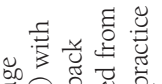

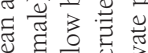

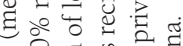

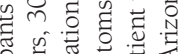

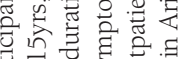

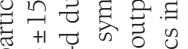

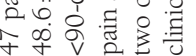

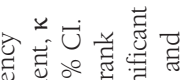

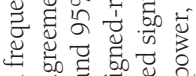

战

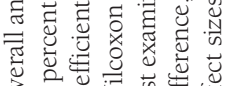

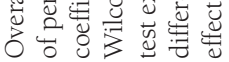

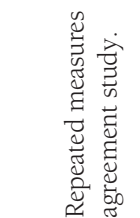

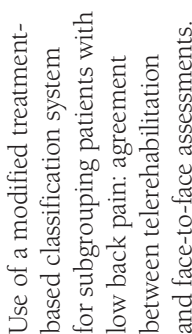

空

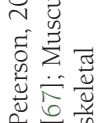

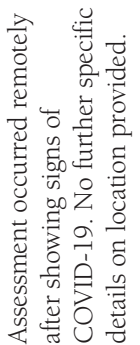

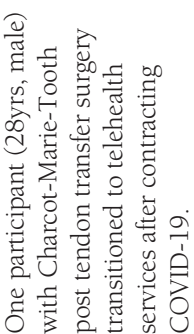

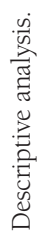

岁

氡

言莒

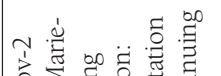

论

击

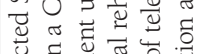

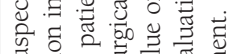

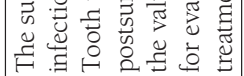

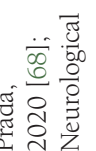

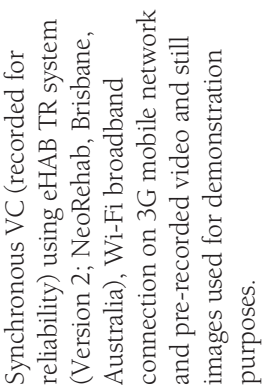

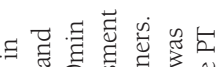

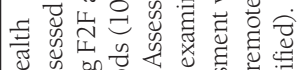

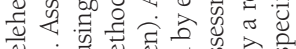

苛

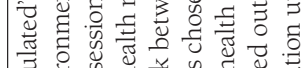

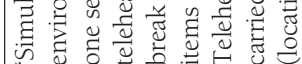

胥: 芯

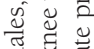

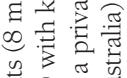

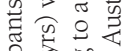

臭令品

플 $\approx \tilde{y}$

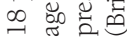

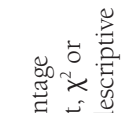

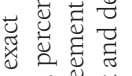

证

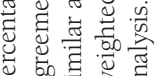

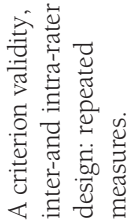

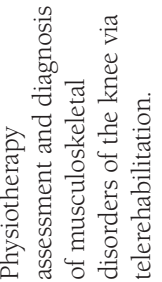

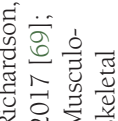




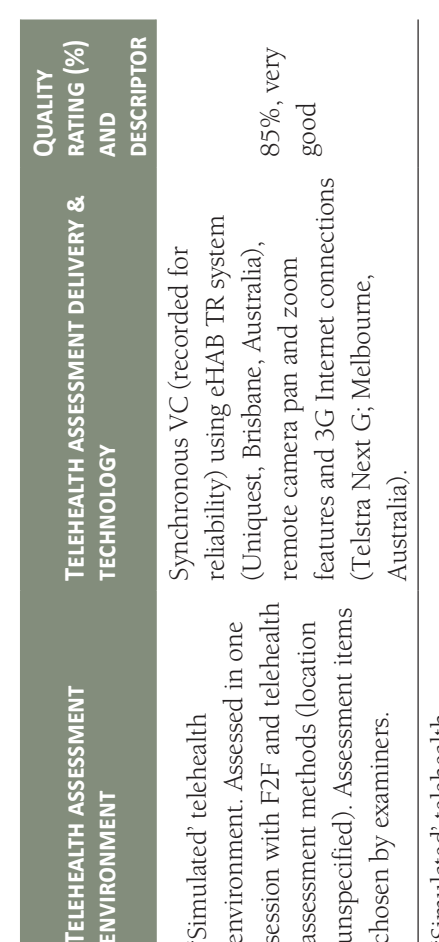

䓃至
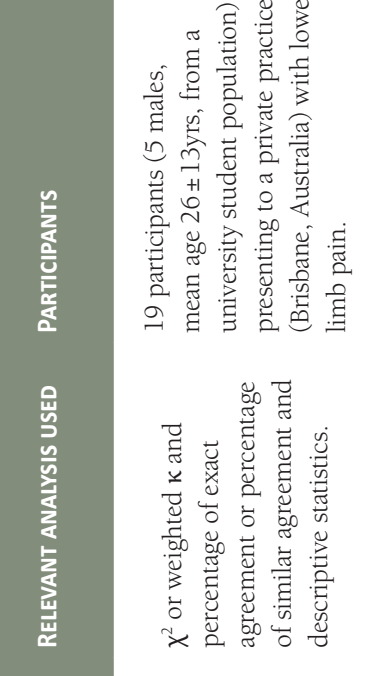

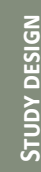
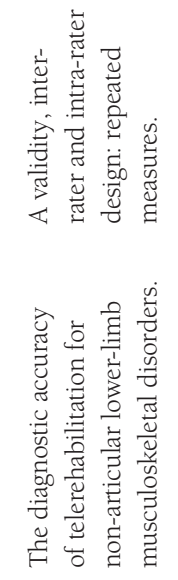

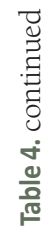

崖

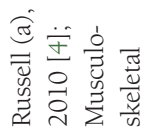

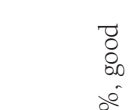

$\stackrel{8}{8}$

घ

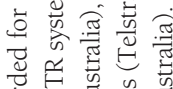

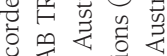

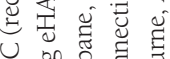

$>$ bo

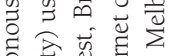

奢

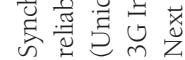

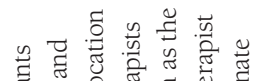

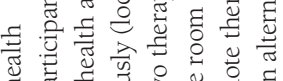

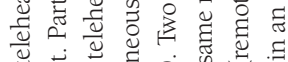

芩

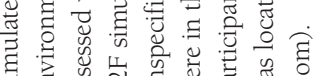

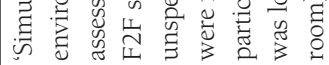

옥ㄹ

药藏总

oํ

他

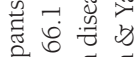

氖

苋氜

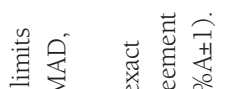

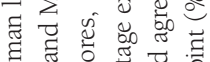

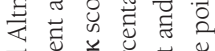

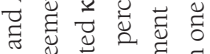

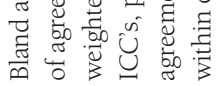

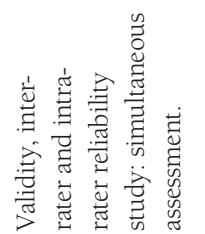

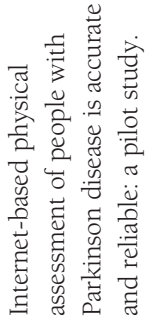

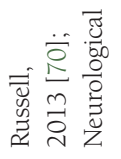

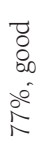

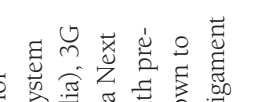

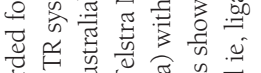

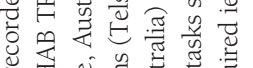

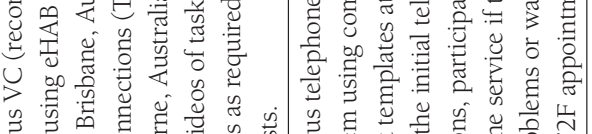

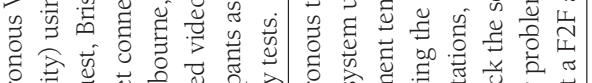

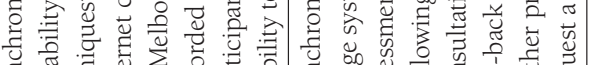

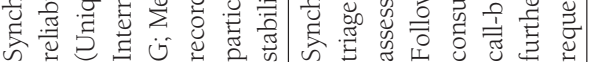

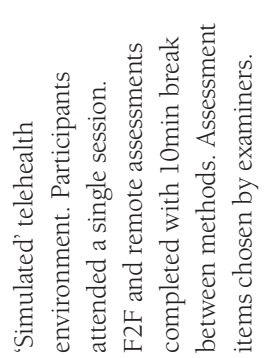

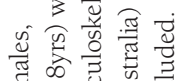

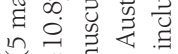

范药
运焉苛

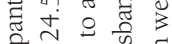

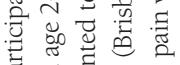

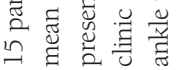

苂

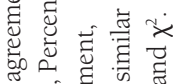

叫

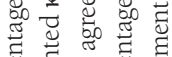

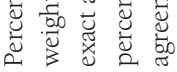

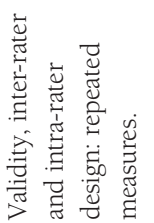

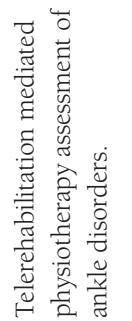

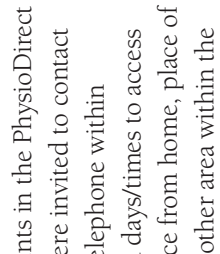

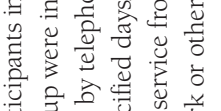

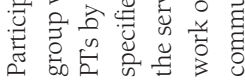

卺

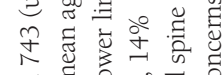

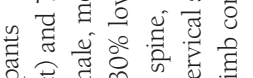

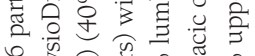

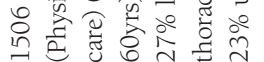

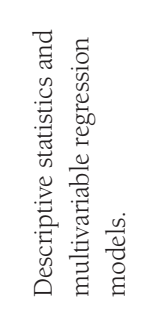

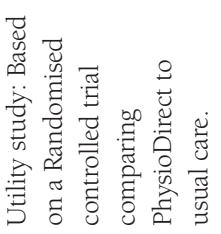

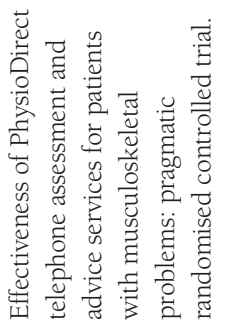

角

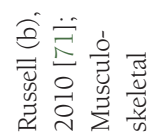

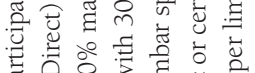

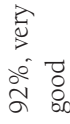

莺结

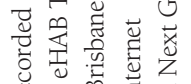

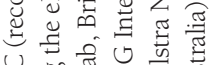

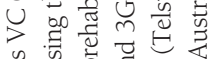

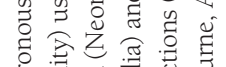

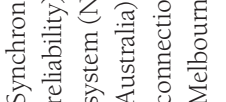

ี ฮ 


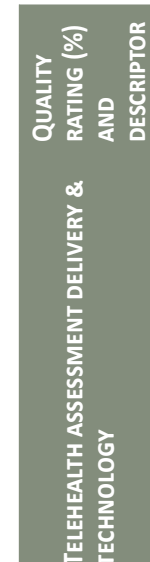

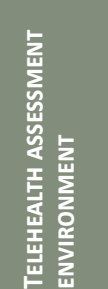

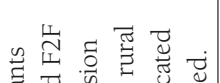

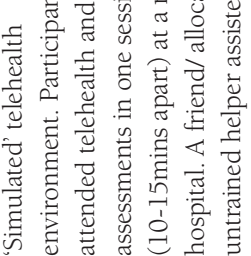

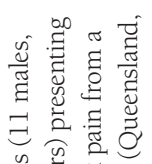

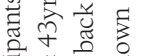

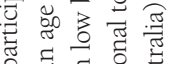

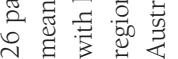

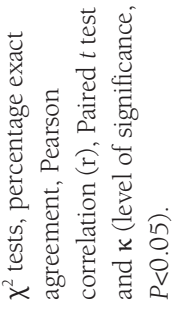

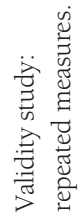

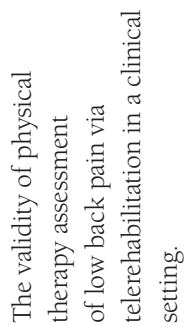

萿

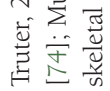

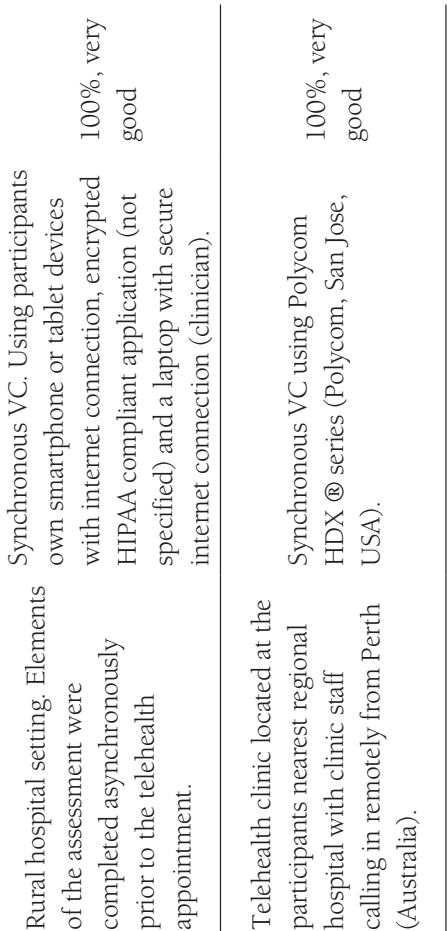

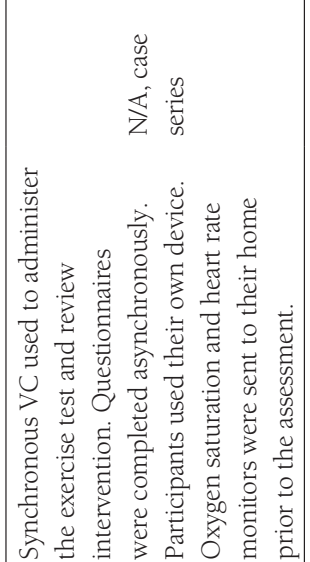

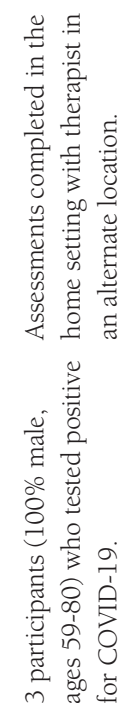

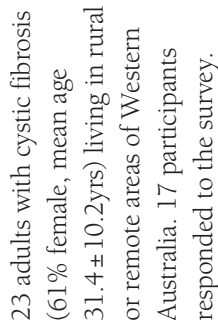

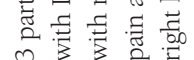

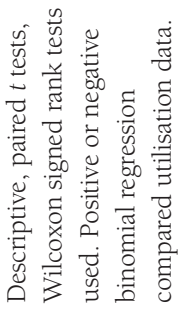

$\stackrel{0}{00}$

匍

言客

昰愛

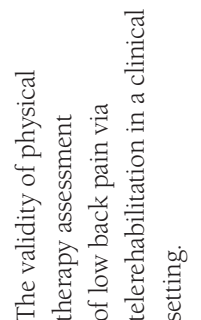

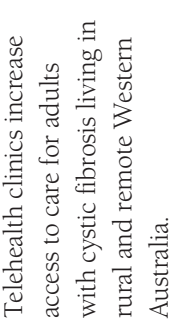

象

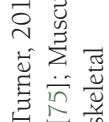

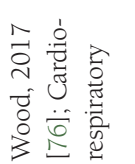

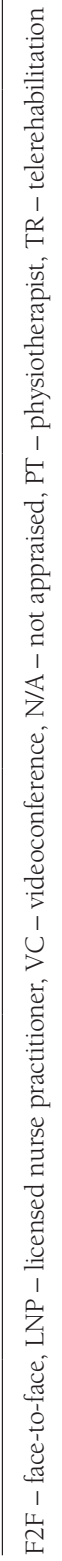


were adequately derived $(n=2)$ or if results were authenticated from the data $(n=3)$. Further details of the results of the MMAT quality appraisals for the utility studies included in this review can be found in Table S3 in the Online Supplementary Document).

\section{Study characteristics}

A summary of the characteristics and findings of the included studies is provided in Table 4 and Table 5. These studies investigated the validity, reliability or broader utility of synchronous physiotherapy assessments performed via telehealth across a variety of clinical practice settings and populations, which included children and adult participants with and without motor impairments. Sixty-four percent of the studies were published over the past five years. The number of participants in each included study varied, with nine validity/reliability studies including less than 20 participants. Two larger studies investigated synchronous, telehealth physiotherapy assessments conducted in healthy populations $[61,63]$. Variability was also observed across the utility studies, with participant numbers ranging from one (case studies) to 2249 [72]. There was inconsistency in the execution of telehealth assessments across practice settings, with some participants accompanied by a friend or relative $[50,60,74]$, teacher's aide [63] or health practitioner (nurse or less experienced physiotherapist) $[10,46,50,53,76]$ to assist with the assessment. The experience levels of clinicians conducting telehealth assessments also varied. Physiotherapy students under supervision performed telehealth assessments in some studies ( $n=6$ ), whilst others involved physiotherapists with extensive experience (up to 31 years) or post graduate training in specialised areas of practice $[45,46]$. Some clinicians had previous experience in performing virtual assessments or were provided with training prior to conducting the telehealth assessment, while others had none.

\section{Areas of practice}

The validity, reliability and utility studies included in this review were from a variety of physiotherapy practice areas (Figure 2). Four of these studies reported on the same 'PhysioDirect' (PD) telephone screening service and included participants presenting with a range of musculoskeletal conditions $[54,55,66,72]$.

\section{Telehealth assessment environment}

The type of environment in which the telehealth assessment occurred was either 'simulated' ( $n=15)$, which consisted of a university or clinic room set up for telehealth with the physiotherapist located in another room of the same building as the client, or 'real-world' $(n=24)$, where the client was located in a setting such as their home, school or second clinical environment and the physiotherapist was located elsewhere (Figure 3).

\section{Utility of telehealth assessments}

\section{Overall utility}

Aspects of telehealth utility explored across the studies in this review (Table 5) included telehealth assessments in rural and remote environments, satisfaction and attitudes of clients and health care professionals towards telehealth, clinical outcomes, consultation and assessment types completed, telehealth strategies employed, service access, and technology and economic evaluation. The studies which considered aspects of telehealth utility were set across a broad range of physiotherapy settings, including home, community, and hospital settings.

Nine of the utility studies were case studies or descriptive papers, with four of these reporting on telehealth use during the COVID-19 pandemic $[50,64,68,77]$. Despite some initial challenges with scheduling and rapid adoption of a new method of service provision, telehealth physiotherapy services for participants diagnosed with COVID-19 facilitated and maintained service access at times when isolation was required. One study reported $73 \%$ of physiotherapy consultations moved to telehealth (46\% telephone, $27 \%$ video conference) during the COVID-19 pandemic [64]. Strategies used to provide the telehealth assessments to patients with COVID-19 included delivery of pulse oximeters to the patient's home [77], development of a triage system, utilisation of nursing staff already in contact with the patient in isolation to assist with telehealth assessments [50], and use of functional tasks in lieu of formal assessment items to infer hand strength and dexterity following hand surgery [68].

One observational study [48] and the remaining case studies/series included in this review, investigated the use of telehealth assessments for participants with either low back pain $[48,53,75]$, gender health concerns (pelvic dysfunction or post-partum support) [44,57] or cancer care [42]. Of these studies, one study reported telehealth consultation lengths ranging from 3-21 minutes (mean 8.5 minutes) with consultation time devoted 

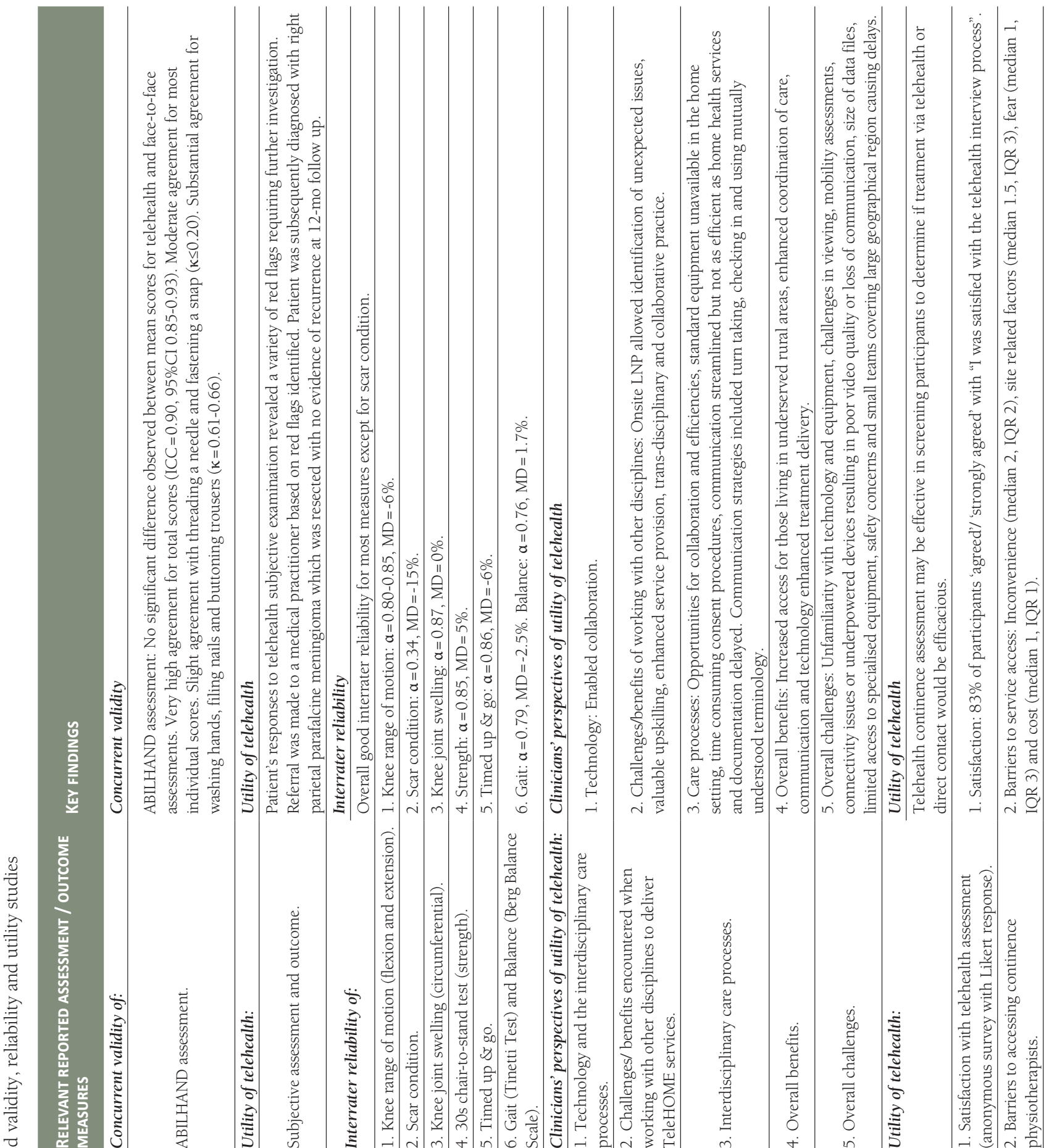

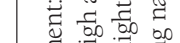
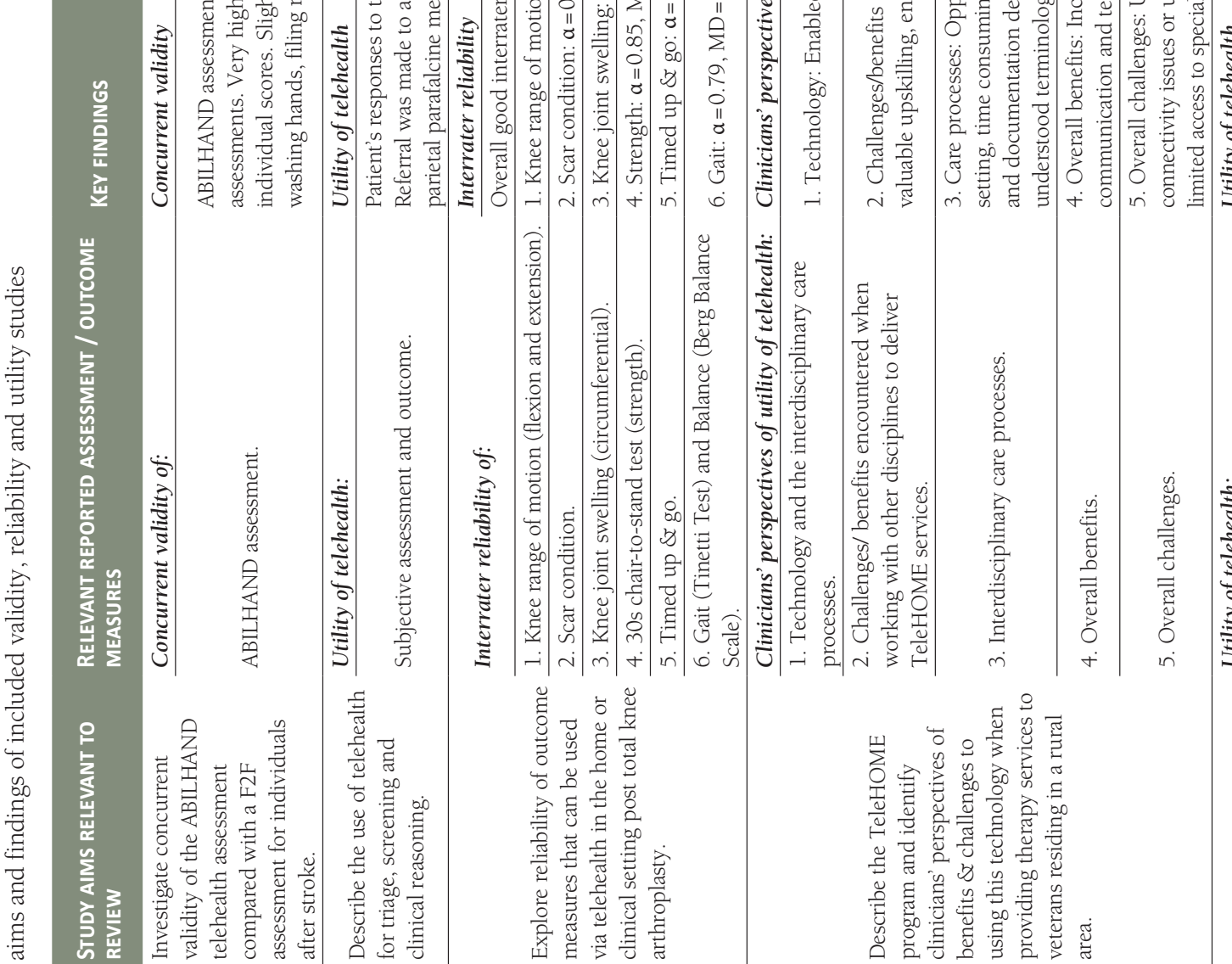

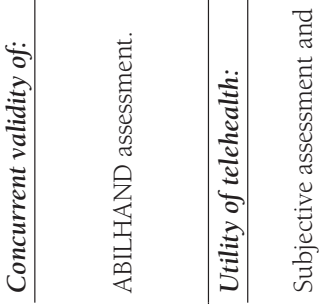

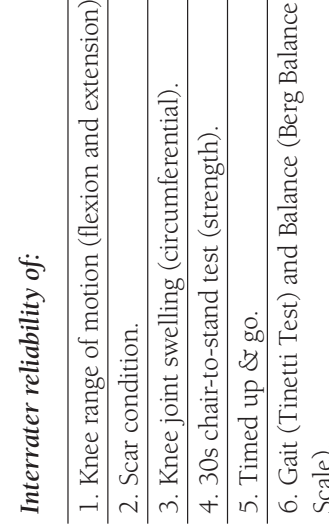

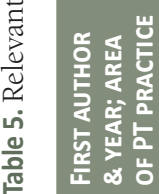

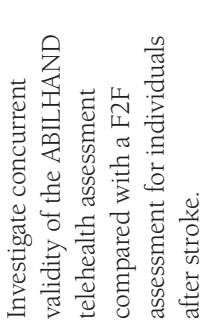

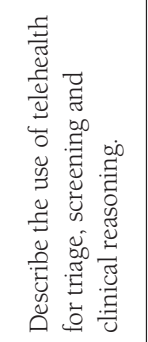

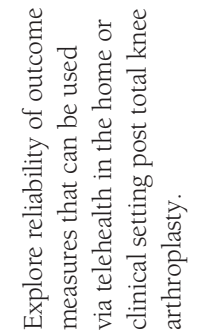

울 $\frac{1}{3}$

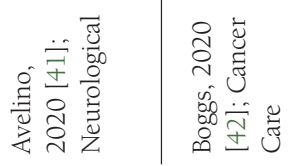

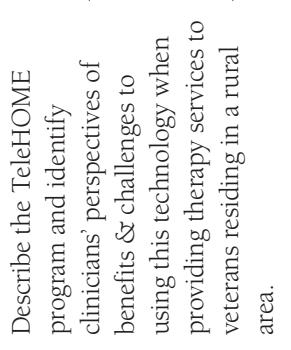

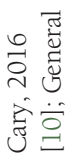
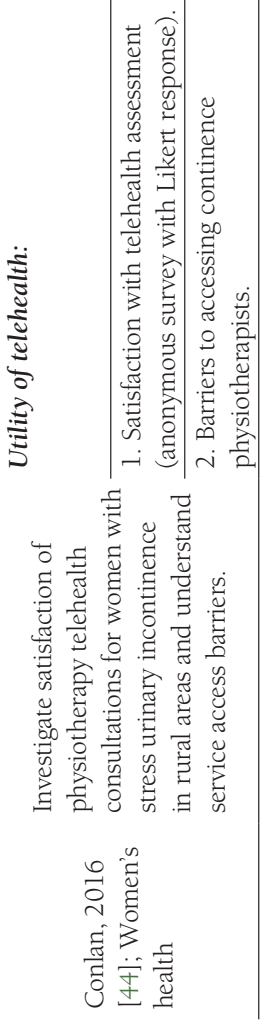


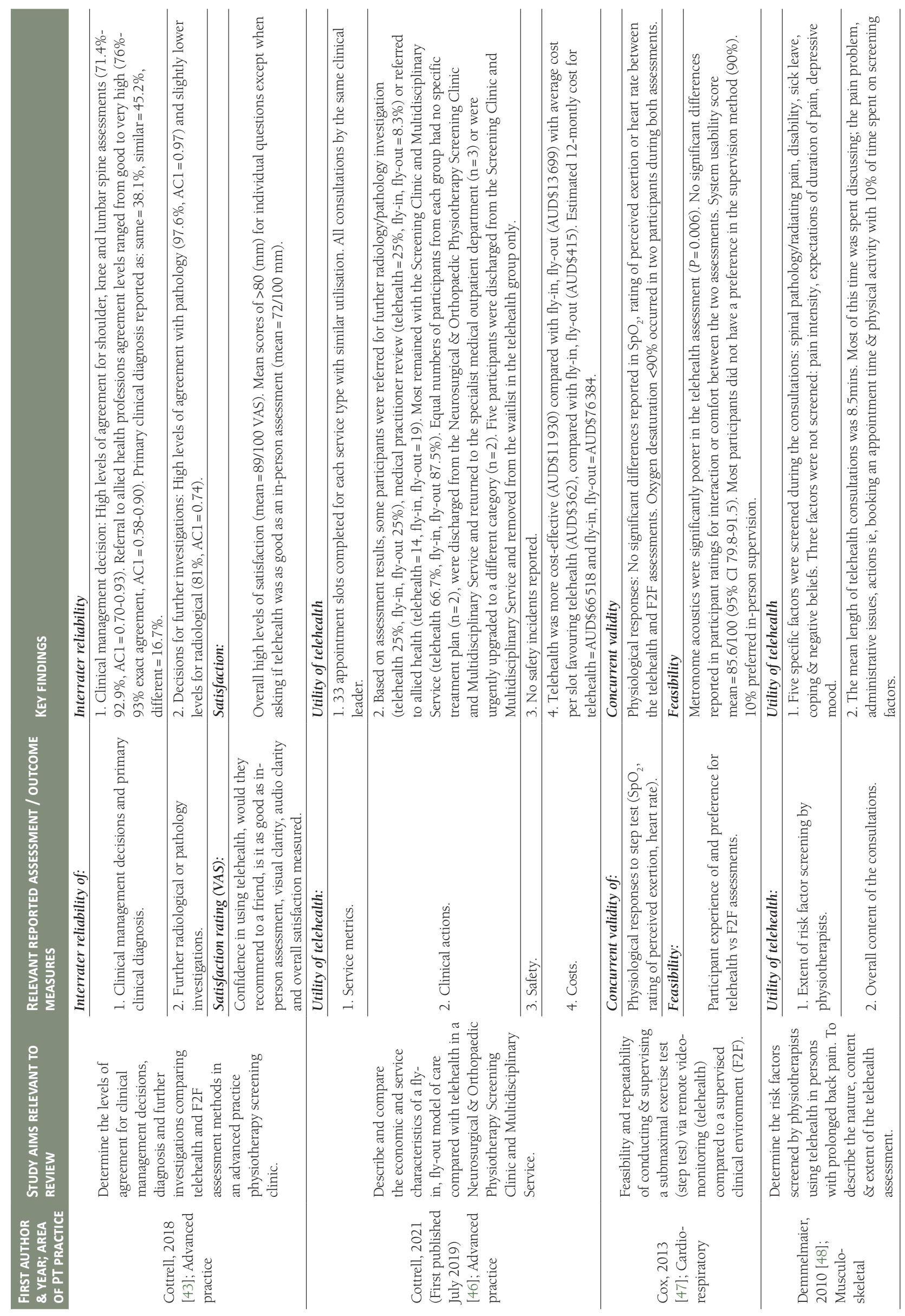



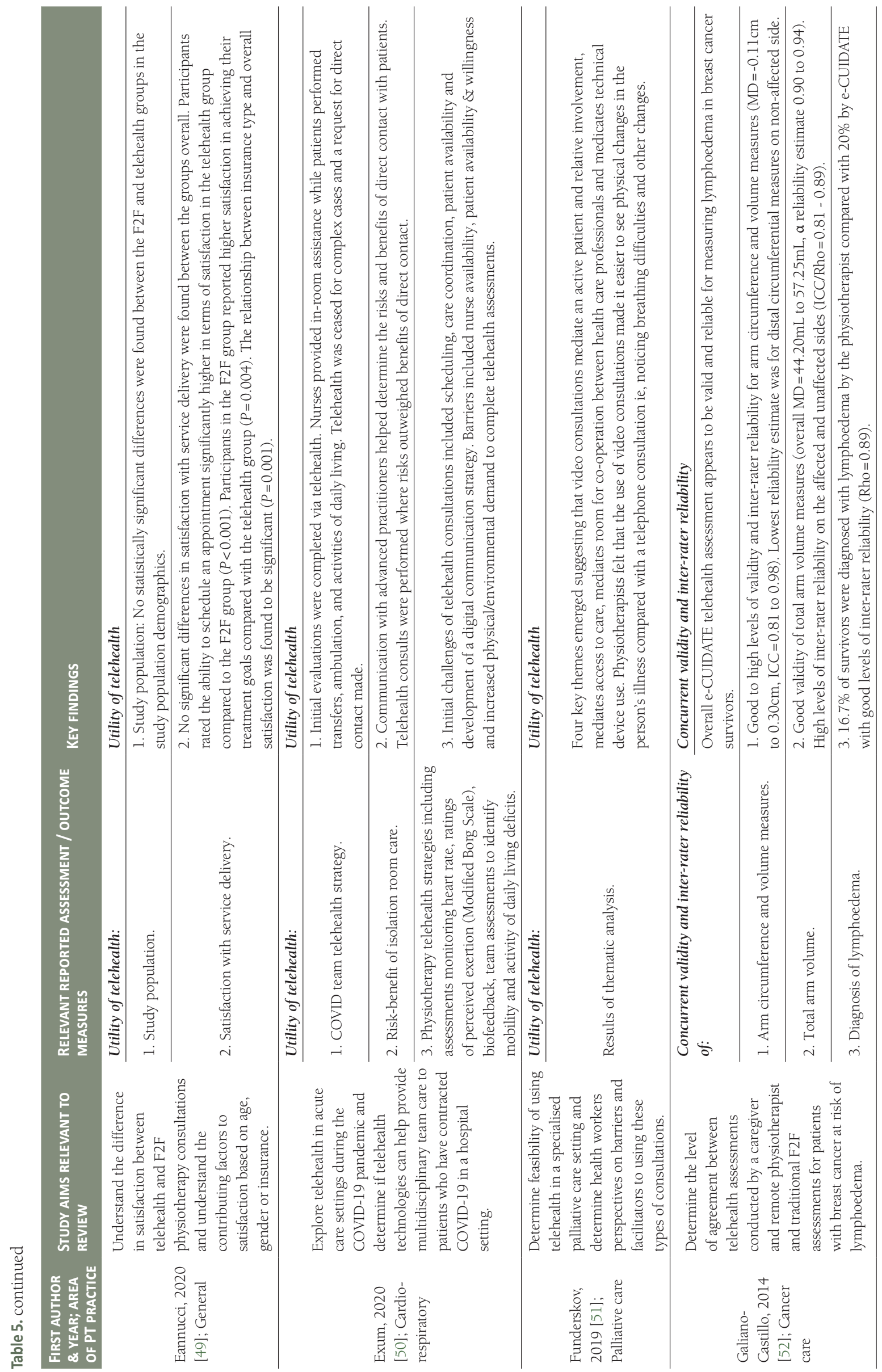


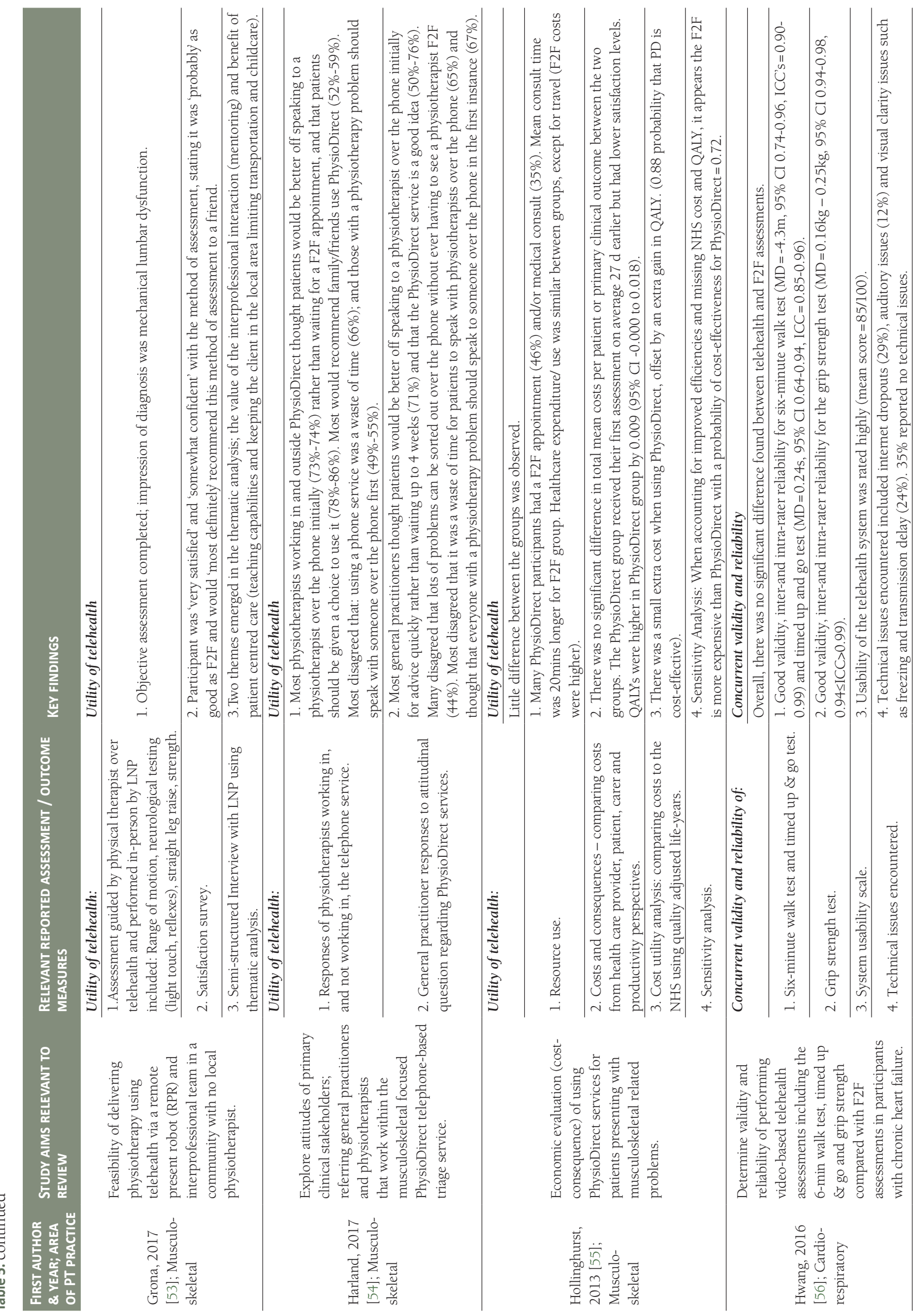




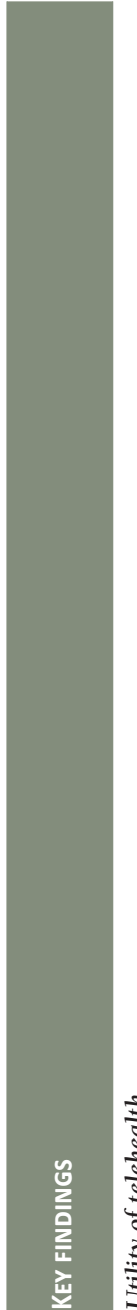

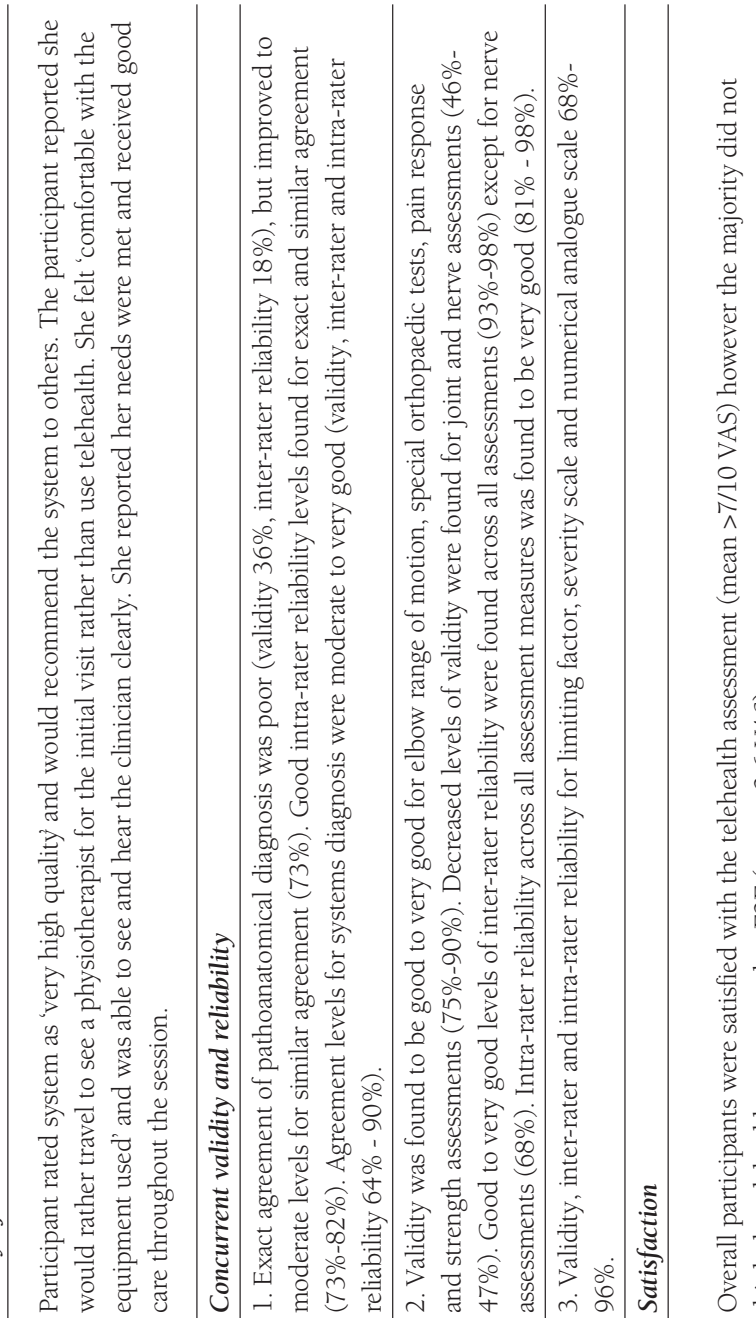
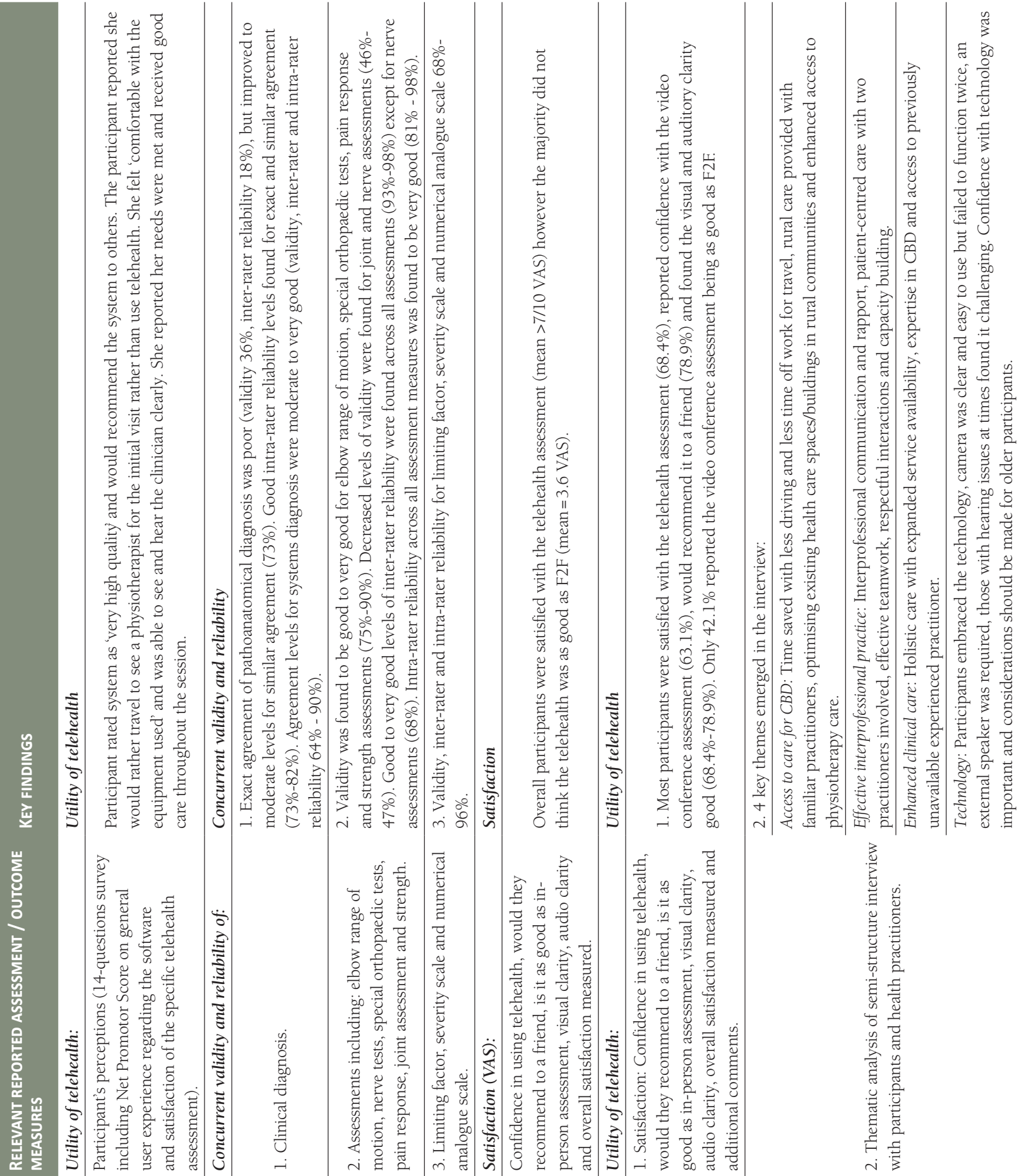

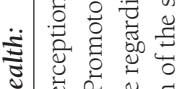

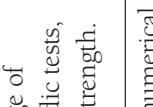

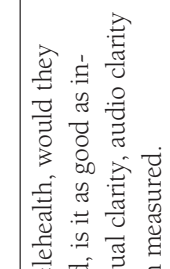

돈 i 突要
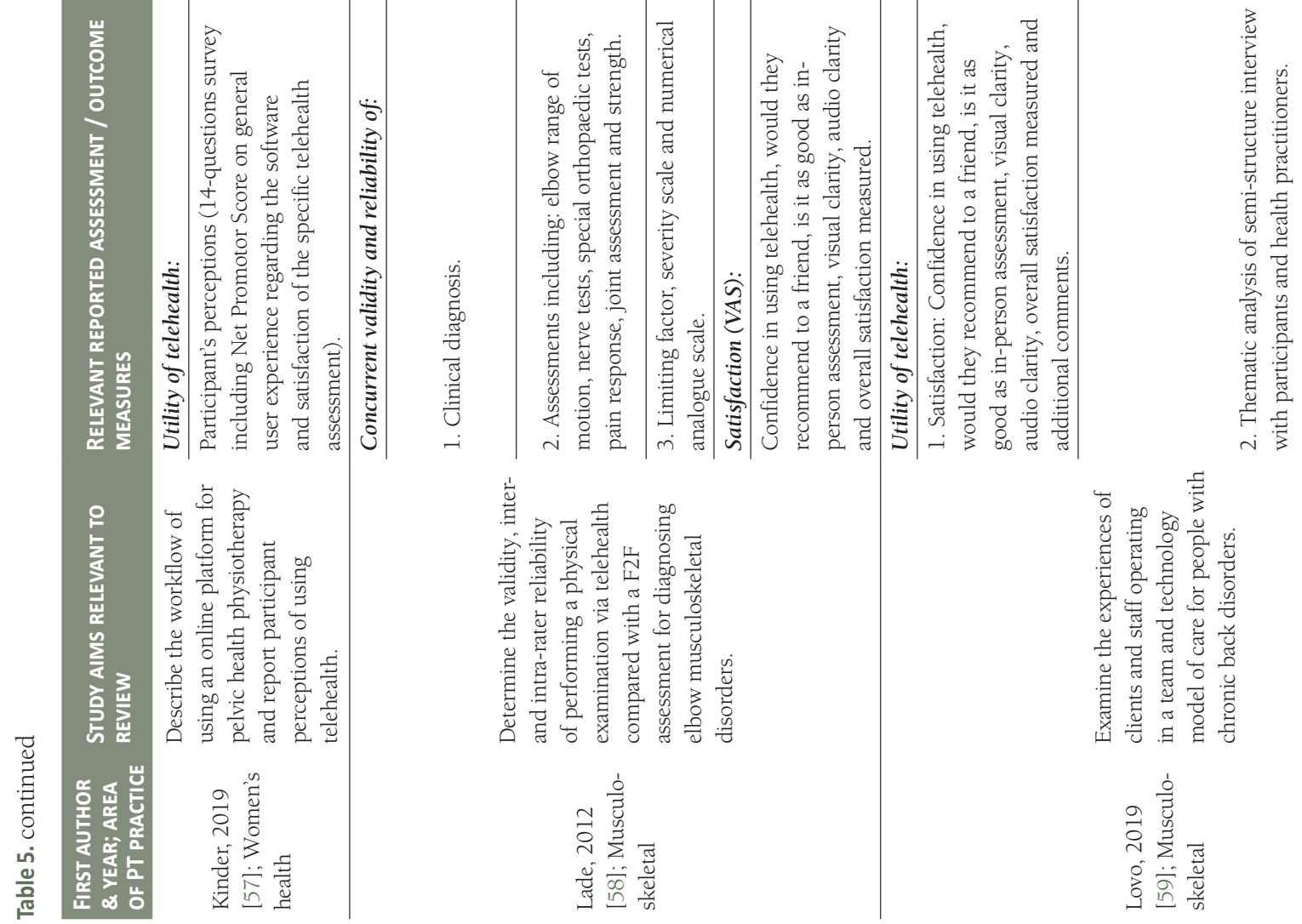

峞

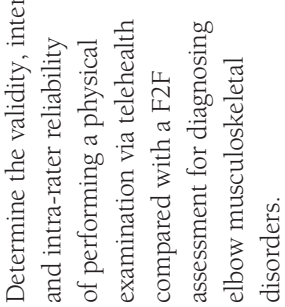

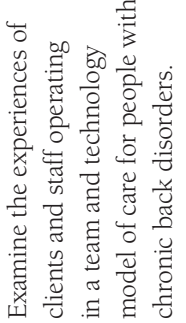

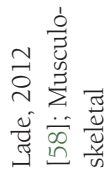




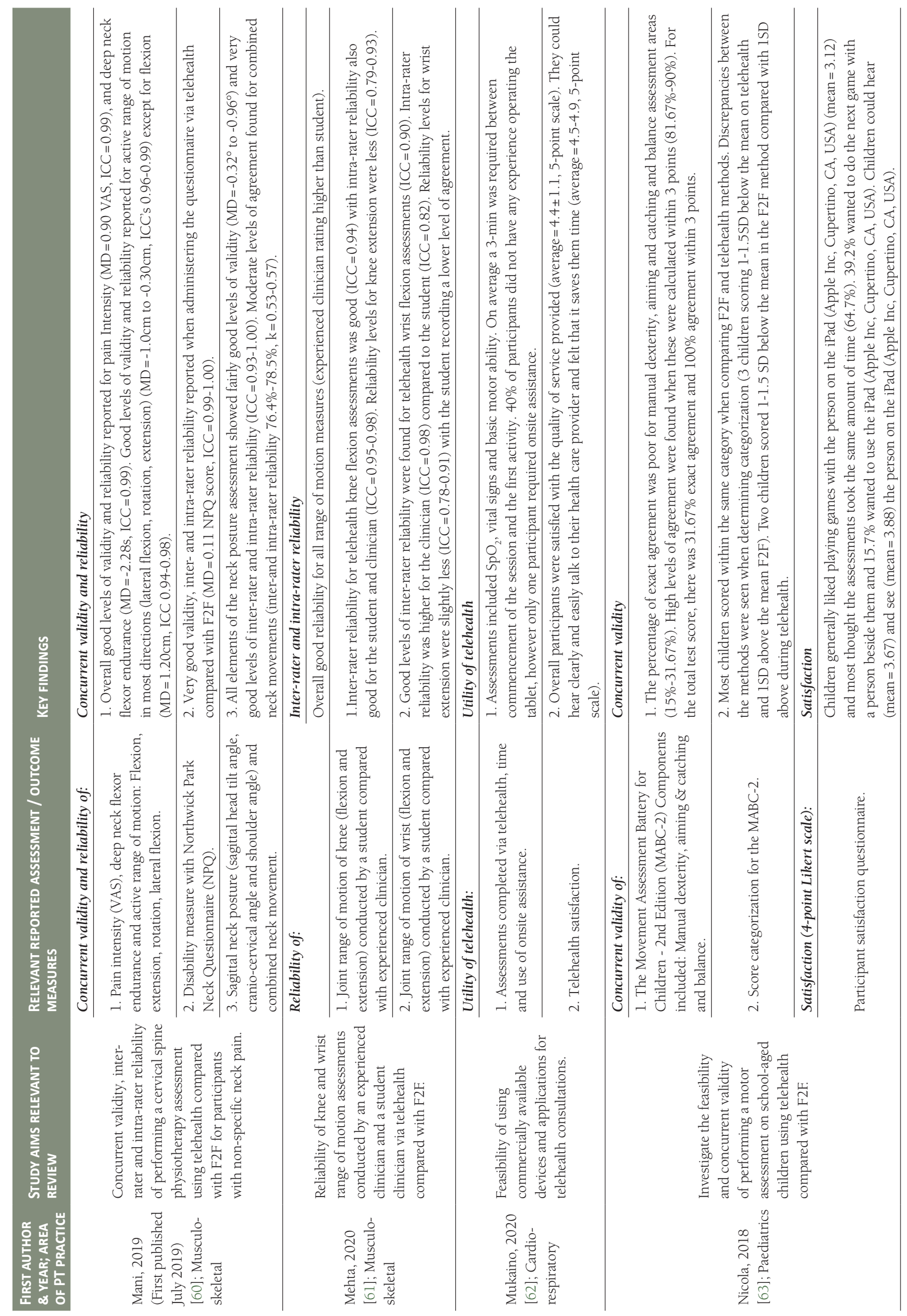




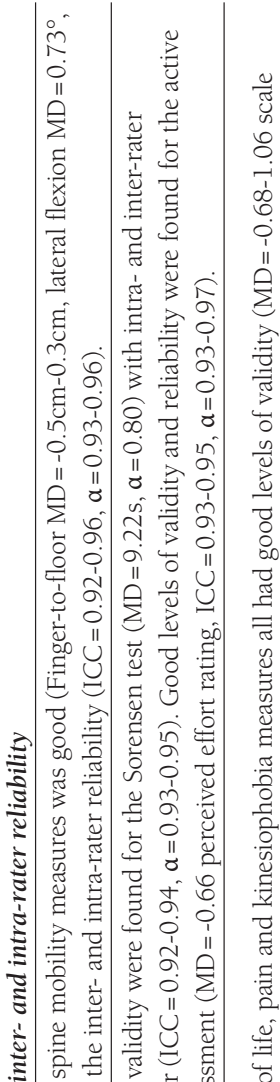

仓ी

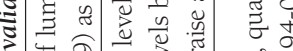

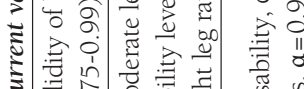

औ

U人
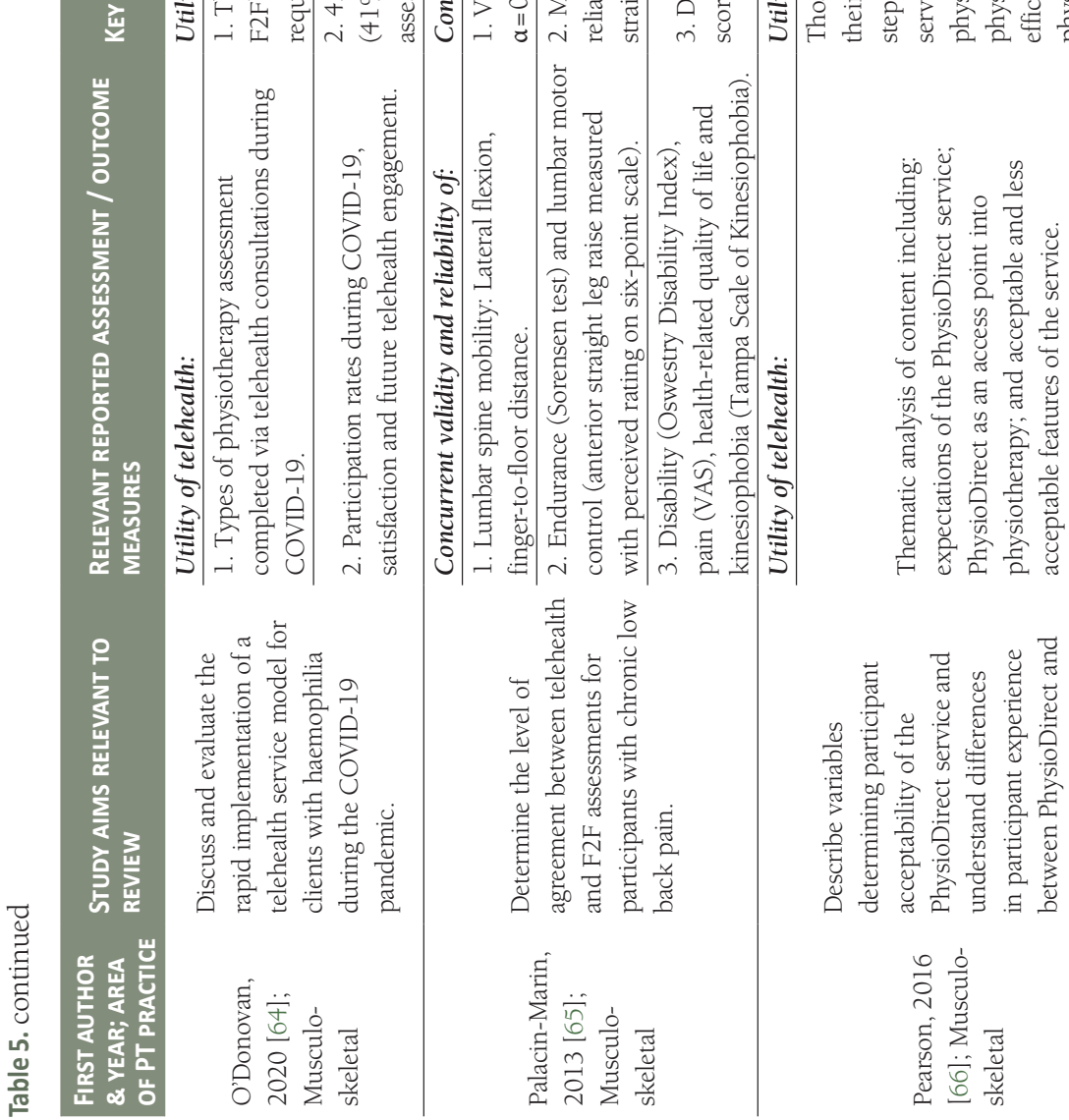

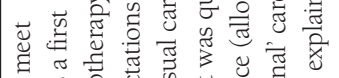

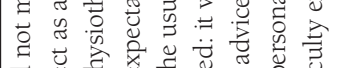

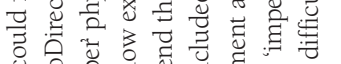

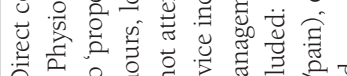

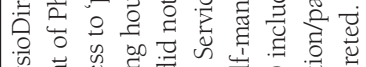

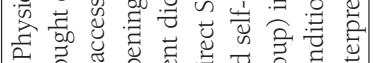

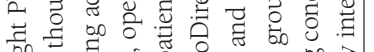

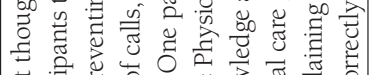

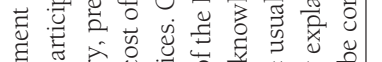

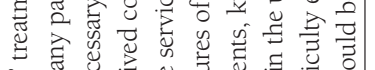

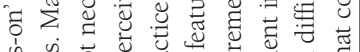

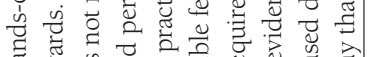

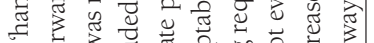
bo

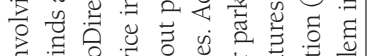

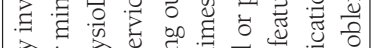

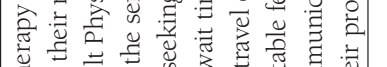

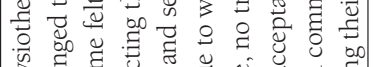

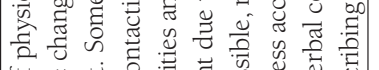

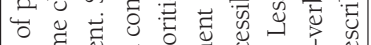
气

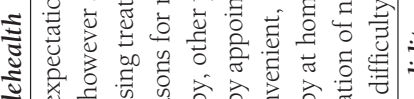

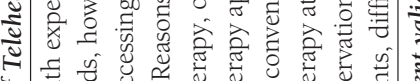

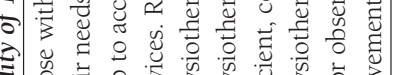

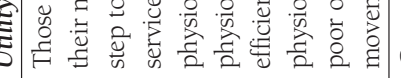
离

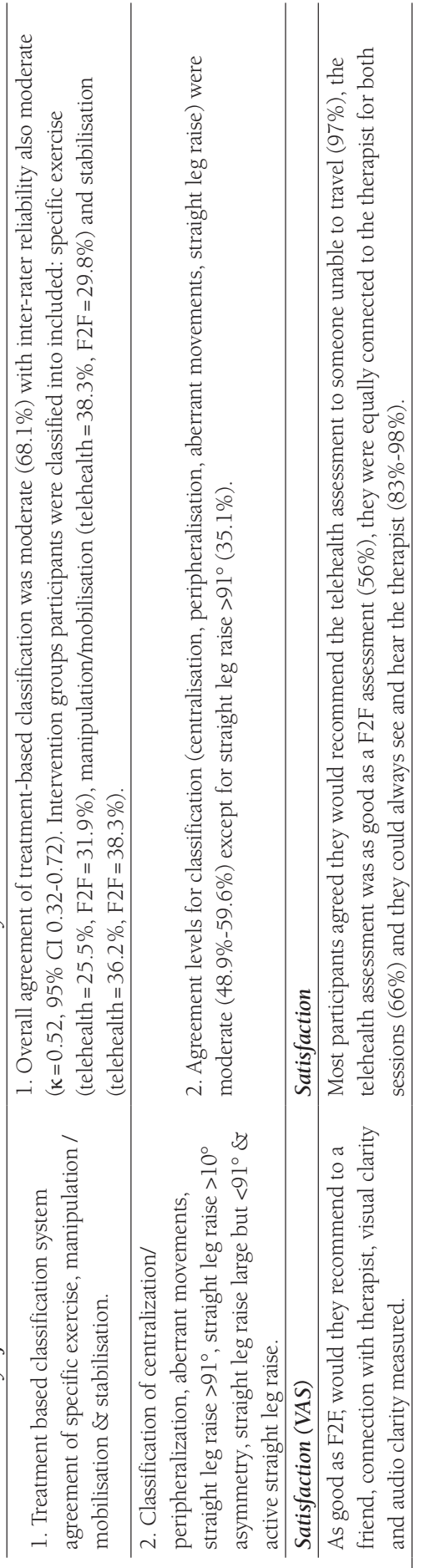

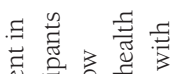

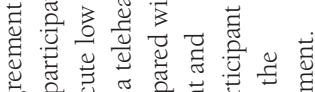

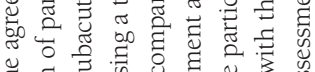

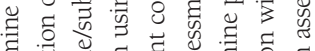

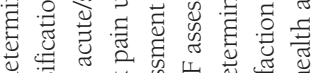

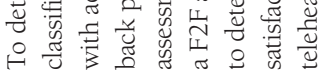

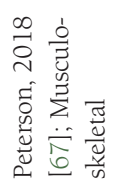




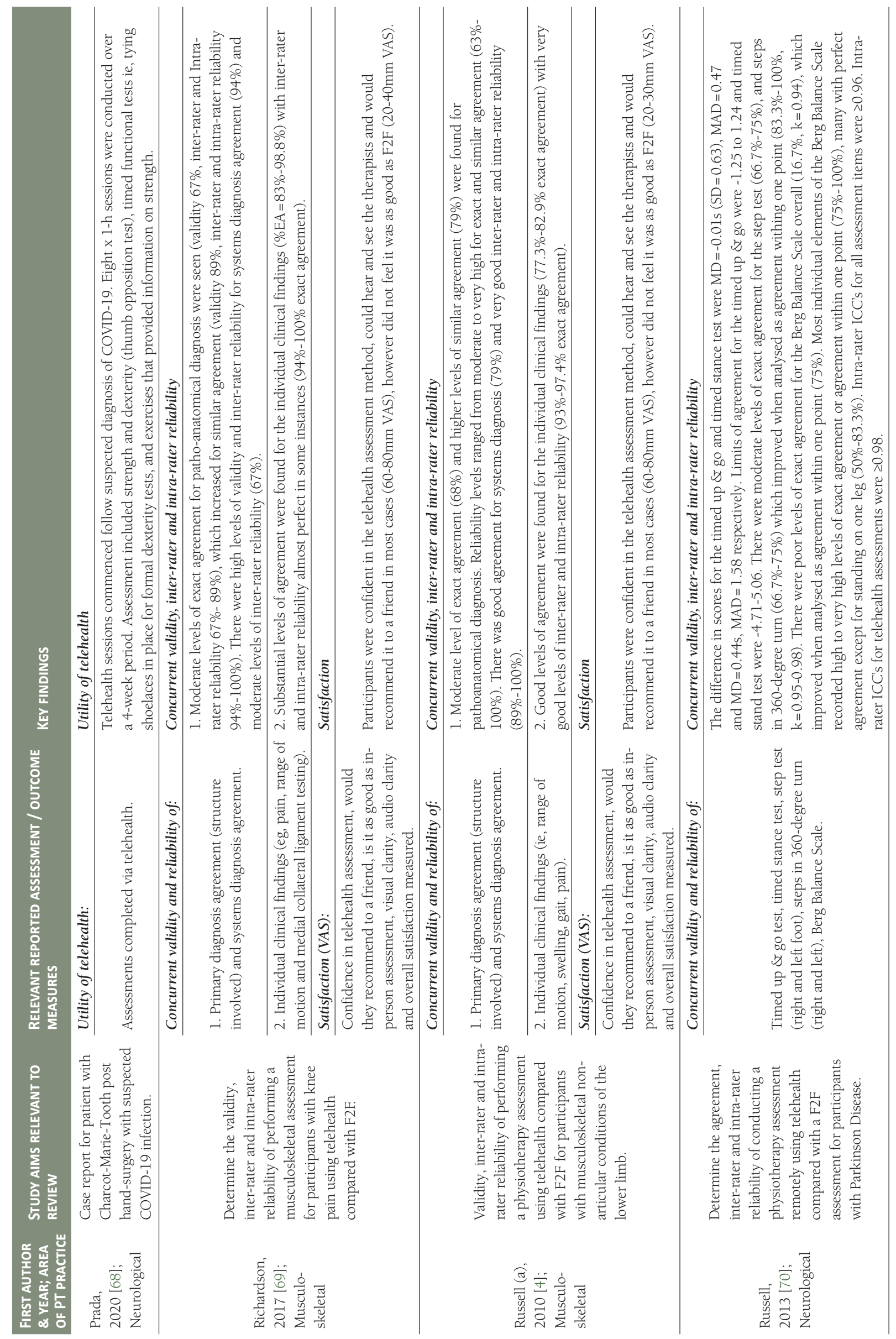



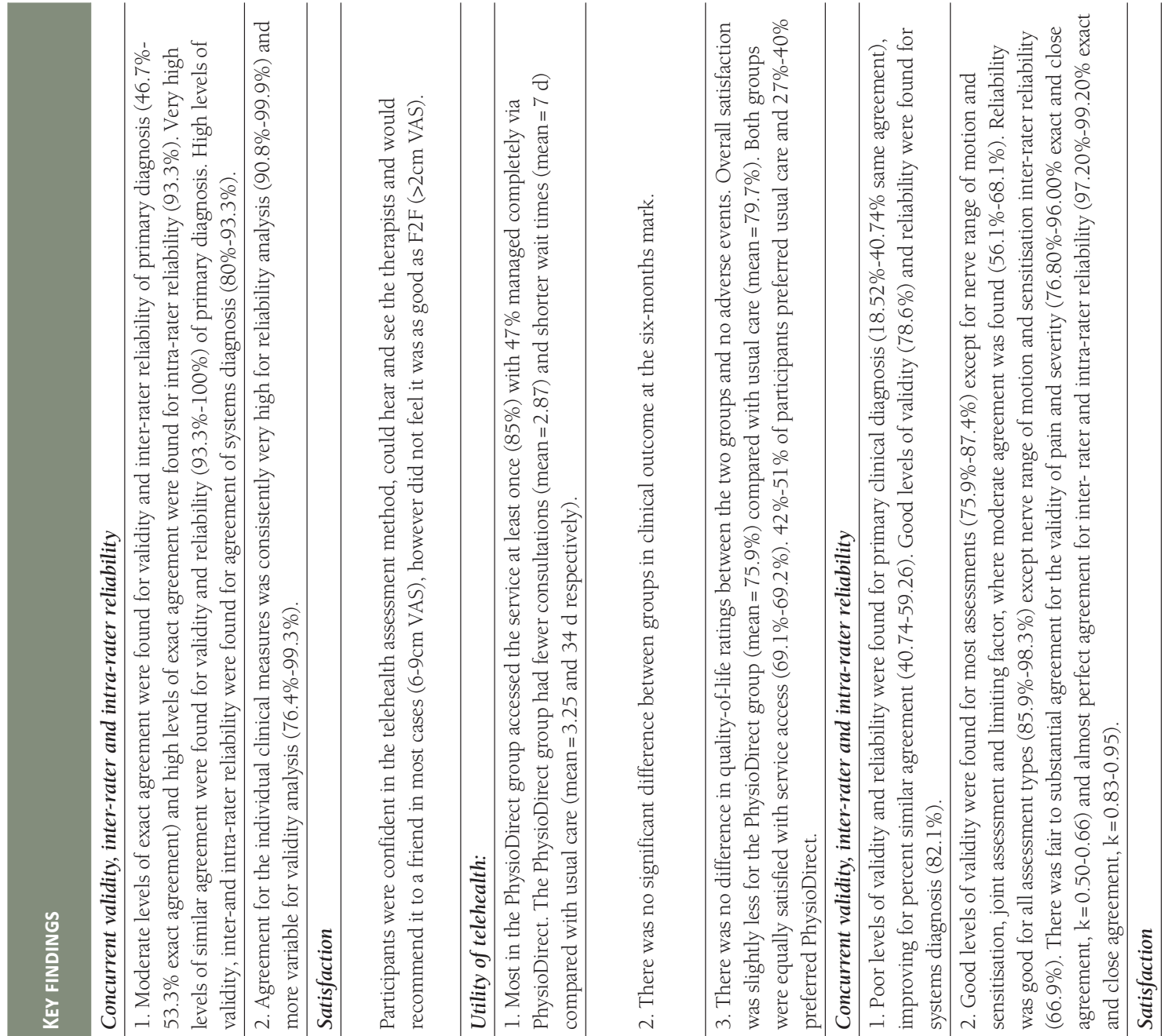

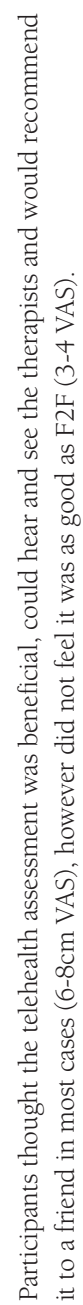
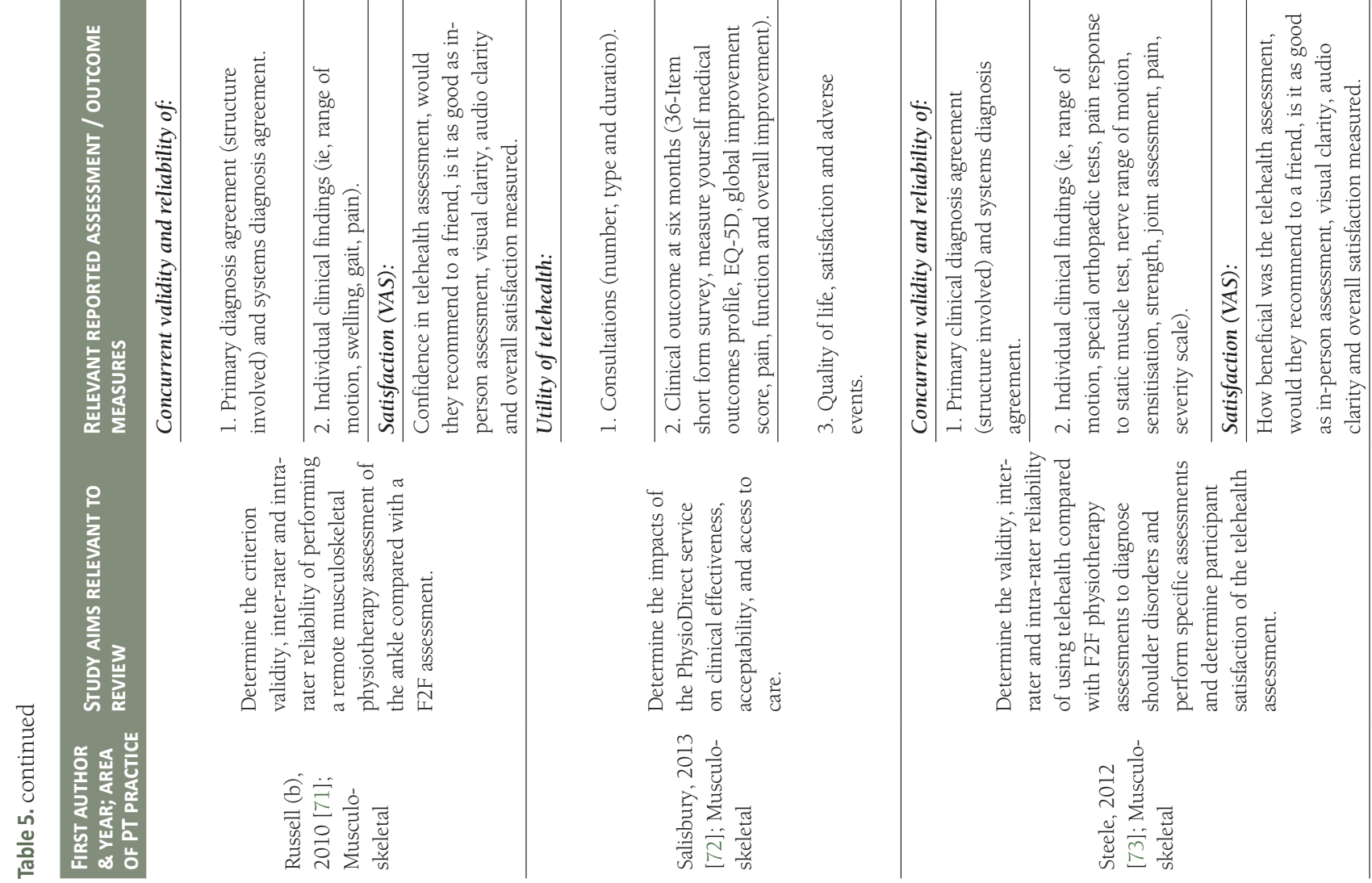

옹ํ․

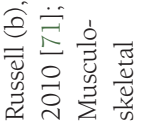

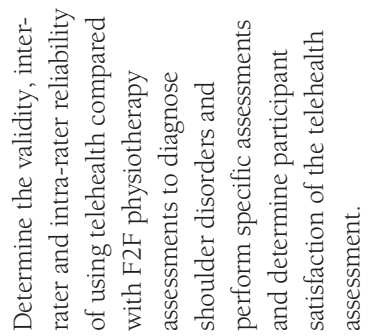

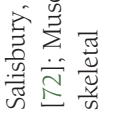

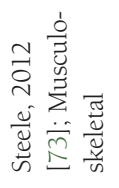




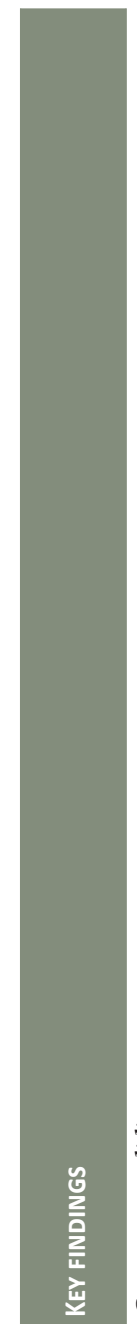

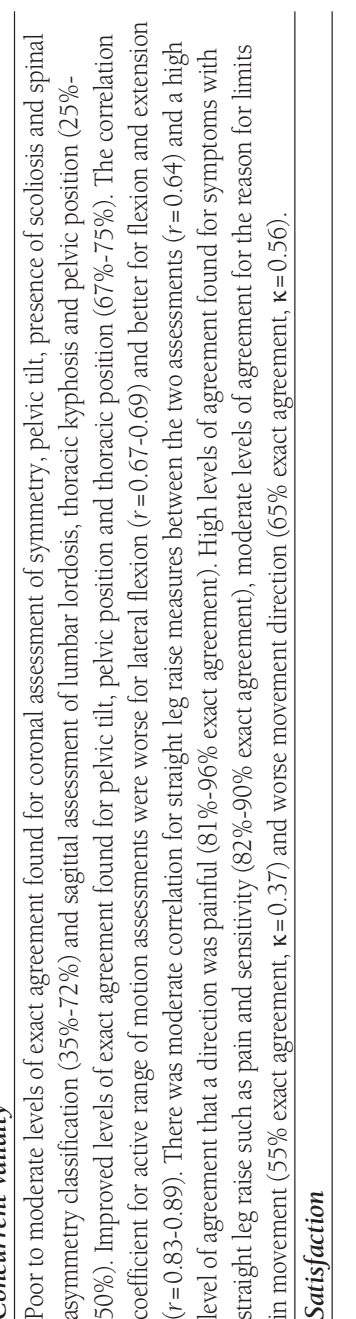

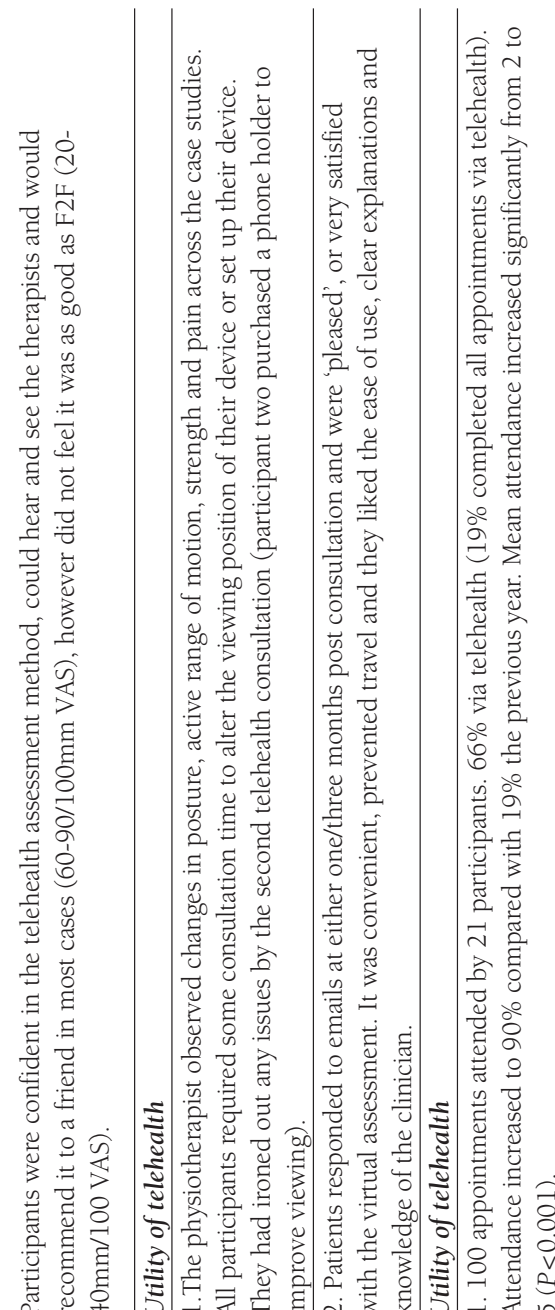

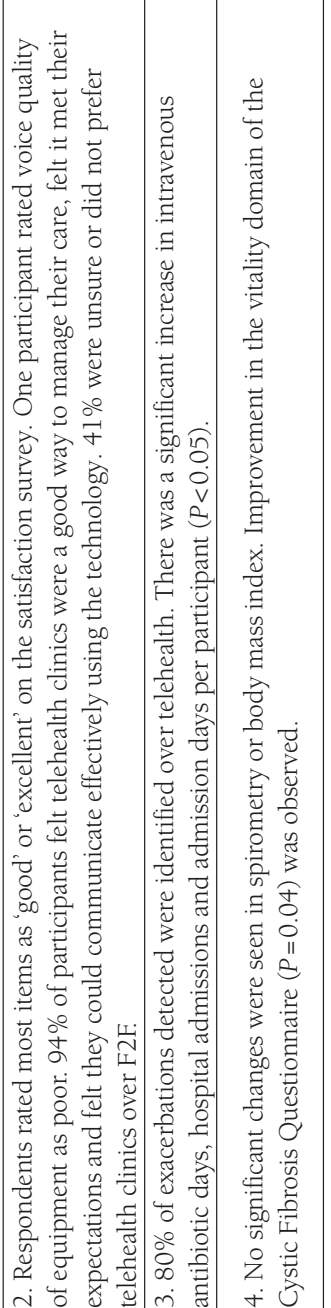

चี

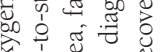

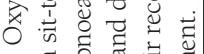

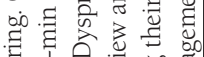

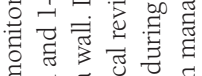

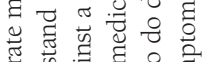
एँ

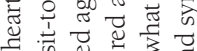
돈.

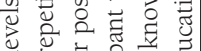

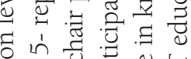

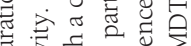

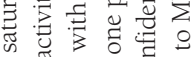
表 诺

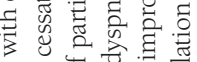

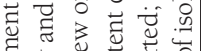

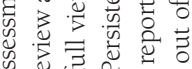

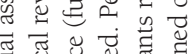

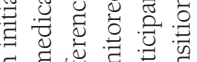

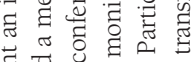
芯芯过苛 ₹

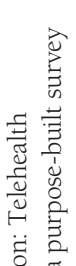

氧

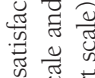

芯

菏

戈节
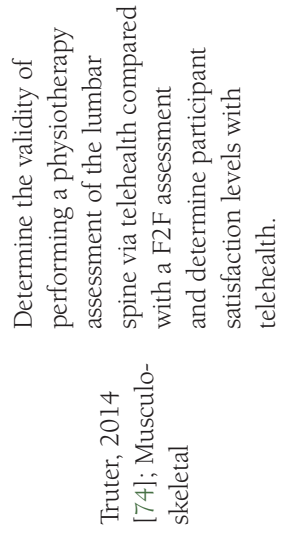

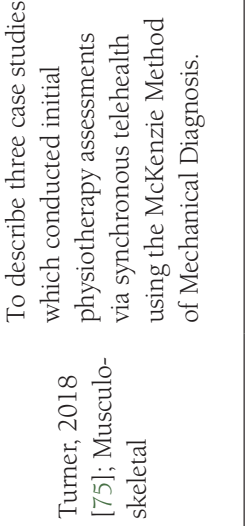

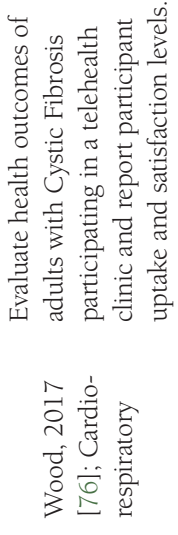

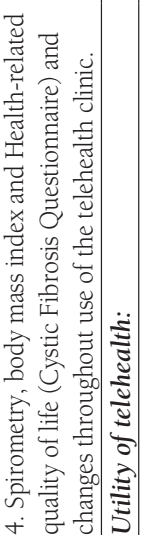

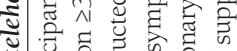

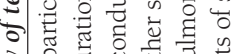

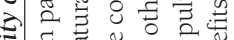

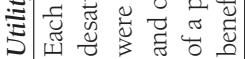

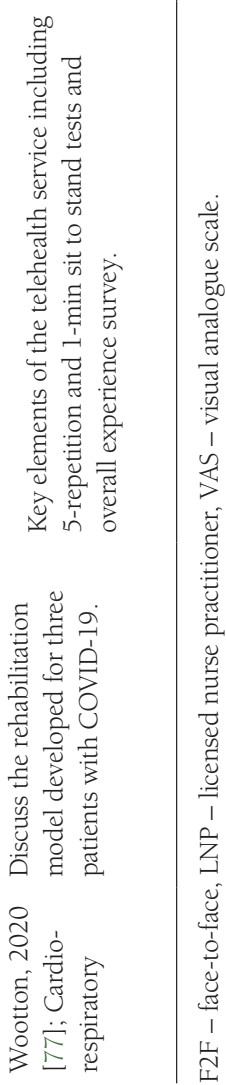




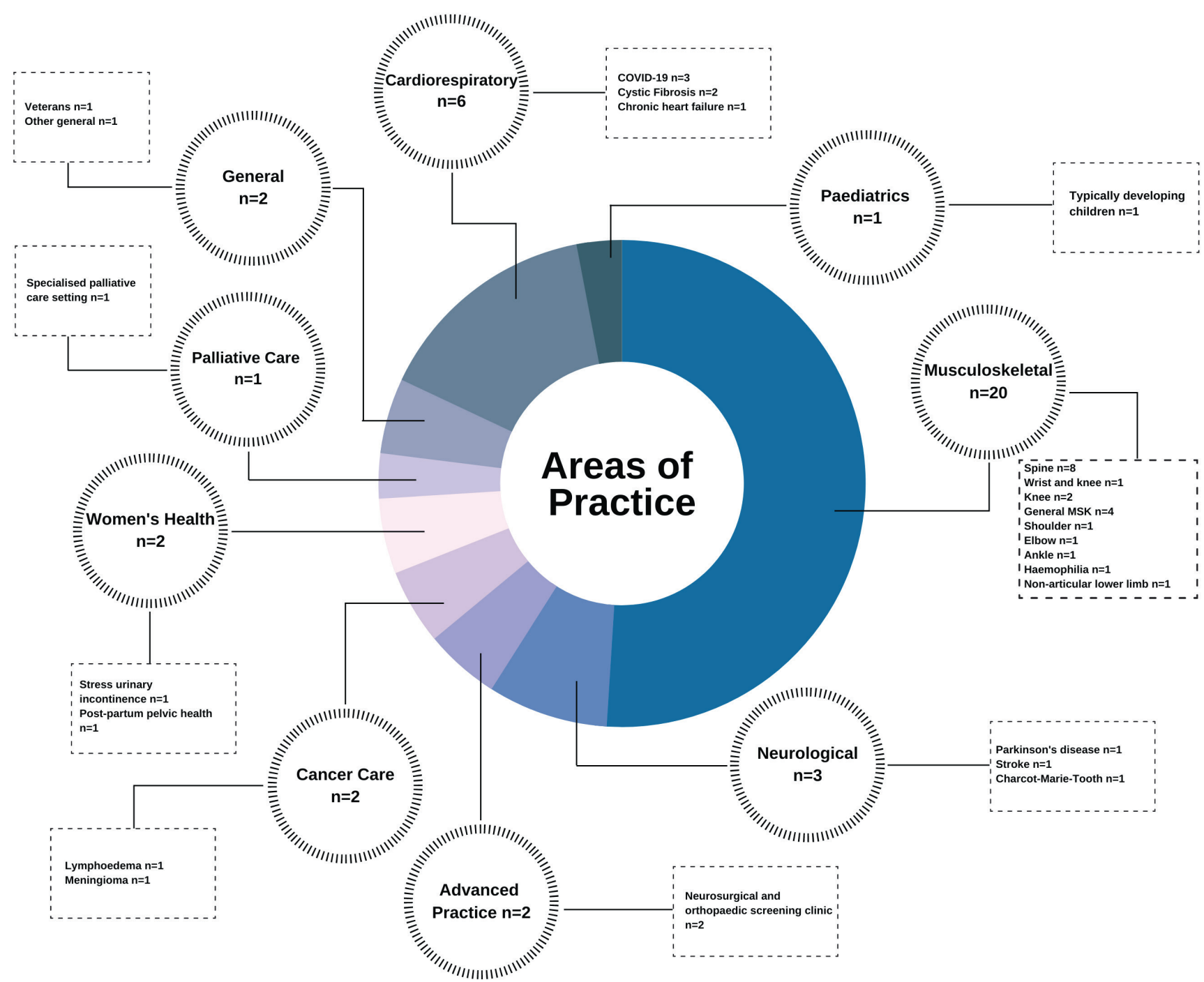

Figure 2. Physiotherapy practice areas of included studies and breakdown of population types for those studies with the largest representation.

to pain, administrative issues, and actions, ie, bookings and advice on physical activity [48]. The value of interprofessional practice, including ability to mentor, train and provide patient-centred care was recognised, along with the benefits of decreased travel time and childcare requirements [53].

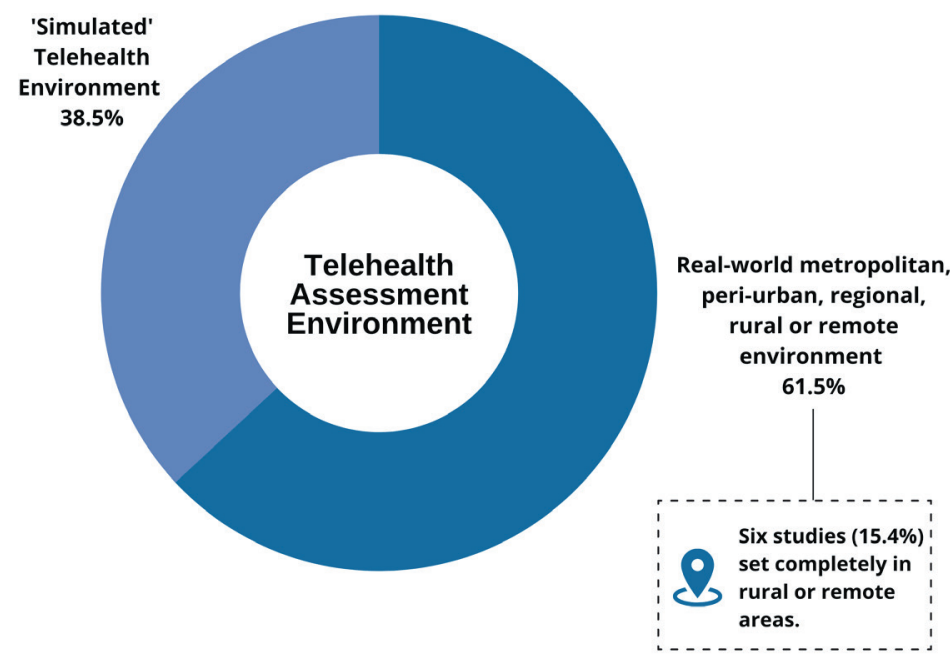

Figure 3. Environments where telehealth assessments were conducted.
Taken together, these studies indicate some feasibility of using synchronous physiotherapy telehealth assessments in a range of areas of physiotherapy, suggesting it can provide benefits to the community and help to maintain or establish service provision when physiotherapy presence or expertise is lacking or when disease transmission is of concern.

Utility of telehealth assessments in rural and remote environments

Telehealth assessments occurring in rural and remote areas (Figure 3) were offered because physiotherapy services in the region were lacking or participants required consultation with an experienced physiotherapist in a specialised area of practice. These studies were conducted in various countries throughout the world with participants including those with cystic 
fibrosis (CF) [76], neurosurgical and/or orthopaedic concerns [46], chronic back disorders [53,59], stress urinary incontinence [44] or veterans with varying concerns [10]. Comparisons between synchronous telehealth assessments and face-to-face physiotherapy assessments for fly-in, fly-out services [46] or specialist centre appointments [76] were investigated. Two studies used nurse practitioners as assistants $[53,59]$ with one of these also using a remote presence robot (RPR) (51) to perform the remote physiotherapy assessment. These studies reported a variety of findings which included: offering a telehealth clinic increased attendance rates [76], participants felt satisfied $[44,53,59,76]$ and confident [59] with the telehealth assessment most of the time, expectations of the assessment were met via telehealth, and that it appears to be cost-saving when compared with a fly-in, fly-out service [46].

Key themes from patients and health care providers using telehealth consultations across rural areas included: that telehealth enhanced access to care (particularly for those in underserved areas), was more convenient, promoted interprofessional communication and team functioning $[10,59]$ and appeared safe with no reports of safety incidents [46]. Having a licensed nurse practitioner on site during the telehealth physiotherapy assessment allowed for identification of unexpected issues, was capacity building and helped improve holistic care of individuals $[10,59]$. Challenges identified by those participating in telehealth consultations in rural areas included: the consent procedures look longer; a lack of access to equipment/specialised equipment in the home setting; using unfamiliar technology; viewing and positioning of the camera; poor quality video; and problems with data file size [10].

\section{Satisfaction and acceptance}

Client and health care practitioner perceptions regarding acceptability and/or satisfaction with physiotherapy telehealth assessments were reported in 19 of the included studies, with most stating they were satisfied with the telehealth assessment and consultation process. Nine studies conducted similar satisfaction surveys $[4,45,58,59,67,69,71,73,74]$, with questions asked rated on a visual analogue scale (VAS). The questions included: 1) level of confidence in, or how beneficial they rated the online assessment, 2) would they recommend a telehealth assessment to a friend who is unable to travel, 3) is telehealth as good as face-to-face appointments, 4) visual clarity, 5) audio clarity, and 6) overall satisfaction $[4,45,58,67,69,71,73,74]$. Most questions asked rated highly in each of these nine studies ( $>6 / 10 \mathrm{VAS}$ ). If given the choice, many preferred the face-to-face method of assessment. This trend was also seen in another study [76], indicating high levels of agreement that the timing, organisation, communication, and overall expectations were met using this service delivery method, but most preferred face-to-face appointments if available.

A participant satisfaction questionnaire specifically developed using age-appropriate language was completed with children who participated in the feasibility and validity study of telehealth physiotherapy services conducted by Nicola and colleagues [63]. Participants appeared to like using the iPad (Apple Inc, Cupertino, CA, USA) (mean >3), however, only 15.7\% of children would pick 'playing the next game' (assessment) on the iPad (Apple Inc, Cupertino, CA, USA), with 39.2\% wanting to play with a person beside them and $45.1 \%$ happy to do either.

Ratings of participant and/or clinician experience and satisfaction with telehealth physiotherapy assessments during the COVID-19 pandemic were reported in three of the included utility studies in this review $[49,62,64]$. These papers reported overall good levels of satisfaction with telehealth assessments, one reporting no statistically significant difference in satisfaction levels between face-to-face, telehealth or combined types of services and a higher satisfaction level for participants in the telehealth group for appointment scheduling $(P<0.001)$ [49]. One study reported mean overall satisfaction levels of $4.4 \pm 1.1$ on a 5-point Likert scale, reported participant perceptions of better access to health care services using telehealth (mean $=4.9 \pm 0.3$ ), and found participants believed telehealth saved them travel time (mean $=4.9 \pm 0.3$ ) and allowed them to easily talk to their health care provider (mean $=5$ ) [62]. The third study reported that participants with haemophillia were interested' or 'very interested' in participating in a remote annual physiotherapy assessment, with $41 \%$ of participants satisfied and 53\% very satisfied with their telehealth consultation [64].

Healthcare provider and participant acceptance was reported to varying degrees across three of the studies investigating the PhysioDirect (PD) telehealth service compared with usual care (UC) in the United Kingdom (UK) $[54,66,72] .44 \%$ of physiotherapists working in the PD telehealth service and 50\% of physiotherapists working outside of the PD telehealth service disagreed/strongly disagreed that many physiotherapy problems can be managed without ever having to see a physiotherapist face-to-face. However, over $73 \%$ of physiotherapists agreed that clients would be better off speaking with a physiotherapist over the phone rather than waiting for a face-to-face appointment, with many reporting they would encourage a family/friend with a physiother- 
apy related issue to contact PD services in the first instance [54]. UC physiotherapy consultations rated significantly higher in terms of satisfaction when compared to telehealth [72] and the acceptance of a telephone physiotherapy consultation service was variable, with some participants commenting on the 'hands on' nature of physiotherapy not able to be accomplished over the phone and that the PD service impaired access to 'proper physiotherapy services [66]. The features of the PD service that appeared to be acceptable to participants included convenience, access to a helpful and knowledgeable physiotherapist and that it fostered delivery of self-management advice. The PD service was reported by some as 'impersonal', with communication difficulties impacting service quality [66].

\section{Technology and usability}

The video conferencing and telephone-based technologies used to perform telehealth assessments are listed in Table 4. The most common were telephone telehealth systems (used in ten studies) and the 'eHAB telerehabilitation system' (NeoRehab, Brisbane, Australia) which was used in nine. A variety of laptop and desktop computer systems was used, along with wall-mounted, external or embedded cameras. Some papers required participants to use their own smart device (ie, iPad, tablet, phone) during the telehealth consultations, whilst others had telehealth assessment equipment set up in a room ready for the consultation. Additional peripherals such as pulse oximeters, LCD screens, tripods, portable speakers and microphones were used in various studies. Several studies reported inconsistencies in technology as limitations, which included: low/fading audio and sound quality [47,56,58,70]; voice disconnection [60]; problems with video quality [56,58]; communication failures [69]; image resolution issues [74]; freezing of live images [60,71] and other technical problems not specified [52]. The usability of telehealth to complete physiotherapy assessments was reported in two studies $[47,56]$ which used the System Usability Scale [78] as an outcome measure. Both studies reported positive responses (scores $\geq 85$ out of a possible 100) and patients seemingly willing to adopt the telehealth technology.

\section{Challenges encountered during telehealth assessments}

Although there were reports that participants 'embraced' the technology used in the telehealth assessments [59], several challenges impacted the effectiveness of telehealth assessments in the included studies. Teaching participants to accurately perform and report some assessment items, for example orthopaedic tests, was challenging [71]. Communication was also challenging, as those participants less experienced in video conferencing etiquette appeared more reserved in their conversation as they attempted to take in information. Some participants had more difficulty explaining and comprehending information [71] or did not stay in the range of the microphone. Others did not wish to de-robe during the telehealth consultation for a variety of reasons, including cultural reasons and this may have impacted upon the visibility of posture and results of range of movement assessments [74]

\section{Economic impact}

The economic impact of utilising telehealth assessments in place of face-to-face assessments was investigated in two utility studies included in this review. One study involved a cost audit comparing a video conference telehealth physiotherapy service with a fly-in, fly-out physiotherapy service [46]. Telehealth appeared to be a cost-effective option for rural services, with an estimated 12-month cost of AUD\$66518.00 compared to AUD $\$ 76384.00$ for a fly-in, fly-out service [46]. The other study compared cost-effectiveness of a telephone-based telehealth physiotherapy service (PD) with usual face-to-face care (UC) [55]. The authors found the difference between the PD and UC groups was minimal in terms of cost, finding PD is probably more cost-effective if the physiotherapist's time is productive. However, underutilisation of the service increased costs of the PD service slightly compared with UC, which were then offset by improvements identified through health-related quality of life questionnaires (quality adjusted life years).

\section{Validity and reliability of telehealth assessments}

The assessment tools investigated across the validity and reliability studies included in this review and the results of those studies are summarised below, with details provided in Table 5. The types of assessments completed by physiotherapists via telehealth were either pre-determined or decided upon by the assessing clinician at the time of the assessment. Such discretion was apparent in six of the validity and/or reliability studies $[4,45,58,69,71,73]$, where telehealth clinicians could decide which assessment measures to use. These studies all reported on the validity and reliability of clinical diagnosis (primary or pathoanatomical, and systems diagnosis) and management decisions rather than specific assessment tools, except for one study [58] which reported on both. 


\section{Validity of telehealth assessment}

Assessments of the upper limb performed using synchronous forms of telehealth that were associated with good or very good levels of concurrent validity when compared to a face-to-face assessment were (Table 5): assessments for participants with elbow [58] or shoulder [73] pathology inclusive of range of motion (ROM), special orthopaedic tests, pain response and strength (75\%-90\% agreement); diagnosis and management decisions of shoulder pathology (83.3\% exact agreement) [45]; pathoanatomical diagnosis of the elbow (73\% similar agreement) [58]; systems diagnosis of shoulder and elbow pathology (73\%-78.6\% agreement) [58,73]; and circumferential measures taken by a caregiver via telehealth under the direction of a physiotherapist to determine total arm volume for patients following breast surgery (largest mean difference occurring in circumferential measures taken $10 \mathrm{~cm}$ below the elbow, $\mathrm{MD}=0.28 \mathrm{~cm}-0.30 \mathrm{~cm}$ ) [52].

Synchronous telehealth physiotherapy assessments of the lower limb associated with good or very good levels of concurrent validity when compared to a face-to-face assessment included (Table 5): clinical diagnosis of knee and ankle pathologies (exact or similar agreement in 83.3\% - 93\% of cases) [45,69,71]; systems diagnosis for knee or ankle pathologies ( $\geq 80 \%$ observed agreement) [69,71]; and primary clinical diagnosis ( $79 \%$ similar agreement) and systems diagnosis (79\% observed agreement) of non-articular lower limb injuries [4]. Good levels of concurrent validity were also found for assessments of the cervical and lumbar spine, including (Table 5): ROM [60,65,74]; active straight leg raise [65]; lumbar spine assessment of painful direction of movement [65]; neck pain intensity [60]; deep neck flexor endurance [60]; cervical spine posture [60]; questionnaires to determine levels of disability, quality of life and pain [65]; and diagnosis and management decisions (including decisions about necessary investigations) for pathologies resulting in lumbar spine symptoms (83.3\% exact agreement) [45].

Assessments that required observation and/or timing, such as the timed-up-and-go test (TUG), timed stance test, step test and steps in 360-degree turn, also showed good levels of concurrent validity when compared with face-to-face assessments [47,56,70], as did the six-minute walk test (6MWT) and grip strength test, which were both reported as possessing concurrent validity when performed via telehealth in participants with chronic heart failure [56] (Table 5).

Some assessments conducted using synchronous telehealth methods were standardised physiotherapy assessments such as The Movement Assessment Battery for Children (2 ${ }^{\text {nd }}$ edition) (MABC-2) assessment conducted in typically developing children [63], the ABILHAND assessment conducted in participants following stroke [41] and the Berg Balance Scale for participants with Parkinson disease [70]. These assessments were found to have higher levels of concurrent validity when compared to face-to-face assessment if scores were either analysed within a certain number of points, or totaled. The level of validity decreased when looking at exact agreement or individual items within each assessment tool, such as standing on one leg (50\% exact agreement) or threading a needle and fastening a snap ( $\mathrm{k}$ coefficients 0.15 and 0.20 respectively).

Moderate to good levels of exact agreement were found for primary clinical diagnosis and systems diagnosis of non-articular lower limb injuries [4]. Moderate levels of concurrent validity were also found for cervical flexion and combined movements of the cervical spine [60]; and overall pathology classification decisions made by assessors (68.1\%) for participants with low back pain [67], with a significant difference found between the telehealth and face-to-face assessments for straight leg raise classification greater than 91 [67].

Poor to moderate levels of concurrent validity were found for upper limb nerve testing and sensitisation assessment [58,73], joint assessment of the elbow and shoulder (46\%-64.4\% agreement) [58,73], pathoanatomical diagnosis of the shoulder (59.72\%) [73], the Sorenson test [65], and postural assessments investigating symmetry, pelvic tilt and spinal asymmetry in the coronal plane [74]. Additional details are provided in Table 5.

\section{Inter-rater reliability in telehealth assessment}

Good to very good interrater reliability was reported for synchronous telehealth assessments involving the upper limb, including (Table 5): elbow and shoulder ROM; upper limb special orthopaedic tests; strength assessments; elbow and shoulder joint assessments; pain; and pain response to static muscle tests (87\%-98.3\% agreement) $[58,73]$. Lymphoedema assessment measures of the upper limb such as arm circumferential measures and calculations of total volume were reported to have high levels of inter-rater reliability (ICC 0.810.98) [52]. Good to very good levels of inter-rater reliability were found for lower limb assessments, including: knee ROM [61]; knee swelling [43]; and diagnosis of knee pathology [69], non-articular lower limb injuries [4] and ankle disorders [74]. Assessments of the cervical and lumbar spine [60,65], including ROM, lumbar 
spine mobility, lumbar spine motor control, posture, and muscular endurance, also showed good to very good inter-rater reliability (ICC $0.93-0.99$ ). Assessments such as functional strength testing (30-second chair-tostand test), TUG, and gait assessment (Tinetti test) displayed good inter-rater reliability $(0.79 \leq \alpha \geq 0.87)$ [43], as did the 6MWT, timed stance test, step test, steps in 360-degrees turn, grip strength test, and Berg Balance Scale (all ICC >0.96, $\alpha=0.76$ for Berg Balance Scale) $[43,56,70]$.

Moderate to good levels of inter-rater reliability were reported for primary diagnosis of upper limb disorders (73\% agreement elbow and shoulder) $[58,73]$ and assessing the cervical spine using combined neck movements (78.5\% agreement) [60].

Poor to moderate levels of interrater reliability were reported for upper limb nerve tests involving nerve ROM or assessment of neural sensitisation (66.9\%-68\% agreement), and systems diagnosis of elbow disorders (64\% agreement, $P=0.11)[58,73]$. Inter-rater reliability for the assessment of scars was poor $(\boldsymbol{\alpha}=0.34)$ with authors suggesting that still photographs may be better than synchronous telehealth via video conference [43].

\section{Intra-rater reliability in telehealth assessment}

Good to very good rates of intra-rater reliability were reported for assessment of the elbow (81\%-98\% agreement) [58], shoulder (\% agreement >85\%) [73], cervical spine (ICC>0.93) [60], and lumbar spine using ROM, endurance and motor control assessments (ICC>0.94) [65]. Very good levels of intra-rater reliability were found for the diagnosis of knee pathology [69], non-articular lower limb injuries [4], ankle disorders [71], elbow [58] and shoulder disorders [73]. Very good levels of intra-rater reliability were reported for knee and wrist flexion/extension ROM assessments conducted by experienced clinicians with healthy participants, however levels decreased when student physiotherapists performed the same measures [56,61]. Intra-rater agreement was high for the TUG, 6MWT, timed stance test, step test, steps in 360-degrees turn, grip strength test and Berg Balance Scale (ICC $\geq 0.96)$ [70].

\section{DISCUSSION}

The review identified a total of 39 studies published over the past decade which have investigated the validity, reliability or broader utility of using synchronous telehealth physiotherapy assessment methods across MSK, neurological, cardiorespiratory, paediatric, women's health, cancer care, palliative care, advanced practice and general fields of physiotherapy practice. Just over $60 \%$ of studies published were in a real-life community, hospital or home setting, with six of these papers set in a rural or remote area that faced limitations in access to physiotherapy services. The major findings of this review demonstrate that performing many types of physiotherapy assessments via synchronous forms of telehealth appears to be valid and reliable, with those more observational in nature more easily performed and having higher levels of validity and reliability. Participant satisfaction levels with telehealth assessments were generally high, although when asking participants if they would favour or select a telehealth assessment compared with a face-to-face assessment, most would prefer the latter. The utility of telehealth amongst physiotherapy areas of practice is expanding, with increased perceptions of its acceptability among health care professionals and recipients of care when service access is a problem. Research within this area is also growing, with approximately two thirds of included studies published in the last 5 years, and later studies adapting their telehealth assessment techniques based on the findings and issues reported by earlier researchers.

\section{Areas of practice}

To our knowledge, this is the first systematic review that has investigated the validity, reliability and broader utility of performing physiotherapy assessments using synchronous forms of telehealth, across all areas of physiotherapy practice. The current review considered a broader range of physiotherapy assessment tools than have been previously considered. For example, a systematic review investigating the validity and intra-and inter-rater reliability of telerehabilitation (TR) physiotherapy assessments for patients with specific musculoskeletal disorders was reported in 2016. That review, conducted by Mani and colleagues [19] identified eleven studies that met eligibility criteria and included both synchronous and asynchronous forms of assessment. A more recent review by Grona and colleagues [25], also in the field of musculoskeletal physiotherapy, investigated video conferencing consultations, including both assessment and intervention aspects of the telehealth service. Seven of the studies that were included in both of these previous systematic reviews also met the eligibility criteria of our systematic review. Neither of these previous systematic reviews investigated all synchronous forms of physiotherapy assessments used across all areas of physiotherapy practice. 
Many areas of physiotherapy clinical practice do not have enough presence in the literature to enable accurate determination of the validity and reliability of relevant synchronous telehealth assessments. More than half of the papers in this review focused on assessments used in the field of musculoskeletal physiotherapy. To address issues of access through approaches that include telehealth assessment, it is important to investigate the clinometric properties of assessment tools used commonly in all areas of physiotherapy practice. The results of our review show that several areas of physiotherapy practice are completely lacking in research regarding the validity, reliability and utility of telehealth assessments. There were no studies meeting our inclusion criteria that investigated physiotherapy assessments with infants or with children of any age with existing difficulties in gross motor function. This population arguably would benefit greatly from telehealth assessments performed within the home setting, not only to ease the travel burden for families and improve access, but also to gain a better insight into the child's function in the home environment. Children feel more comfortable and display a greater repertoire of skills when in their home environment, as compared to that of a physiotherapy clinic [79]. There were also no studies that examined the validity and reliability of synchronous telehealth assessments in the clinical areas of elite sport, gerontology, occupational health, men's health or other specialty areas of physiotherapy practice, including hand and orofacial physiotherapy. Only a very small number of validity and reliability studies, investigating assessments relevant to limited types of health conditions, were identified in cardiorespiratory, paediatrics, chronic pain, neurological and cancer care (particularly lymphoedema) physiotherapy. It could be argued that individuals requiring physiotherapy consultation in these less represented fields of physiotherapy may benefit the most from having valid and reliable telehealth assessments, given the need for specialised services (which may not be present in regional, rural or remote areas) or limited ability to travel due to illness. Individuals requiring a physiotherapy assessment for conditions that fit within these areas of practice (for example, those that have existing cardiorespiratory conditions, are immunocompromised, or older adults), are also those classified in the 'highest risk' groups for COVID-19 and would at times require telehealth rather than face-to-face consultations, particularly when disease transmission is of concern [80]. More comprehensive research in these areas of physiotherapy practice is required.

\section{Telehealth assessment environments}

Many validity and reliability studies included in this review were set in a 'simulated' telehealth environment and therefore aspects of their findings require cautious interpretation. The environment in which telehealth assessments are performed can impact the assessment quality, with aspects such as lighting, image resolution and internet speed important for visualisation using synchronous video-based telehealth assessment. It may also influence participants' ratings of satisfaction and acceptance of the method of assessment, as access to technologies and knowledge that a clinician is close-by in a simulated environment could be reassuring.

Some of the case studies completed in hospital environments that described changes in practices for consultations during COVID-19 did, however, require a telehealth assessment to occur despite the clinician and participant being collocated in the same building. Due to the infectious nature of the disease, it was important to keep contact between physiotherapist and participant limited to decrease potential virus transmission. In this circumstance, the results of previous studies conducted in 'simulated' environments is very helpful in guiding the set up and execution of these types of telehealth services.

Nevertheless, many of the utility studies included in this review were conducted in 'real-life' environments where the participant was located in an environment representative of what would occur outside research.

\section{Utility of telehealth assessments}

The use of telehealth in the clinical setting has increased dramatically over the past 12 months, with many clinicians compelled to use telehealth in one form or another during the COVID-19 pandemic. Four of the studies included in this review evaluated or described how clinicians had transitioned to telehealth for maintaining physiotherapy services for their patients $[50,64,68,77]$. Prior to COVID -19 , the use of telehealth was seen as a way to reduce barriers to accessing services, such as distance, inability to travel, or unavailability of a physiotherapist in the local area [7]. Throughout the COVID-19 pandemic, we have seen the value of telehealth change, with physiotherapists completing synchronous physiotherapy assessments while located within the same hospital as the patient, and at times, just outside a patient's room [50]. This change in the way we utilise telehealth services in the 'real-world' encourages us to consider more closely and perhaps increasingly value findings of studies that have been conducted in 'simulated' environments where the physiotherapist and participant are collocated in the same building. The way in which specific assessments were performed via telehealth also needs to be considered when interpreting the results. Some studies did not include a detailed 
description of how the assessments were performed or adapted to meet telehealth requirements, and others used non-standardised, functional activities to assess underlying impairments.

\section{Utility of telehealth assessments in rural and remote environments}

Six utility studies were conducted with participants located in rural and remote geographical areas, providing insight to the challenges and benefits of telehealth assessment. Due to the relatively small number of studies set in these geographical areas, it is important that we see further research investigating synchronous telehealth physiotherapy assessments set in 'real-life' rural and remote settings. Many other studies were completed in 'real-life' environments but located in more urban and metropolitan areas.

\section{Satisfaction and acceptance}

The level of satisfaction and acceptance of physiotherapy telehealth assessments amongst telehealth users was generally high, with satisfaction measured in several studies, across a range of environments. Most participants thought telehealth would be useful for those unable to travel to or access a suitable physiotherapist in their local area but would not prefer it over a face-to-face appointment. This finding is consistent with previous research investigating patient satisfaction using synchronous and asynchronous forms of telehealth in medicine, which reported that telehealth can provide a high-quality service, increase access to care, decrease travel time and empower patients to manage their chronic conditions, but can be met with resistance from those who have difficulty embracing change [81].

\section{Technology and telehealth usability}

There were several studies in this current review that identified inconsistencies in technology as limitations in physiotherapy telehealth assessment $[47,52,56,58,60,69-71]$. Some users were able to easily troubleshoot these issues or bypassed the synchronous telehealth system, for example by positioning a metronome with the participant rather than the remote physiotherapists when conducting a step test [47] and some provided recommendations for future studies, such as using still images rather than a live video feed to assess scars [43]. As technology advances and becomes more readily available within the community, there appear to be improvements in levels of digital literacy with an increased proportion of the population classified as 'digitally native', having grown up with access to technologies and having subsequent competence in navigating those that are new [82]. This may change the levels of acceptance and usability of technologies such as telehealth in the future and further limit inconsistencies identified in this systematic review. Consequently, it would be advantageous to consider developing further research studies within this topic area with the latest technology, for example 4K HD Cameras, inclusive of larger sample sizes.

\section{Challenges encountered}

Telehealth assessments conducted in the studies included in this review at times required clinicians to alter their assessment methods from those typically used during face-to-face consultations. Self-resisted strength assessments, using furniture to assist with the assessment of the shoulder, and educating and utilising another health care provider to assist with the assessments were all techniques used to facilitate the telehealth assessments. Some studies provided resources to participants prior to the telehealth session, which assisted them to understand the assessment process and technologies used.

Some of the difficulties reported in the included studies centred around communication [71], and specifically the explanation and comprehension of information, for example describing pain behavior and location if non-verbal communication was not able to be observed during telephone consultations $[54,55,66,72]$. Some studies also found that participants were more reserved in their conversation when assessment was conducted over telehealth, or that participants did not understand etiquette required for a video conference consultation [71]. Others reported difficulties in observing posture, as some participants were not comfortable de-robing during the telehealth [74] consultation for a variety of cultural and personal reasons. When working with diverse community groups, Pollard and colleagues [83] suggests we must consider the cultural and ethical needs of individuals, which requires planning, flexibility and sensitivity. As we move forward with utilising synchronous telehealth methods to perform physiotherapy assessments in clinical contexts, we must consider the validity and reliability of the assessment measures used with respect to our clients, and also their presenting condition or diagnosis and contextual factors including the environment they are in and available resources, individual needs and cultural beliefs [83]. These are important elements to consider when deciding if telehealth is appropriate for clients, or if another method of assessment and service delivery is appropriate. 


\section{Economic impact}

There were two studies included in this review that investigated the economic effectiveness of conducting telehealth assessments compared to face-to-face or usual care $[46,55]$. These concluded that telehealth assessments were slightly more cost-effective when compared with face-to-face care, especially when services were fully utilised, and calculations considered the impacts of waiting times and their effects on an individual's quality of life. There have been a number of systematic reviews published that have investigated the cost-effectiveness of telehealth or mobile health interventions across medicine [84,85], and psychiatry [86], with cost-effectiveness varying depending on the service and equipment set-up, reporting methods, patient location, travel requirements and needs of the patient. One study concluded that telehealth is most likely cost-effective for patients located in a rural area who have high travel costs [85], however further rigorous studies are required to determine the economic impact that telehealth can have [86], particularly for physiotherapy services.

\section{Validity and reliability of telehealth assessments}

Eighteen of the studies included within this review investigated the concurrent validity and/or reliability of performing synchronous telehealth physiotherapy assessments. The telehealth assessment tools examined in studies included in the current review appeared to be most valid and reliable if they were observational in nature, with the exception of scar assessments and observations of the spine. Telehealth assessments that required a greater degree of technique were also more complex in nature and more difficult to interpret when conducted by telehealth. For example, MSK special tests and neurodynamic testing were less reliable and valid when completed using telehealth systems than when performed face-to-face. This is not surprising, given the extensive training and experience that physiotherapists possess in manual handling and the value that the sense of 'feel or touch' can add to interpretation of assessments [87]. Those assessments that involved the use of questionnaires also appeared to be more valid and reliable than some other types of assessment, when conducted via telehealth. This finding is in line with a previous systematic review which reported good test-retest reliability for questionnaires measuring shoulder function [88]. This is to be expected, as no hands-on contact is required to administer these assessments.

Although there appeared to be good levels of agreement between telehealth and face-to-face assessment findings for many of the assessment types and diagnoses, the impact of one incorrect diagnosis or non-referral on the patient and their health condition must be considered [46]. Client and family centred decision-making must also be considered, where individuals may choose a telehealth assessment based on convenience or travel cost, despite the challenges telehealth may have. The use of telehealth in the absence of other face-to-face forms of physiotherapy assessment may improve health outcomes for clients, particularly those in rural and remote areas or impacted by an infectious disease, as it may mean they are able to access services otherwise unavailable to them.

\section{Limitations of included studies and the present systematic review}

Several limitations of the included studies in this literature review influence the conclusions we can draw about the validity, reliability and broader utility of synchronous forms of telehealth physiotherapy assessments. Many studies used a small number of participants with a variety performing the assessment in simulated telehealth environments. There were a few studies, where telehealth assessments were conducted on a convenience sample of participants not necessarily reflective of the wider community or target group for the assessment type - for example, range of movement assessments performed on a healthy population and the MABC-2 performed on typically developing children. The methodological quality of the papers varied, with some papers not adequately describing the telehealth assessments completed or reporting limited information in their findings. The repeated measures design used for many of the validity and reliability studies included in this review has the potential to create a learning effect, with results also influenced by the irritability of the client's condition. The use of a randomised order of testing or simultaneous testing in most studies helped to counteract this.

The experience of clinicians undertaking the telehealth assessments varied, with reports of higher rates of intra-rater reliability for more experienced clinicians [61]. Levels of training and experience with telehealth systems also varied and should be considered when interpreting results for use in the clinical practice setting. Less experienced clinicians may not achieve the same level of validity and reliability outlined in studies within this review. Telehealth, however, can foster further professional development of less experienced clinicians by enabling more experienced and specialised clinicians to join physiotherapy assessments from anywhere using telehealth, provided they have a stable internet connection and an appropriate device. This 'peer learning' was 
observed in the utility study by Cottrell and colleagues [46], however further studies investigating the validity and reliability of this method of assessment would be beneficial across a broad range of physiotherapy practice areas. Whilst some studies utilised experienced clinicians, some of these were not experienced in the telehealth assessment process. Training in telehealth use is suggested to improve clinician competence, knowledge and acceptance, leading to better skills in using the technology and a higher likelihood of utilising it with clients when suitable [89]. This is important to consider when developing further studies that investigate the use of synchronous telehealth physiotherapy assessments and when using it in a clinical setting.

This systematic review only included studies investigating assessments conducted by a physiotherapist or supervised physiotherapy student. Studies involving assessments conducted by other health professionals, including occupational therapists and medical practitioners, that may also be conducted by physiotherapists were excluded.

Due to the large heterogeneity of the articles that were included in this review and the assortment of analysis used to derive their outcomes, a meta-analysis was not able to be undertaken and therefore, a traditional risk of bias assessment was not conducted. Instead, risk of bias was carefully considered through the critical appraisals performed. Given this was an exploratory study, several case studies/series were included in the results of this systematic review. We acknowledge case studies are considered a low level of evidence and caution needs to be used when interpreting their findings. Some practical information provided through the collection of these case studies can, however, be useful in clinical practice, for example the practicalities of completing a telehealth assessment, including the time it takes to conduct an assessment and the immediate responses of health services during the COVID-19 pandemic.

This systematic review only investigated the validity and reliability of assessments conducted via synchronous forms of telehealth. Asynchronous forms of telehealth such as the use of mobile apps, websites, monitoring devices (eg, smart watches, images and recorded video that are stored and forwarded on to the health care practitioner) are also used within the clinical practice setting. These areas of physiotherapy assessment via telehealth require further investigation and analysis to determine their effectiveness in clinical practice.

\section{CONCLUSION}

Many types of physiotherapy assessments performed using synchronous forms of telehealth appear to be valid and reliable when compared to face-to-face assessments, including assessments of range of movement, muscle strength and endurance, pain intensity, special orthopaedic tests of the shoulder and elbow, Berg Balance Scale (total score), TUG, timed stance test, 6MWT, steps in 360-degree turn, MABC-2 (within 3 points), step test, ABILHAND assessment, active straight leg raise, and circumferential measures of the upper limb. However, most of the research to date on use of telehealth for physiotherapy assessments has been conducted in the MSK field of physiotherapy. Given the recent shift towards this method of service delivery throughout the globe, more research is needed across all areas of physiotherapy. Evidence of validity, reliability and utility of assessments across other areas of physiotherapy practice is emerging but more research with larger sample sizes investigating synchronous telehealth physiotherapy assessments performed in real-world contexts, on readily available technologies and for a range of impairments, is required.

Acceptability of physiotherapy assessments across synchronous methods of telehealth is variable with most still preferring a face-to-face assessment if available. For those requiring telehealth services due to a lack of access to suitable services in their region, satisfaction is high. Telehealth has been vital in maintaining service provision throughout the COVID-19 pandemic however future use by physiotherapists must be decided with consideration given to utilisation of correct and accessible technology and contextual factors such as environmental variables, service access and individual needs and preferences. Telehealth has the potential to limit service access issues, however further research is required to determine which populations would benefit most.

\section{Contribution of the review}

This paper provides a comprehensive synthesis and review of the current research investigating the validity, reliability and utility of performing physiotherapy assessments using synchronous forms of telehealth across all areas of physiotherapy practice. The findings of this review are highly relevant to practitioners and researchers who are providing and/or conducting research in physiotherapy telehealth practice across the globe. 


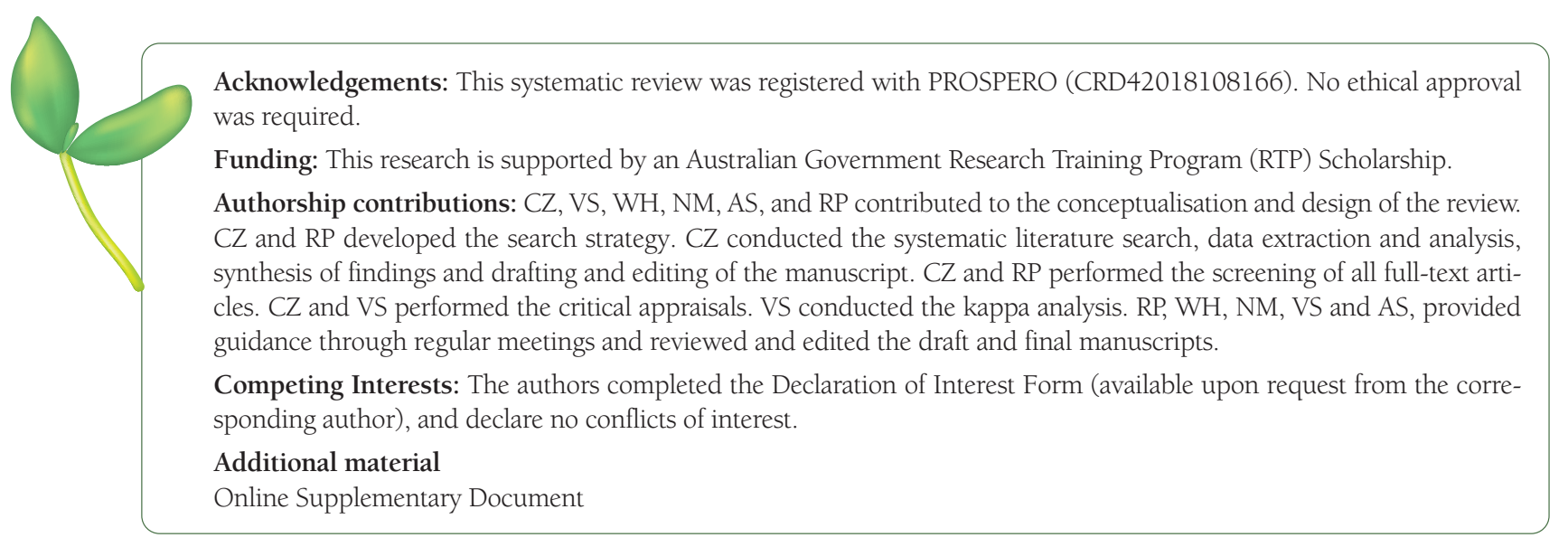

1 Deslauriers S, Déry J, Proulx K, Laliberté M, Desmeules F, Feldman DE, et al. Effects of waiting for outpatient physiotherapy services in persons with musculoskeletal disorders: a systematic review. Disabil Rehabil. 2021;43:611-20. Medline:31304824 doi:10.1080/09638288.2019.1639222

2 Dew A, Veitch C, Lincoln M, Brentnall J, Bulkeley K, Gallego G, et al. The need for new models for delivery of therapy intervention to people with a disability in rural and remote areas of Australia. J Intellect Dev Disabil. 2012;37:50-3. Medline:22211532 doi:10.3109/13668250.2011.644269

3 Adams R, Jones A, Lefmann S, Sheppard L. Towards understanding the availability of physiotherapy services in rural Australia. Rural Remote Health. 2016;16:3686. Medline:27289169 doi:10.22605/RRH3686

4 Russell T, Truter P, Blumke R, Richardson B. The diagnostic accuracy of telerehabilitation for nonarticular lower-limb musculoskeletal disorders. Telemed J E Health. 2010;16:585-94. Medline:20575726 doi:10.1089/tmj.2009.0163

5 Williams EN, McMeeken JM. Building capacity in the rural physiotherapy workforce: a paediatric training partnership. Rural Remote Health. 2014;14:2475. Medline:24655347 doi:10.22605/RRH2475

6 Orlando JF, Beard M, Kumar S. Systematic review of patient and caregivers' satisfaction with telehealth videoconferencing as a mode of service delivery in managing patients' health. PLoS One. 2019;14:e0221848. Medline:31469865 doi:10.1371/journal.pone.0221848

7 Beard M, Orlando JF, Kumar S. Overcoming the tyranny of distance: An audit of process and outcomes from a pilot telehealth spinal assessment clinic. J Telemed Telecare. 2017;23:733-9. Medline:27534822 doi:10.1177/1357633X16664851

8 World Health Organisation. Health Sector Strategies: Telehealth. 2021. Available: https://www.who.int/teams/environment-climate-change-and-health/air-quality-and-health/health-care-activities/strategies. Accessed: 8 March 2021.

9 Australian Government Department of Health. Telehealth. 2015. Available: https://wwwl.health.gov.au/internet/main/publishing.nsf/Content/e-health-telehealth. Accessed: 18 March 2021.

10 Cary MP Jr, Spencer M, Carroll A, Hand DH, Amis K, Karan E, et al. Benefits and challenges of delivering tele-rehabilitation services to rural veterans. Home Healthc Now. 2016;34:440-6. Medline:27580283 doi:10.1097/NHH.0000000000000441

11 Minghelli B, Soares A, Guerreiro A, Ribeiro A, Cabrita C, Vitoria C, et al. Physiotherapy services in the face of a pandemic. Rev Assoc Med Bras. 2020;66:491-7. Medline:32578784 doi:10.1590/1806-9282.66.4.491

12 Nulle J, Nelson VS. Video visits and access to care in pediatric rehabilitation therapies in the time of a pandemic. J Pediatr Rehabil Med. 2020;13:385-8. Medline:33104052 doi:10.3233/PRM-200759

13 Drouin JS, Chigbo NN, Newstead AH. Physiotherapist adaptations to cancer, HIV/AIDS, and hospice and palliative care in the COVID-19 Era: A global perspective paper. Rehabil Oncol. 2020;38:145-52. doi:10.1097/01.REO.0000000000000231

14 Cottrell MA, Russell TG. Telehealth for musculoskeletal physiotherapy. Musculoskelet Sci Pract. 2020;48:102193. Medline:32560876 doi:10.1016/j.msksp.2020.102193

15 MacDonald CW, Lonnemann E, Petersen SM, Rivett DA, Osmotherly PG, Brismée JM. COVID 19 and manual therapy: International lessons and perspectives on current and future clinical practice and education. J Man Manip Ther. 2020;28:134-45. Medline:32666912 doi:10.1080/10669817.2020.1782059

16 Australian Physiotherapy Association. Telehealth guidelines: Response to COVID-19. 2020. Available: https://australian.physio/sites/default/files/APA_Telehealth-Guidelines-COVID19_FA.PDF. Accessed: 18 March 2021.

17 NHS. Clinical guide for the management if remote consultations and remote working in secondary care during the coronavirus pandemic. 2020. Available: https://www.england.nhs.uk/coronavirus/wp-content/uploads/sites/52/2020/03/C0044-Specialty-Guide-Virtual-Working-and-Coronavirus-27-March-20.pdf. Accessed: 27 March 2021.

18 Abrashkin KA, Zhang J, Poku A. Acute, post-acute, and primary care utilization in a home-based primary care program during COVID-19. Gerontologist. 2021;61:78-85. Medline:33045054 doi:10.1093/geront/gnaal58

19 Mani S, Sharma S, Omar B, Paungmali A, Joseph L. Validity and reliability of Internet-based physiotherapy assessment for musculoskeletal disorders: a systematic review. J Telemed Telecare. 2017;23:379-91. Medline:27036879 doi:10.1177/1357633X16642369

20 Tecklin J. Pediatric Physical Therapy. 4th Edition ed. Philadelphia: Lippincott Williams \& Wilkins; 2008. 
21 Dario AB, Moreti Cabral A, Almeida L, Ferreira ML, Refshauge K, Simic M, et al. Effectiveness of telehealth-based interventions in the management of non-specific low back pain: A systematic review with meta-analysis. Spine J. 2017;17:1342-51. Medline:28412562 doi:10.1016/j.spinee.2017.04.008

22 Di Tella S, Pagliari C, Blasi V, Mendozzi L, Rovaris M, Baglio F. Integrated telerehabilitation approach in multiple sclerosis: A systematic review and meta-analysis. J Telemed Telecare. 2020;26:385-99. Medline:31132909 doi:10.1177/1357633X19850381

23 Cottrell MA, Galea OA, O'Leary SP, Hill AJ, Russell TG. Real-time telerehabilitation for the treatment of musculoskeletal conditions is effective and comparable to standard practice: A systematic review and meta-analysis. Clin Rehabil. 2017;31:625-38. Medline:27141087 doi:10.1177/0269215516645148

24 Russell TG, Jull GA, Wootton R. Can the Internet be used as a medium to evaluate knee angle? Man Ther. 2003;8:242-6. Medline:14559047 doi:10.1016/S1356-689X(03)00016-X

25 Grona SL, Bath B, Busch A, Rotter T, Trask C, Harrison E. Use of videoconferencing for physical therapy in people with musculoskeletal conditions: A systematic review. J Telemed Telecare. 2018;24:341-55. Medline:28403669 doi:10.1177/1357633X17700781

26 Block VA, Pitsch E, Tahir P, Cree BA, Allen DD, Gelfand JM. Remote physical activity monitoring in neurological disease: A systematic review. PLoS One. 2016;11:e0154335. Medline:27124611 doi:10.1371/journal.pone.0154335

27 Godinho C, Domingos J, Cunha G, Santos AT, Fernandes RM, Abreu D, et al. A systematic review of the characteristics and validity of monitoring technologies to assess Parkinson's disease. J Neuroeng Rehabil. 2016;13:24. Medline:26969628 doi:10.1186/s12984-016-0136-7

28 Liu L, Stroulia E, Nikolaidis I, Miguel-Cruz A, Rios Rincon A. Smart homes and home health monitoring technologies for older adults: A systematic review. Int J Med Inform. 2016;91:44-59. Medline:27185508 doi:10.1016/j.ijmedinf.2016.04.007

29 The EndNote Team. EndNote. EndNote X9 ed. Philadelphia, PA: Clarivate; 2013.

30 Page MJ, McKenzie JE, Bossuyt PM, Boutron I, Hoffmann TC, Mulrow CD, et al. The PRISMA 2020 statement: An updated guideline for reporting systematic reviews. BMJ. 2021;372:n71. Medline:33782057 doi:10.1136/bmj.n71

31 Brink Y, Louw QA. Clinical instruments: Reliability and validity critical appraisal. J Eval Clin Pract. 2012;18:1126-32. Medline:21689217 doi:10.1111/j.1365-2753.2011.01707.x

32 Barrett E, McCreesh K, Lewis J. Reliability and validity of non-radiographic methods of thoracic kyphosis measurement: A systematic review. Man Ther. 2014;19:10-7. Medline:24246907 doi:10.1016/j.math.2013.09.003

33 Adhia DB, Bussey MD, Ribeiro DC, Tumilty S, Milosavljevic S. Validity and reliability of palpation-digitization for non-invasive kinematic measurement: A systematic review. Man Ther. 2013;18:26-34. Medline:22784802 doi:10.1016/j.math.2012.06.004

34 Farahmand B, Ebrahimi Takamjani E, Yazdi HR, Saeedi H, Kamali M, Bagherzadeh Cham M. A systematic review on the validity and reliability of tape measurement method in leg length discrepancy. Med J Islam Repub Iran. 2019;33:46. Medline:31456970 doi:10.47176/mjiri.33.46

35 Pivotto LR, Navarro I, Candotti CT. Radiography and photogrammetry-based methods of assessing cervical spine posture in the sagittal plane: A systematic review with meta-analysis. Gait Posture. 2021;84:357-67. Medline:33465736 doi:10.1016/j. gaitpost.2020.12.033

36 Hong QN, Pluye P, Fabregues S, Bartlett G, Boardman F, Cargo M, et al. Mixed Methods Appraisal Tool (MMAT) version 2018. 2018.

37 Hong QN, Gonzalez-Reyes A, Pluye P. Improving the usefulness of a tool for appraising the quality of qualitative, quantitative and mixed methods studies: The Mixed Methods Appraisal Tool (MMAT). J Eval Clin Pract. 2018;24:459-67. Medline:29464873 doi:10.1111/jep. 12884

38 Hong QN. Reporting the results of the MMAT (version 2018). Available: https://www.who.int/teams/environment-climate-change-and-health/air-quality-and-health/health-care-activities/strategies. Accessed: 8 March 2021.

39 Cohen J. A coefficient of agreement for nominal scales. Educ Psychol Meas. 1960;20:37-46. doi:10.1177/001316446002000104

40 NHMRC. NHMRC levels of evidence and grades for recommendations for developers of guidelines. Australian Government; 2009.

41 Avelino PR, Menezes KKP, Nascimento LR, Tenório RA, Cândido GN, Christovão IS, et al. Validation of the telephone-based application of the ABILHAND for assessment of manual ability after stroke. J Neurol Phys Ther. 2020;44:256-60. Medline:32815891 doi:10.1097/NPT.0000000000000326

42 Boggs R, Frappa N, Ross M, Tall M. Telehealth and physical therapy clinical decision making in a patient with a falcine meningioma. Int J Telerehabil. 2020;12:63-8. Medline:32983369 doi:10.5195/ijt.2020.6302

43 Cabana F, Boissy P, Tousignant M, Moffet H, Corriveau H, Dumais R. Interrater agreement between telerehabilitation and faceto-face clinical outcome measurements for total knee arthroplasty. Telemed J E Health. 2010;16:293-8. Medline:20406116 doi:10.1089/tmj.2009.0106

44 Conlan L, Thompson J, Fary R. An exploration of the efficacy of telehealth in the assessment and management of stress urinary incontinence among women in rural locations. ANZCJ. 2016;22:58-64.

45 Cottrell MA, O'Leary SP, Swete-Kelly P, Elwell B, Hess S, Litchfield MA, et al. Agreement between telehealth and in-person assessment of patients with chronic musculoskeletal conditions presenting to an advanced-practice physiotherapy screening clinic. Musculoskelet Sci Pract. 2018;38:99-105. Medline:30366292 doi:10.1016/j.msksp.2018.09.014

46 Cottrell M, Judd P, Comans T, Easton P, Chang AT. Comparing fly-in fly-out and telehealth models for delivering advanced-practice physiotherapy services in regional Queensland: An audit of outcomes and costs. J Telemed Telecare. 2021;27:32-8. Medline:31280639 doi:10.1177/1357633X19858036

47 Cox NS, Alison JA, Button BM, Wilson JW, Holland AE. Assessing exercise capacity using telehealth: A feasibility study in adults with cystic fibrosis. Respir Care. 2013;58:286-90. Medline:22711058 
48 Demmelmaier I, Denison E, Lindberg P, Asenlof P. Physiotherapists' telephone consultations regarding back pain: A method to analyze screening of risk factors. Physiother Theory Pract. 2010;26:468-75. Medline:20649497 doi:10.3109/09593980903433938

49 Eannucci EF, Hazel K, Grundstein MJ, Nguyen JT, Gallegro J. Patient satisfaction for telehealth physical therapy services was comparable to that of in-person services during the COVID-19 pandemic. HSS J. 2020;16(Suppl 1):1-7. Medline:33100933 doi:10.1007/s11420-020-09800-5

50 Exum E, Hull BL, Lee ACW, Gumieny A, Villarreal C, Longnecker D. Applying telehealth technologies and strategies to provide acute care consultation and treatment of patients with confirmed or possible COVID-19. J Acute Care Phys Ther. 2020;11:10312. Medline:34192091 doi:10.1097/JAT.0000000000000143

51 Funderskov KF, Boe Danbjørg D, Jess M, Munk L, Olsen Zwisler AD, Dieperink KB. Telemedicine in specialised palliative care: Healthcare professionals' and their perspectives on video consultations: A qualitative study. J Clin Nurs. 2019;28:3966-76. Medline:31328336 doi:10.1111/jocn.15004

52 Galiano-Castillo N, Ariza-García A, Cantarero-Villanueva I, Fernández-Lao C, Sánchez-Salado C, Arroyo-Morales M. Agreement between telerehabilitation involving caregivers and face-to-face clinical assessment of lymphedema in breast cancer survivors. Support Care Cancer. 2014;22:253-8. Medline:24043290 doi:10.1007/s00520-013-1971-8

53 Lovo Grona S, Bath B, Bustamante L, Mendez I. Case report: Using a remote presence robot to improve access to physical therapy for people with chronic back disorders in an underserved community. Physiother Can. 2017;69:14-9. Medline:28154440 doi:10.3138/ptc.2015-77

54 Harland N, Blacklidge B. Physiotherapists and general practitioners attitudes towards 'Physio Direct' phone based musculoskeletal physiotherapy services: a national survey. Physiotherapy. 2017;103:174-9. Medline:27913062 doi:10.1016/j.physio.2016.09.002

55 Hollinghurst S, Coast J, Busby J, Bishop A, Foster NE, Franchini A, et al. A pragmatic randomised controlled trial of 'PhysioDirect' telephone assessment and advice services for patients with musculoskeletal problems: Economic evaluation. BMJ Open. 2013;3:e003406. Medline:24091423 doi:10.1136/bmjopen-2013-003406

56 Hwang R, Mandrusiak A, Morris NR, Peters R, Korczyk D, Russell T. Assessing functional exercise capacity using telehealth: Is it valid and reliable in patients with chronic heart failure? J Telemed Telecare. 2017;23:225-32. Medline:26915366 doi:10.1177/1357633X16634258

57 Kinder J, Davenport T, Chong W, Lee A. Telerehabilitation for treating pelvic floor dysfunction: A case series of 3 patients' experiences. J Womens Health Phys Therap. 2019;43:44-50. doi:10.1097/JWH.0000000000000120

58 Lade H, McKenzie S, Steele L, Russell TG. Validity and reliability of the assessment and diagnosis of musculoskeletal elbow disorders using telerehabilitation. J Telemed Telecare. 2012;18:413-8. Medline:23086982 doi:10.1258/jtt.2012.120501

59 Lovo S, Harrison L, O'Connell ME, Trask C, Bath B. Experience of patients and practitioners with a team and technology approach to chronic back disorder management. J Multidiscip Healthc. 2019;12:855-69. Medline:31695402 doi:10.2147/ JMDH.S208888

60 Mani S, Sharma S, Singh DK. Concurrent validity and reliability of telerehabilitation-based physiotherapy assessment of cervical spine in adults with non-specific neck pain. J Telemed Telecare. 2021:88-97. Medline:31272309 doi:10.1177/1357633X19861802

61 Mehta SP, Kendall KM, Reasor CM. Virtual assessments of knee and wrist joint range motion have comparable reliability with face-to-face assessments. Musculoskeletal Care. 2021;19:208-16. Medline:33107154 doi:10.1002/msc.1525

62 Mukaino M, Tatemoto T, Kumazawa N, Tanabe S, Katoh M, Saitoh E, et al. An affordable, user-friendly telerehabilitation system assembled using existing technologies for individuals isolated with COVID-19: Development and feasibility study. JMIR Rehabil Assist Technol. 2020;7:e24960. Medline:33279877 doi:10.2196/24960

63 Nicola K, Waugh J, Charles E, Russell T. The feasibility and concurrent validity of performing the Movement Assessment Battery for Children - 2nd Edition via telerehabilitation technology. Res Dev Disabil. 2018;77:40-8. Medline:29656273 doi:10.1016/j ridd.2018.04.001

64 O'Donovan M, Buckley C, Benson J, Roche S, McGowan M, Parkinson L, et al. Telehealth for delivery of haemophilia comprehensive care during the COVID-19 pandemic. Haemophilia. 2020;26:984-90. Medline:32997849 doi:10.1111/hae.14156

65 Palacín-Marín F, Esteban-Moreno B, Olea N, Herrera-Viedma E, Arroyo-Morales M. Agreement between telerehabilitation and face-to-face clinical outcome assessments for low back pain in primary care. Spine. 2013;38:947-52. Medline:23238489 doi:10.1097/BRS.0b013e318281a36c

66 Pearson J, Richardson J, Calnan M, Salisbury C, Foster N. Acceptability to patients of PhysioDirect telephone advice and treatment services: A qualitative investigation. Physiother Res Int. 2013;18:63. doi:10.1002/pri.1519

67 Peterson S, Kuntz C, Roush J. Use of a modified treatment-based classification system for subgrouping patients with low back pain: Agreement between telerehabilitation and face-to-face assessments. Physiother Theory Pract. 2019;35:1078-86. Medline:29723124 doi:10.1080/09593985.2018.1470210

68 Prada V, Bellone E, Schenone A, Grandis M. The suspected SARS-Cov-2 infection in a Charcot-Marie-Tooth patient undergoing postsurgical rehabilitation: The value of telerehabilitation for evaluation and continuing treatment. Int J Rehabil Res. 2020;43:285-6. Medline:32317558 doi:10.1097/MRR.0000000000000418

69 Richardson BR, Truter P, Blumke R, Russell TG. Physiotherapy assessment and diagnosis of musculoskeletal disorders of the knee via telerehabilitation. J Telemed Telecare. 2017;23:88-95. Medline:26985005 doi:10.1177/1357633X15627237

70 Russell TG. Internet-based physical assessment of people with Parkinson disease is accurate and reliable: A pilot study. J Rehabil Res Dev. 2013;50:643-50. Medline:24013912 doi:10.1682/JRRD.2012.08.0148

71 Russell TG, Blumke R, Richardson B, Truter P. Telerehabilitation mediated physiotherapy assessment of ankle disorders. Physiother Res Int. 2010;15:167-75. Medline:20812313 doi:10.1002/pri.471 
72 Salisbury C, Montgomery AA, Hollinghurst S, Hopper C, Bishop A, Franchini A, et al. Effectiveness of PhysioDirect telephone assessment and advice services for patients with musculoskeletal problems: Pragmatic randomised controlled trial. BMJ. 2013;346:f43. Medline:23360891 doi:10.1136/bmj.f43

73 Steele L, Lade H, McKenzie S, Russell TG. Assessment and diagnosis of musculoskeletal shoulder disorders over the internet. Int J Telemed Appl. 2012;2012:945745. Medline:23193395 doi:10.1155/2012/945745

74 Truter P, Russell T, Fary R. The validity of physical therapy assessment of low back pain via telerehabilitation in a clinical setting. Telemed J E Health. 2014;20:161-7. Medline:24283249 doi:10.1089/tmj.2013.0088

75 Turner A. Case studies in physical therapy: Transitioning a "hands-on" approach into a virtual platform. Int J Telerehabil. 2018;10:37-50. Medline:30147842 doi:10.5195/ijt.2018.6253

76 Wood J, Mulrennan S, Hill K, Cecins N, Morey S, Jenkins S. Telehealth clinics increase access to care for adults with cystic fibrosis living in rural and remote Western Australia. J Telemed Telecare. 2017;23:673-9. Medline:27444187 doi:10.1177/1357633X16660646

77 Wootton SL, King M, Alison JA, Mahadev S, Chan ASL. COVID-19 rehabilitation delivered via a telehealth pulmonary rehabilitation model: A case series. Respirol Case Rep. 2020;8:e00669. Medline:33042547 doi:10.1002/rcr2.669

78 Brooke J. SUS - A quick and dirty usability scale. Digital Equipment Corporation; 1996. p. 189-94.

79 Fatudimu MB. Influence of the environment on performance of gross motor function in children with cerebral palsy. J Pediatr Rehabil Med. 2012;5:181-6. Medline:23023250 doi:10.3233/PRM-2012-0210

80 The Dutch National Institute for Public Health and the Environment. Risk groups and COVID-19. 2021. Available: https:// www.rivm.nl/en/coronavirus-covid-19/risk-groups. Accessed: 11 May 2021.

81 Kruse CS, Krowski N, Rodriguez B, Tran L, Vela J, Brooks M. Telehealth and patient satisfaction: A systematic review and narrative analysis. BMJ Open. 2017;7:e016242. Medline:28775188 doi:10.1136/bmjopen-2017-016242

82 Kesharwani A. (How) digital natives adopt a new technology differently than digital immigrants? A longitudinal study. Inf Mang. 2020;57:103170. doi:10.1016/j.im.2019.103170

83 Pollard JS, Karimi KA, Ficcaglia MB. Ethical considerations in the design and implementation of a telehealth service delivery model. Behavior Analysis: Research and Practice. 2017;17:298-311. doi:10.1037/bar0000053

84 Rinaldi G, Hijazi A, Haghparast-Bidgoli H. Cost and cost-effectiveness of mHealth interventions for the prevention and control of type 2 diabetes mellitus: A systematic review. Diabetes Res Clin Pract. 2020;162:108084. Medline:32061819 doi:10.1016/j. diabres.2020.108084

85 Avidor D, Loewenstein A, Waisbourd M, Nutman A. Cost-effectiveness of diabetic retinopathy screening programs using telemedicine: a systematic review. Cost Eff Resour Alloc. 2020;18:16. Medline:32280309 doi:10.1186/s12962-020-00211-1

86 Naslund JA, Mitchell LM, Joshi U, Nagda D, Lu C. Economic evaluation and costs of telepsychiatry programmes: A systematic review. J Telemed Telecare. 2020:1357633X20938919. Medline:32746762 doi:10.1177/1357633X20938919

87 Norris M, Wainwright E. Learning professional touch: An exploration of pre-registration Physiotherapy students' experiences. Physiother Theory Pract. 2020:1-11. Medline:32053416 doi:10.1080/09593985.2020.1725944

88 Roy JS, MacDermid JC, Woodhouse LJ. Measuring shoulder function: A systematic review of four questionnaires. Arthritis Rheum. 2009;61:623-32. Medline:19405008 doi:10.1002/art.24396

89 McClellan MJ, Florell D, Palmer J, Kidder C. Clinician telehealth attitudes in a rural community mental health center setting. Rural Mental Health. 2020;44:62-73. doi:10.1037/rmh0000127 